$(200)$

R290

70.143

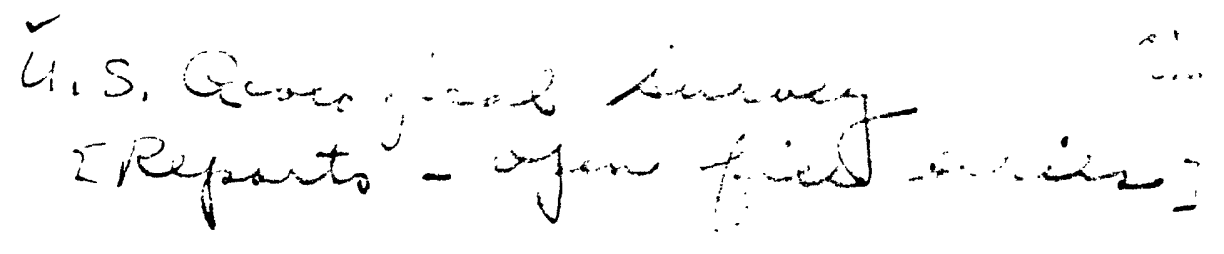

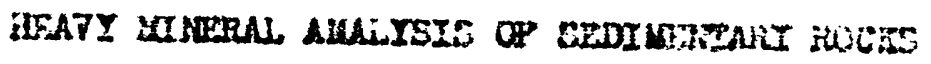

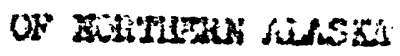

nowers n. pouss, 1921 -

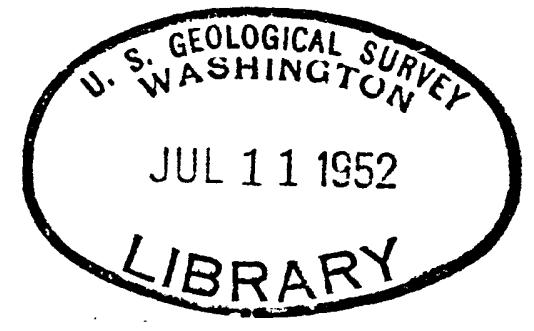

Lis: 2052

$52-102$ 
Rlobrat............................. 2

Introtiction ............................ 2

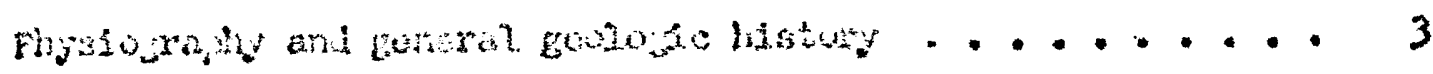

stratigaty ....................... 5

binted ofurates ....................... 6

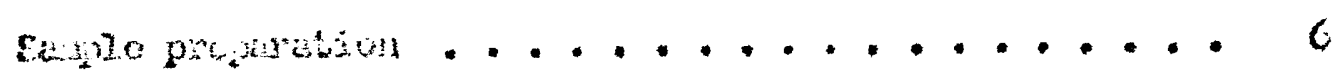

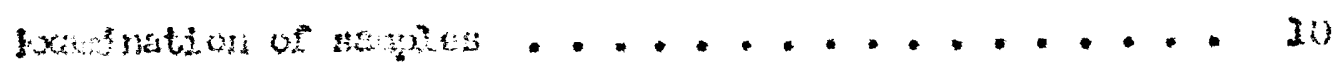

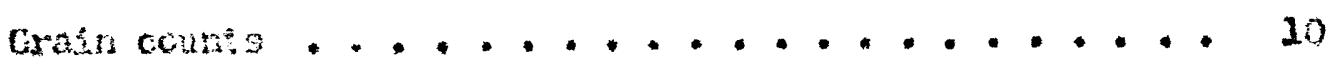

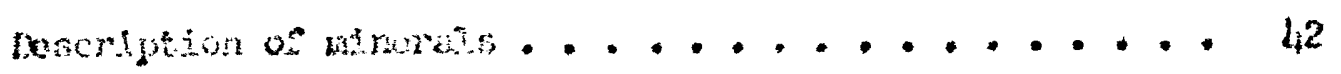

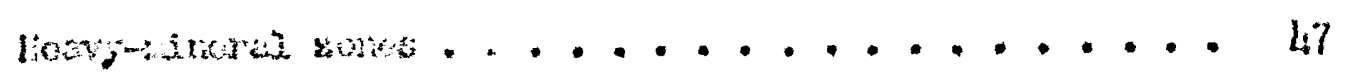

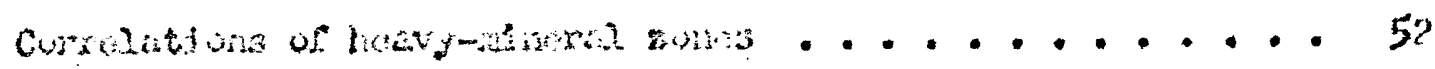

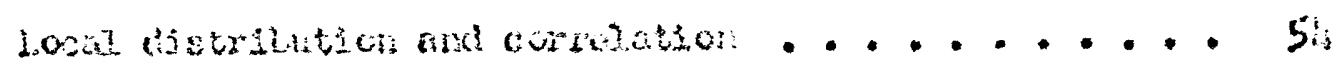

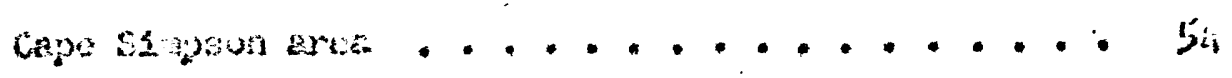

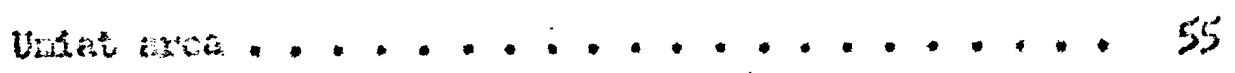

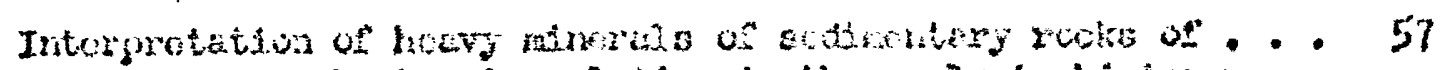

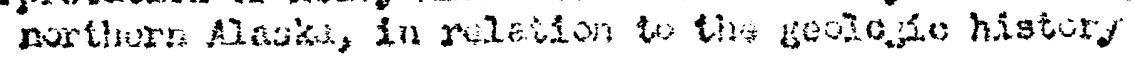

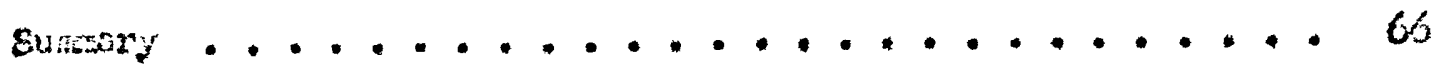

\section{Mustrutsuis and tableck}

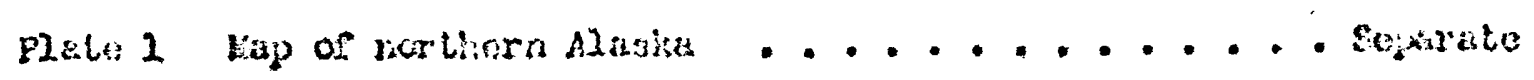

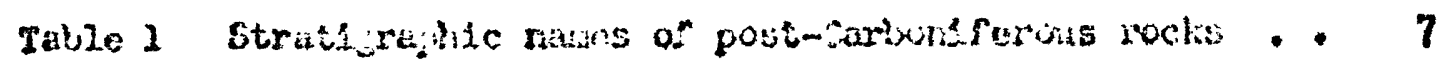

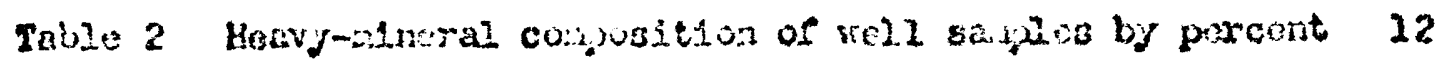

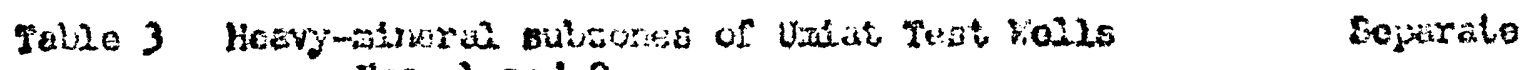
Mos. 1 and 2 
II? uotation and hables (contd)

$\operatorname{lag}$

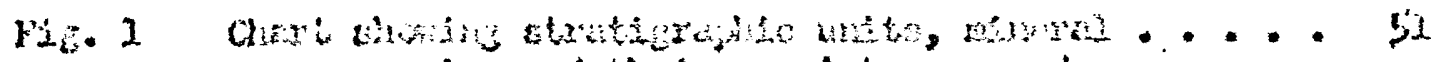

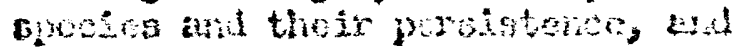

hectymand soment

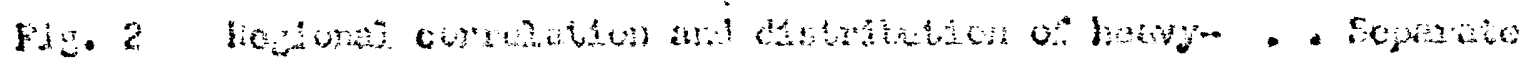

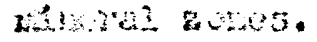

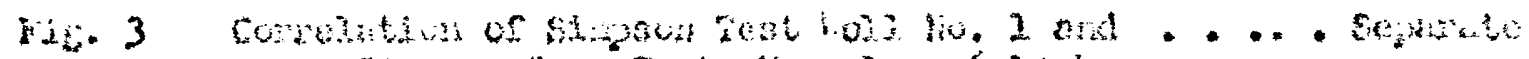

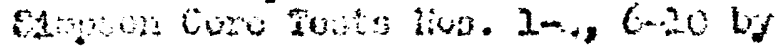

hity-itherat xwas.

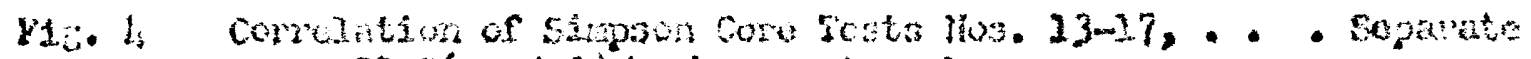

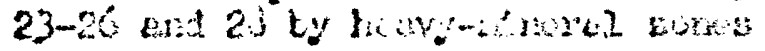

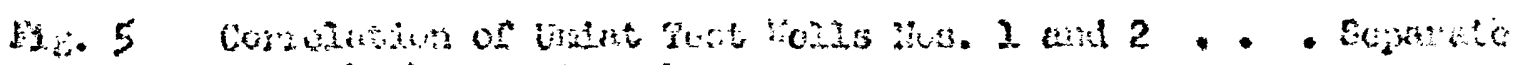

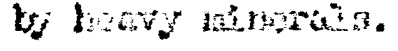




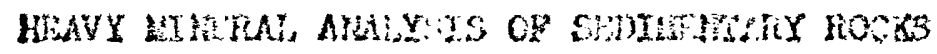

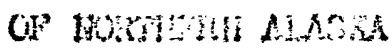

$1 y$

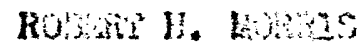

\section{A. 20101}

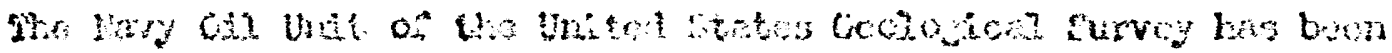

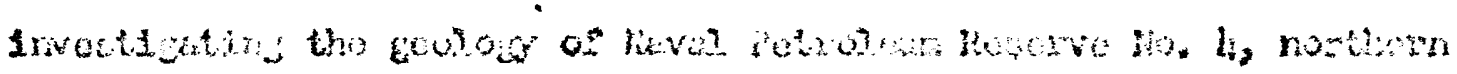

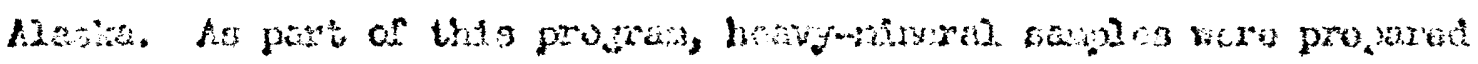

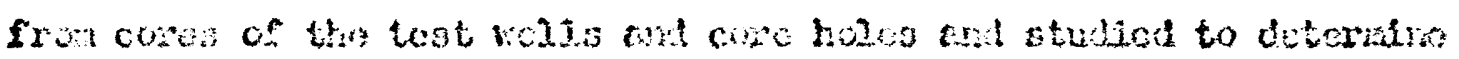

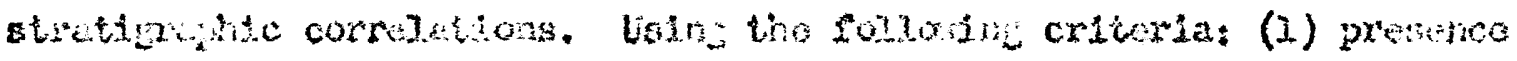

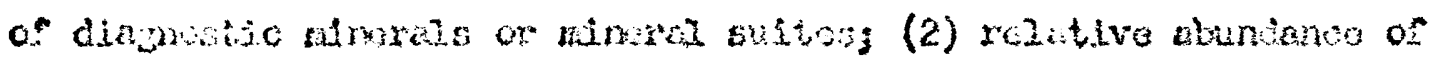

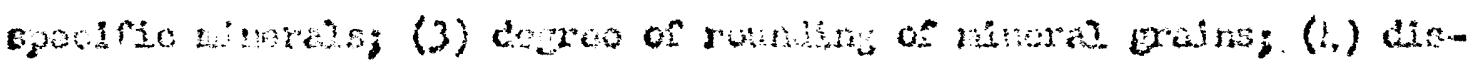

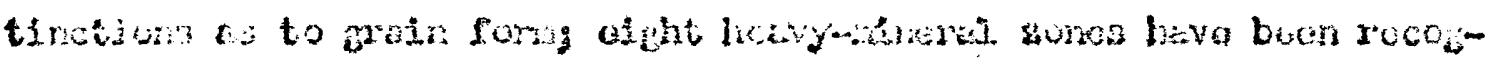

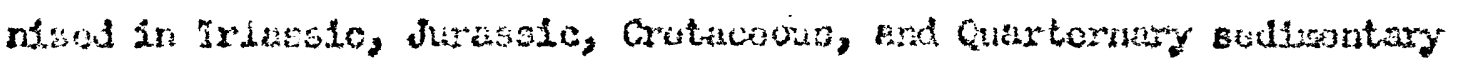

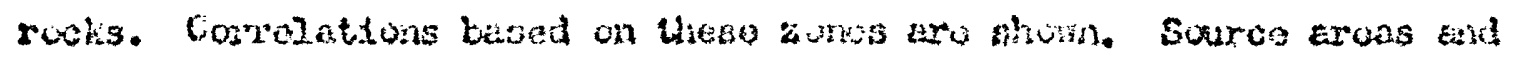

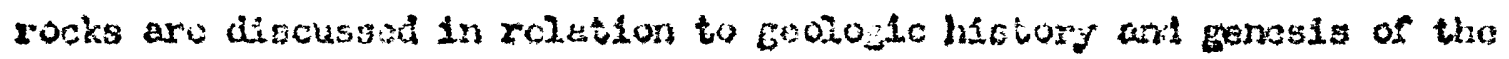

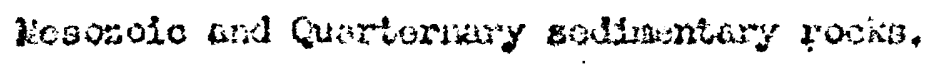




\section{IHRODUReV}

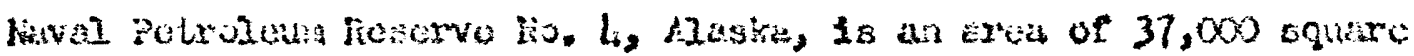

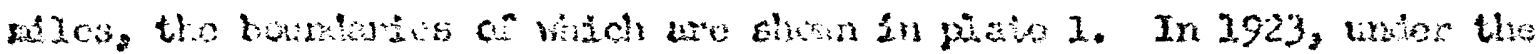

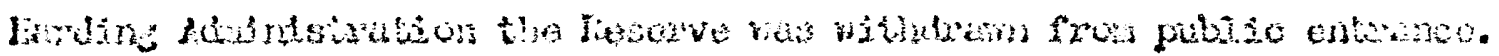

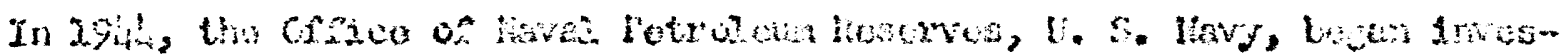

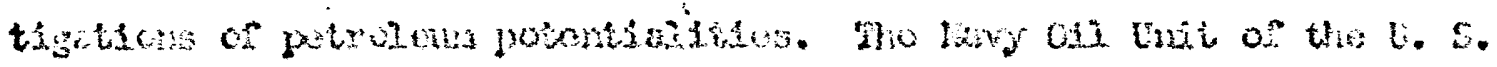

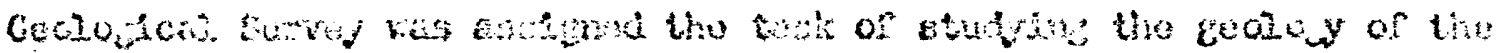

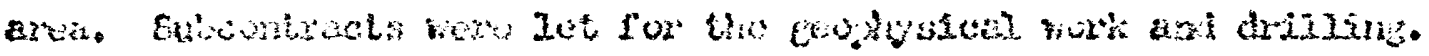

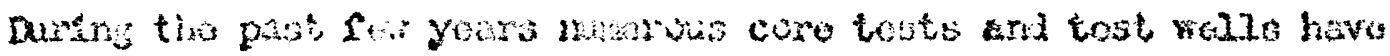

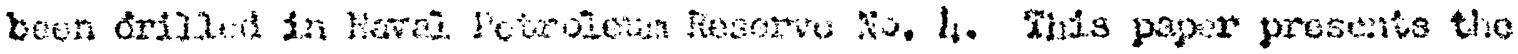

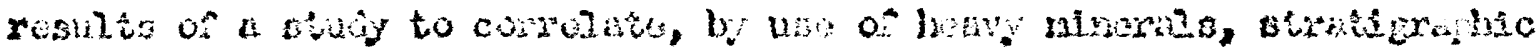

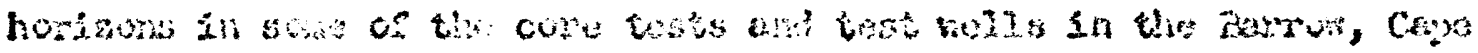

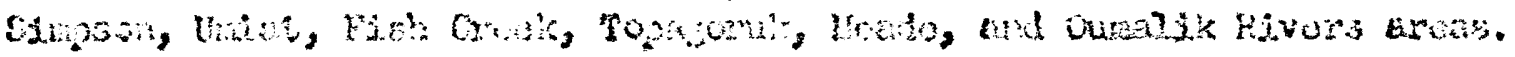

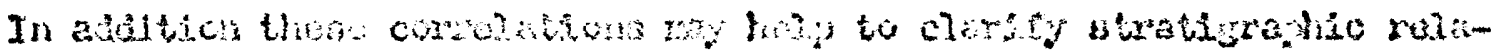

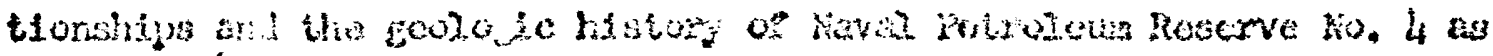
a whole.

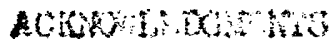

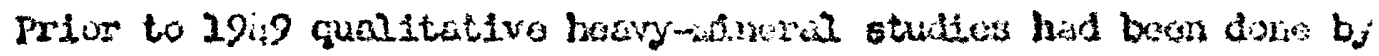

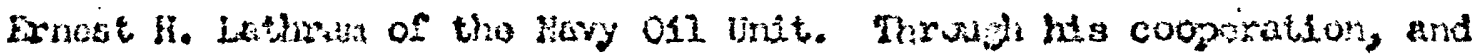

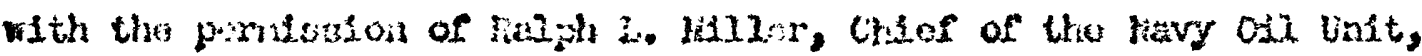

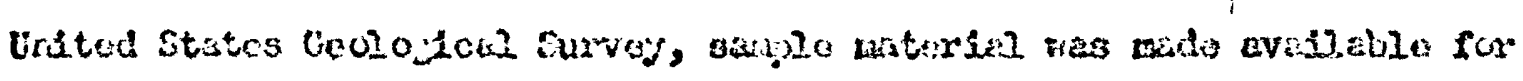

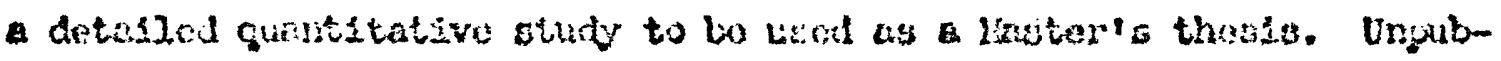

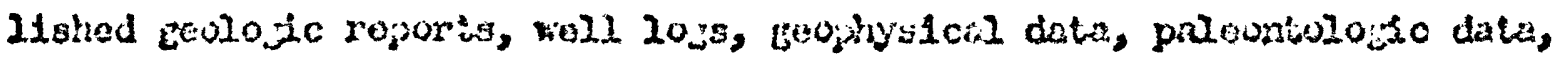
ard all portinint inforisition were mide avaliable to the authur. Gratenl epproclation 13 axtasded to 138s H. H. Eckstuin, United Statos Cealopical survey, for editins the manueript. 


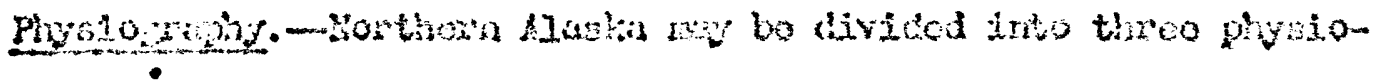

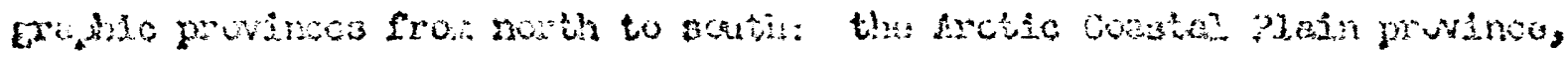

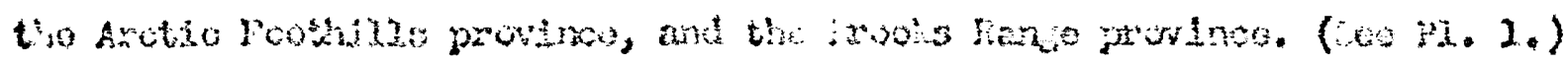

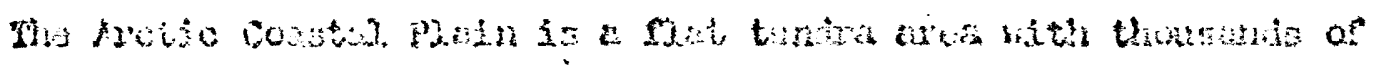

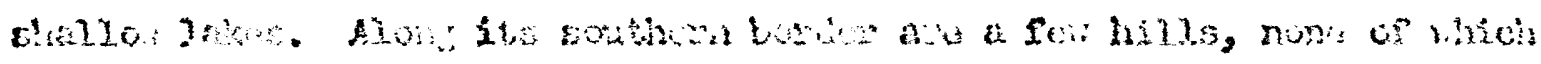

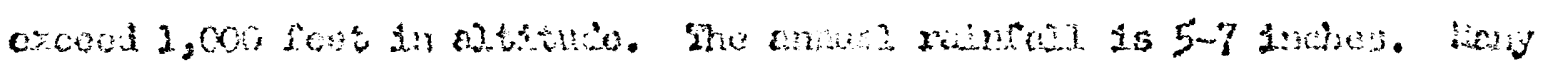

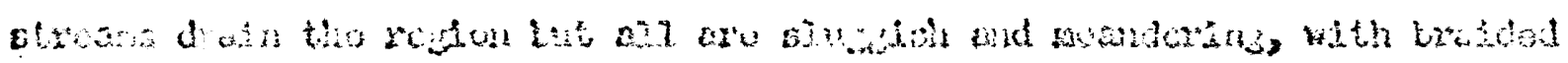

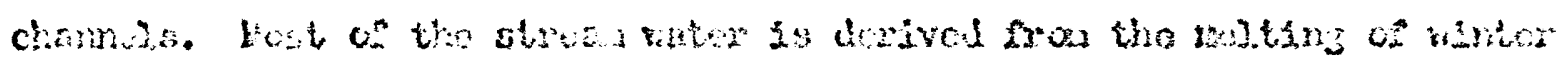
Envine.

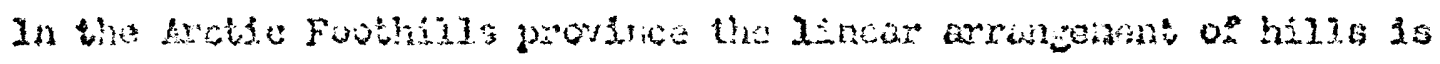

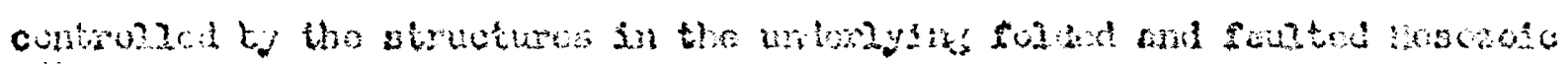

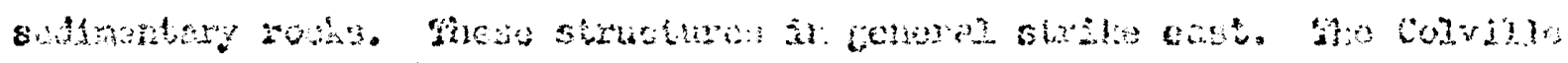

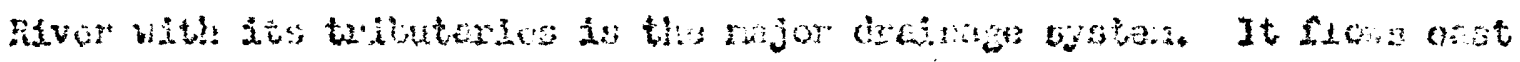

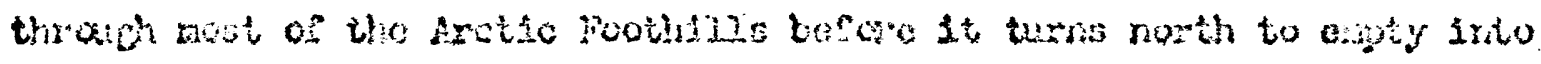

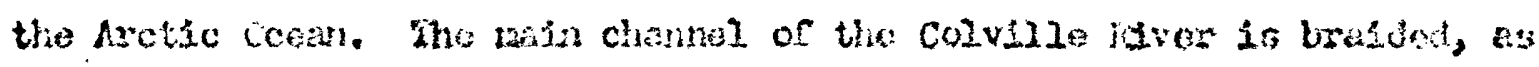

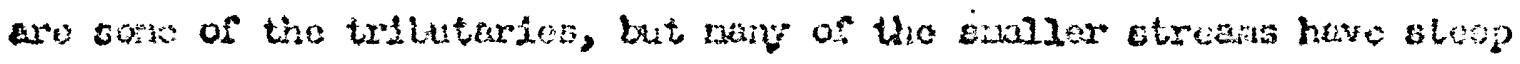

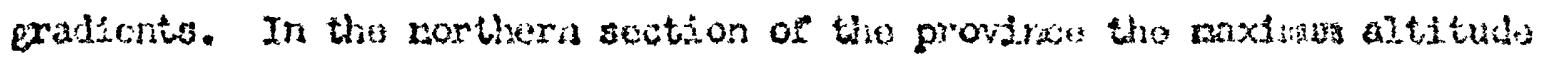

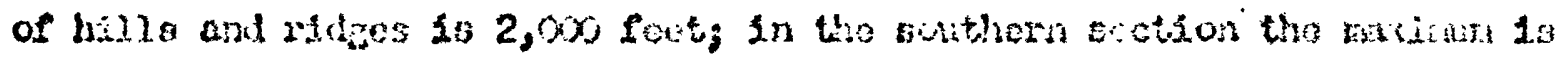
nore than 3,000 feet.

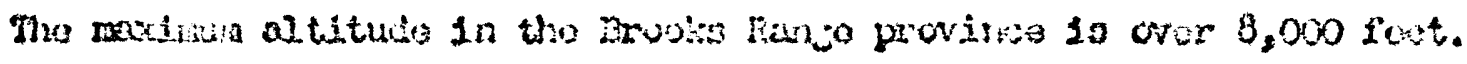

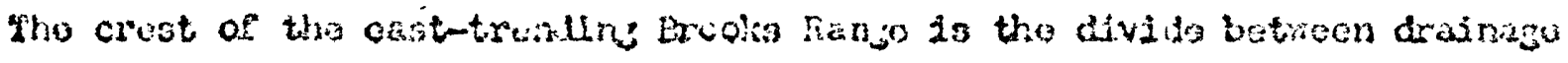

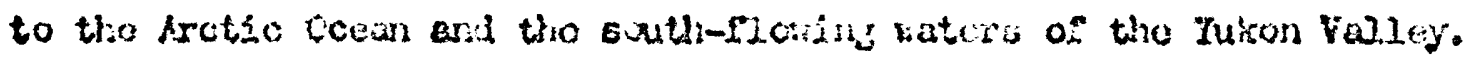


Hajor folds eral Jarge turust faults with rustuttant disjuacosonts to tho

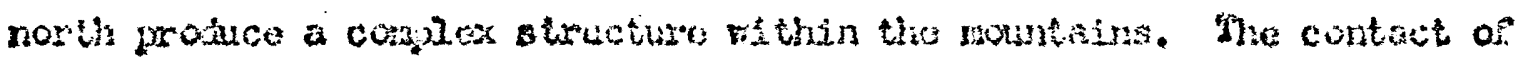

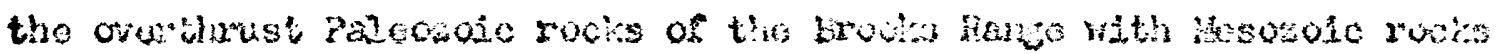

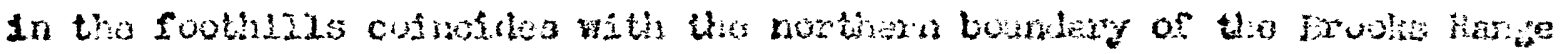

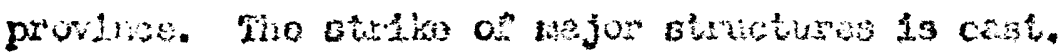

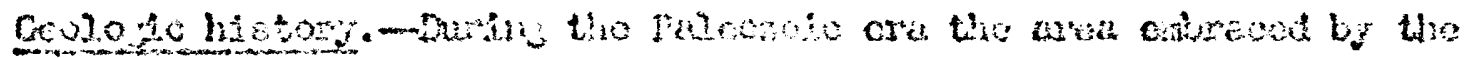

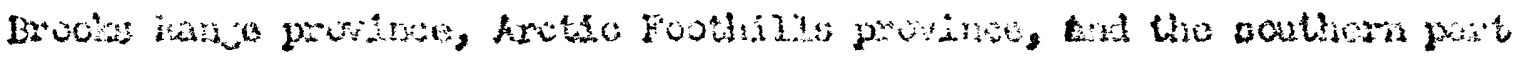

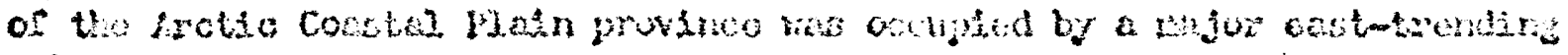

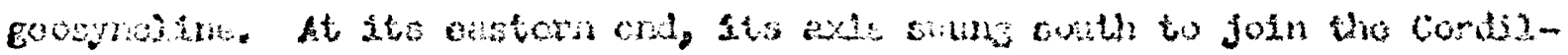

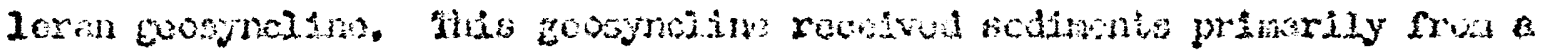

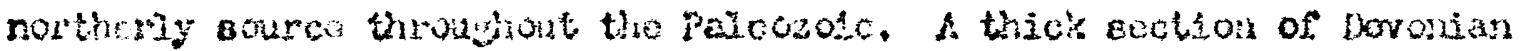

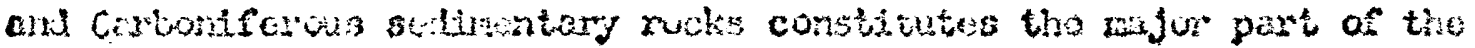
Drowat bus?

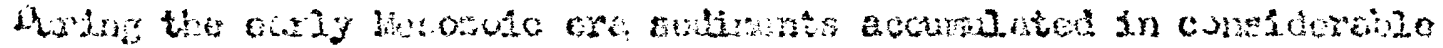

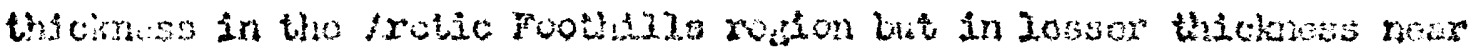

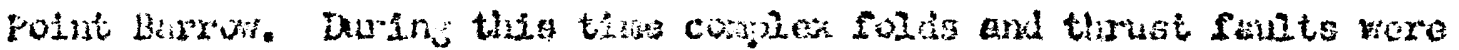

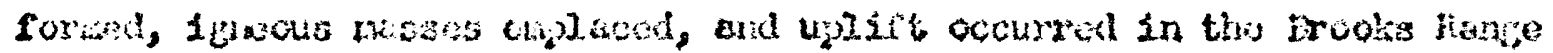

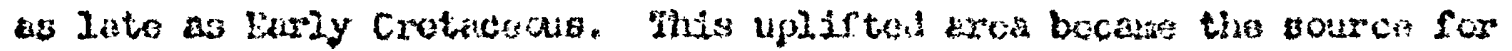

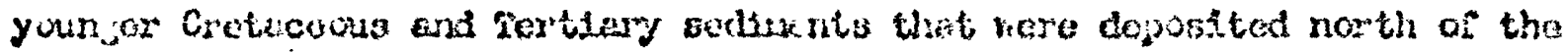

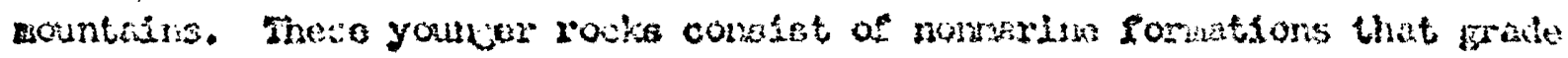
northmad into merin factos in tho constal plein rogion.

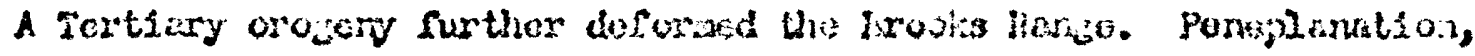

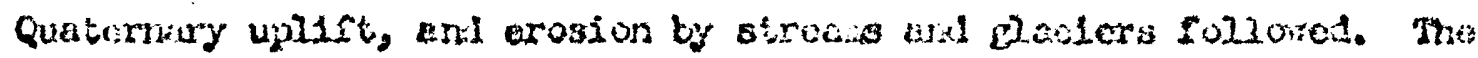
Erooks liange ras the site of alpinu clacione during tho plestotocene, 
Eheruas tho northera part of tho Arctic Foothitin and tha Arctic Coastial

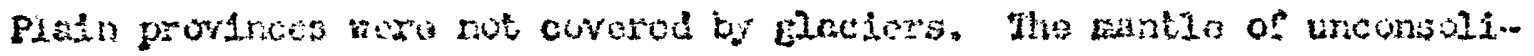

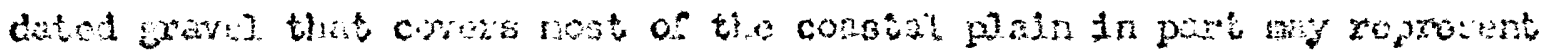

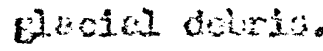

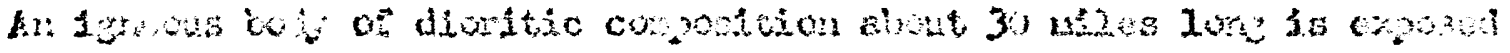

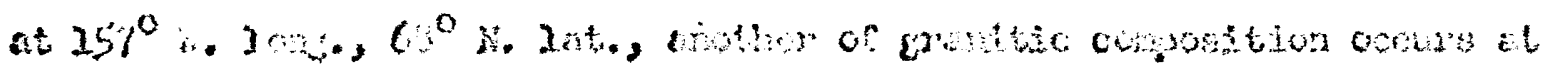

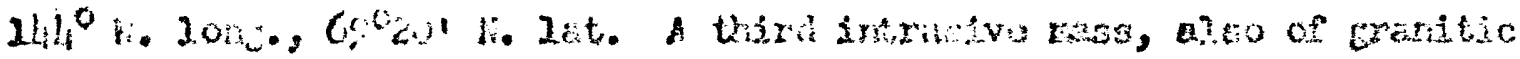

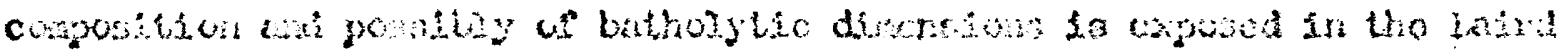

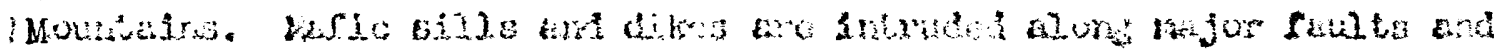

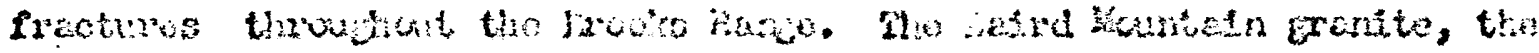

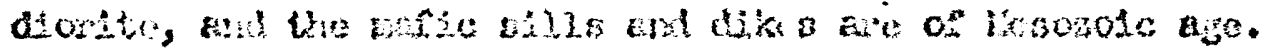

\section{Stamblath}

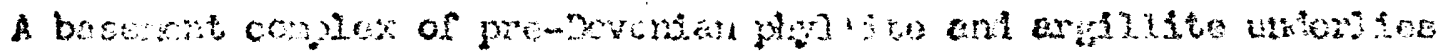

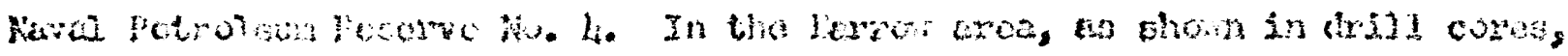

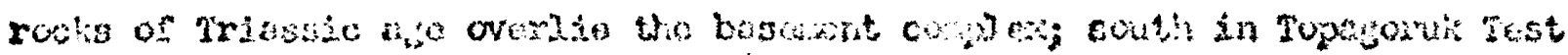

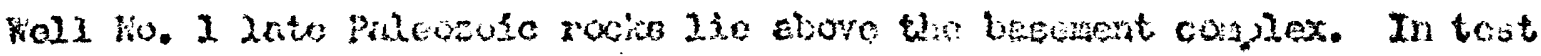

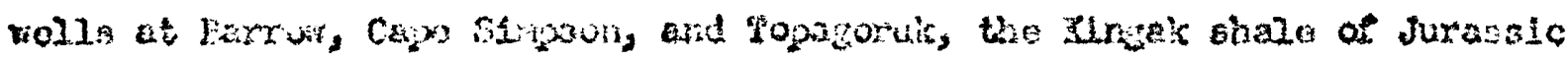

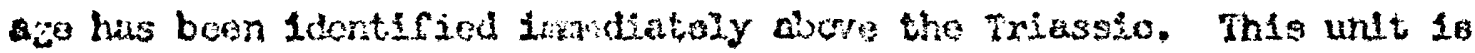

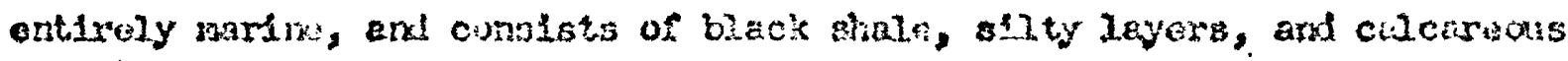
irongtone linuse.

Unconformbly abovo the Jurasalc rucks axo crutecedis intertonsing

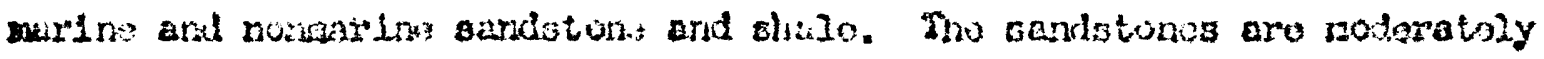

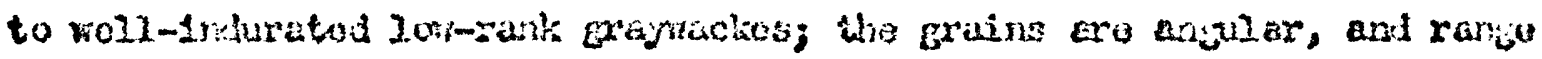

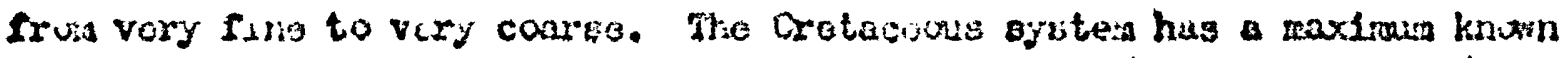
Wi1cienoss of abut 23,000 fect 60 willes south of Mah Crook Test Fell tio. 1. It Point Barror the oyatea io about 2,000 reot thick. 
Fosting on the eroded's rece of the cretscouts rocks is tho Gubik forintion of pleistocero age. Tho Cubik fondion ranges in thichess

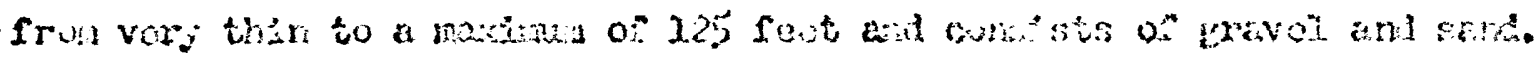

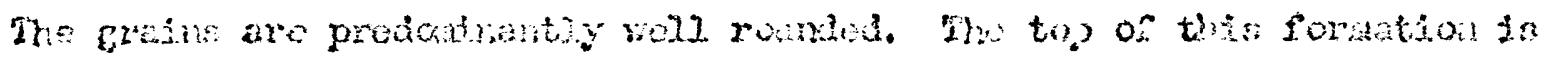

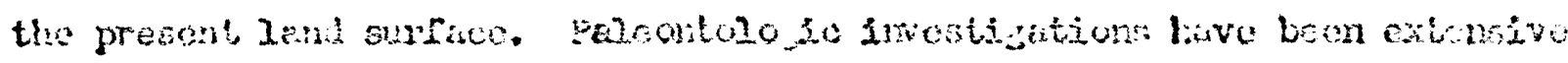

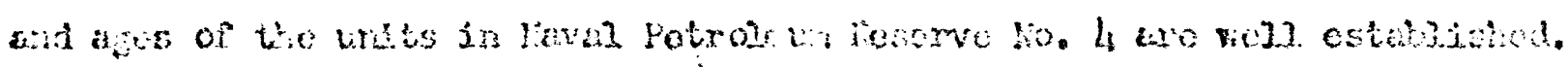

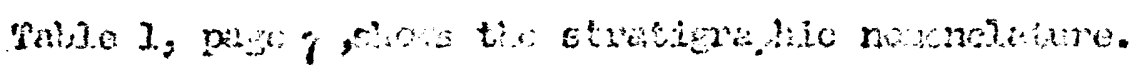

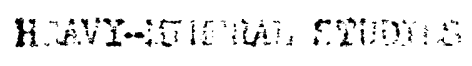

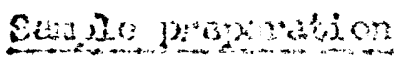

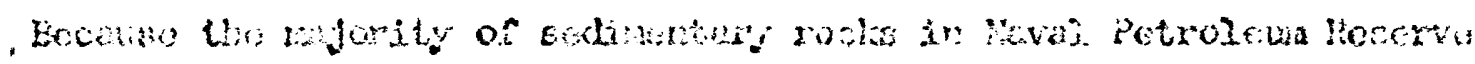

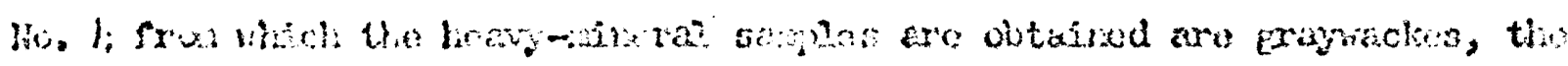

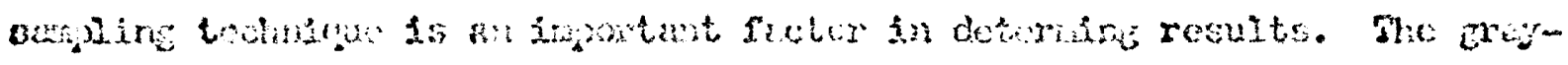

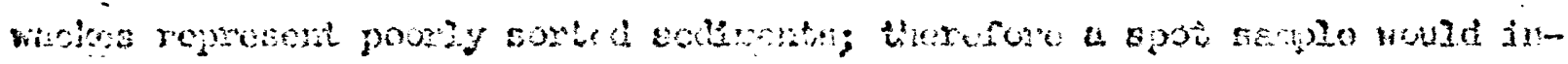

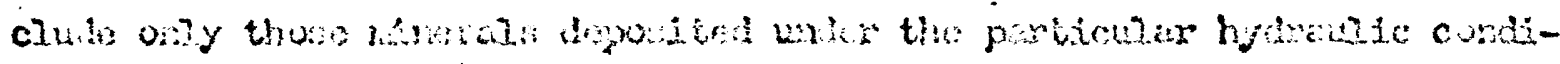

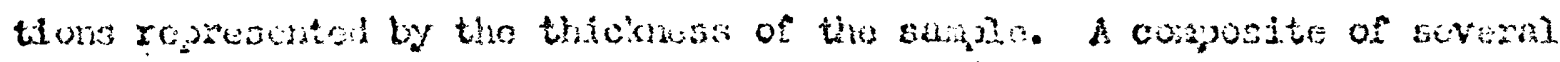

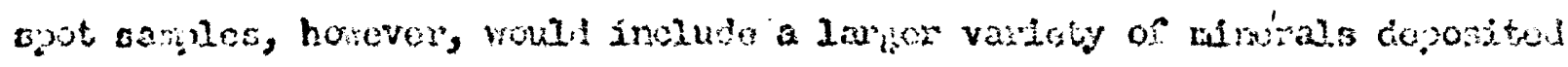

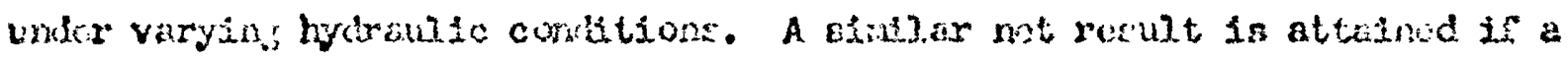

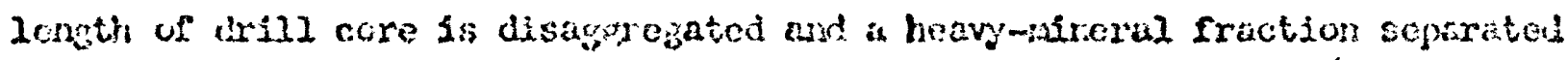

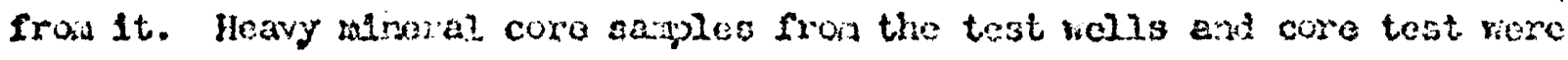
therefore propered in the followini manas. A 10woot section of sindstone was cored; fron this about 6 feot of cord hes the averais recovery sevcra? Buall anctions of this 6-foot coro row taken, in affest a canporits of the

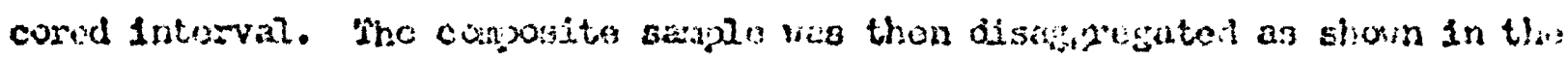
Ilor elscet on pasize.

An edequate heav-ainorul fraction cuild be obtaina fron theso conposito sampleb. The fracts on was quarteron and nounted in a suitiolo al1de with Canada baloain $(k=1.54)$ or arocitor $(1=2.66)$. 


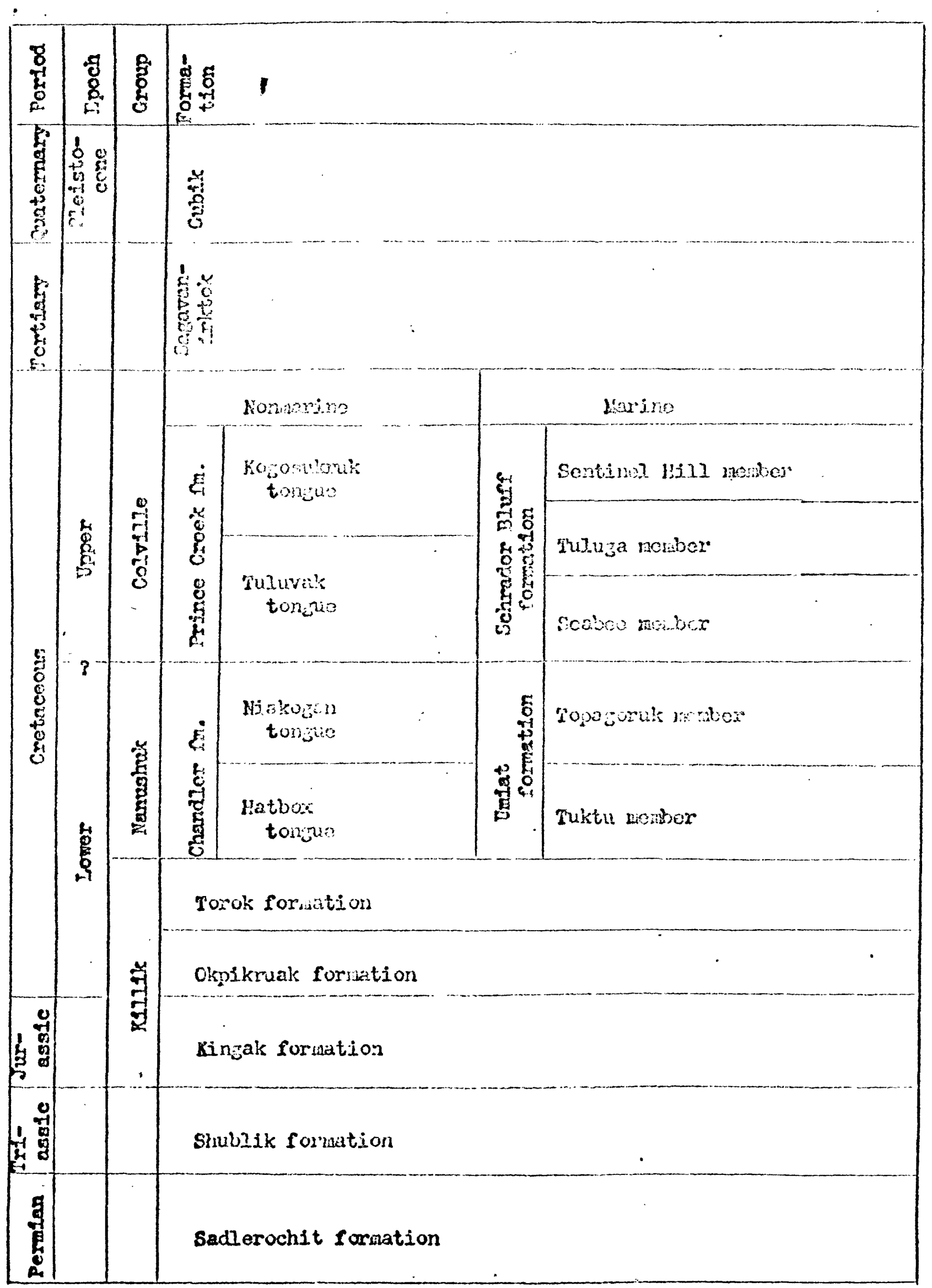

Table 1. 8tratigraphlc names of post-Carbonifercus rocks 


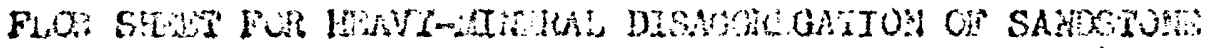

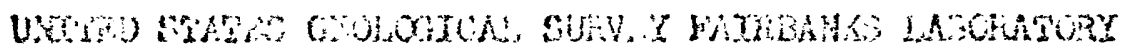

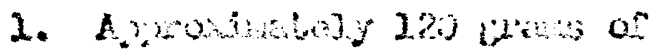

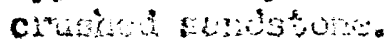

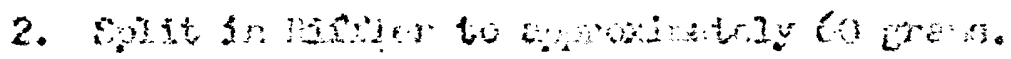

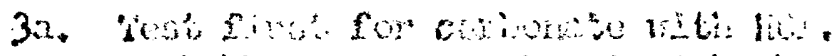

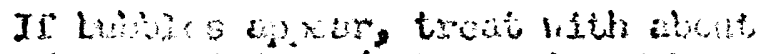

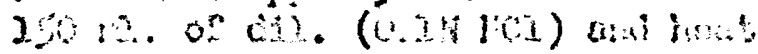

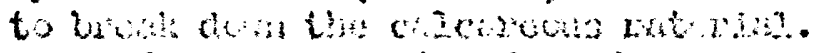

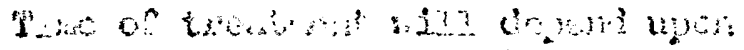

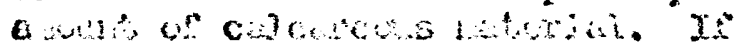

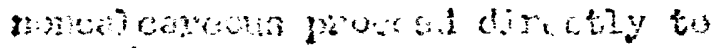
sits 5 .

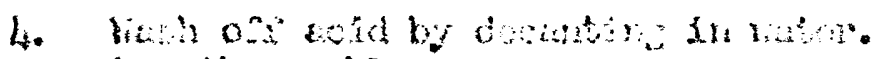

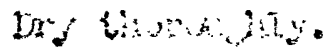

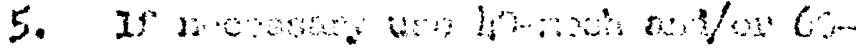

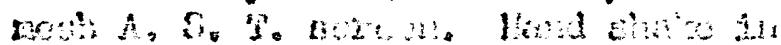

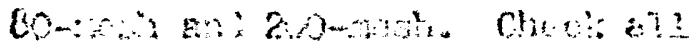

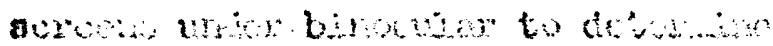

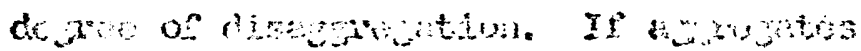

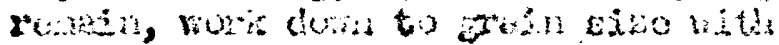

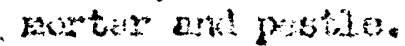

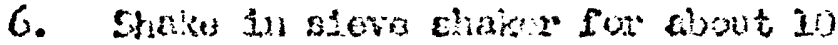
alratos.

7a. Combsn the ; 80 - wowh ain $+200-50$ sh fraction in a laboi ad bealox.

70. Dascard fraction in tho pesl.

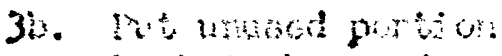
bost in bag and 2 2id.

8. Test for calcarous content. It any, recat treatint whit Hil acid as in olep 3ix.

9. Royeat thorudith washing and ery 1:? beuker or filter piepel.

lleady for bromofura sojalyition. 


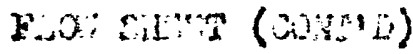

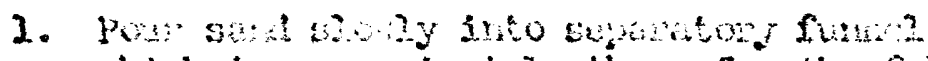

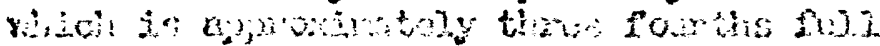

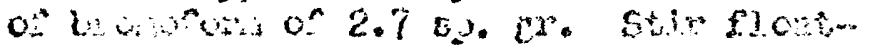

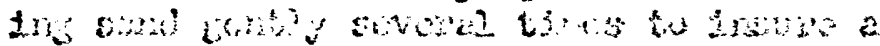

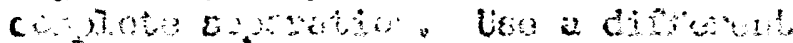
5. cotention

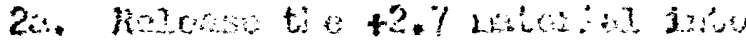

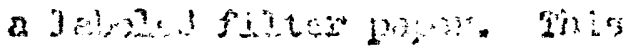

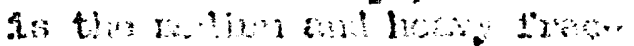

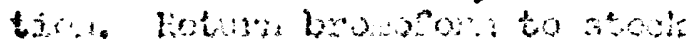
botita.

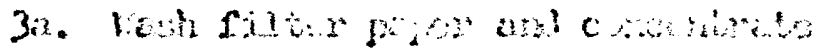

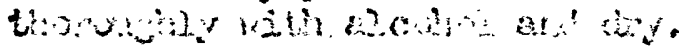

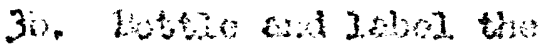
21046 frotion.

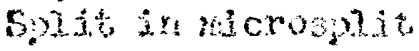
if $r$ ander.

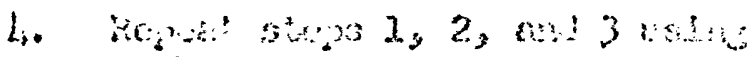

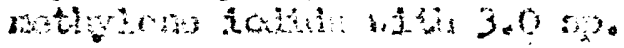
6.

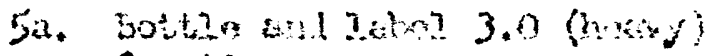
frection.

55. Dotrin at $12401+2.7$ -3.0 (nobus) iractio.s. 


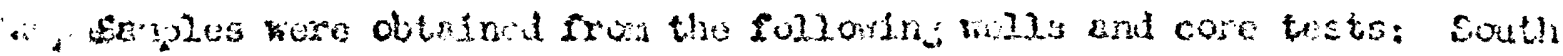

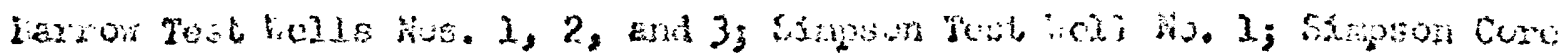

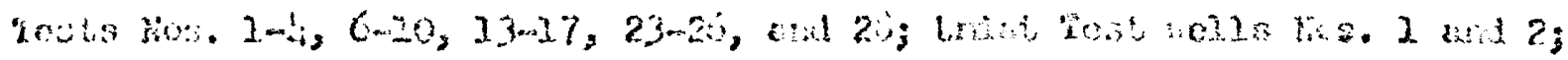

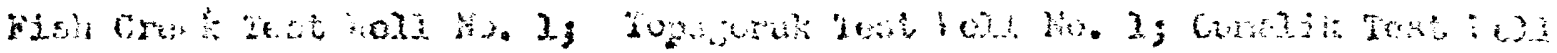

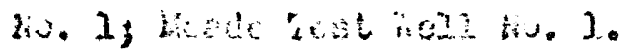

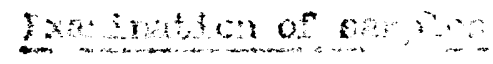

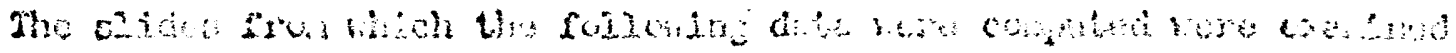

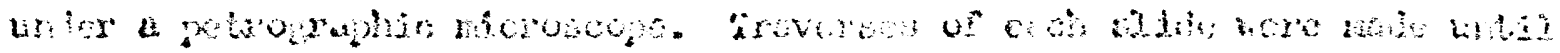

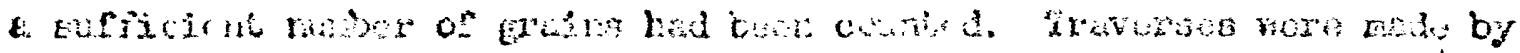

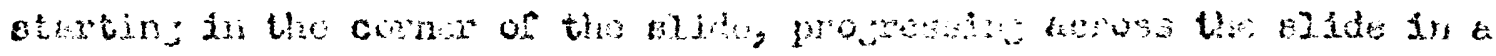

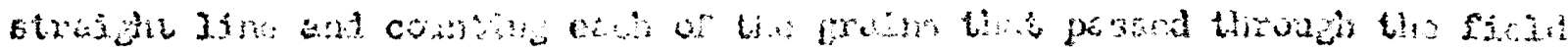

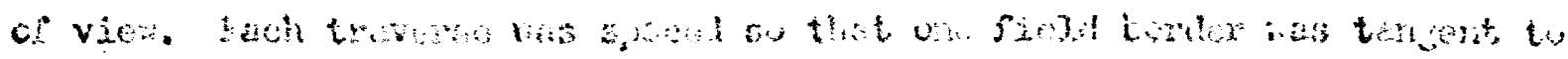

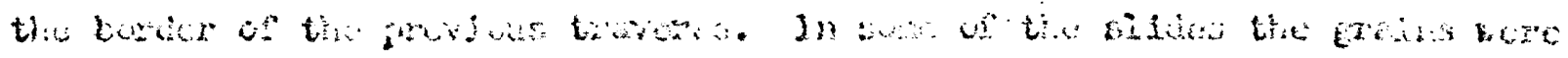

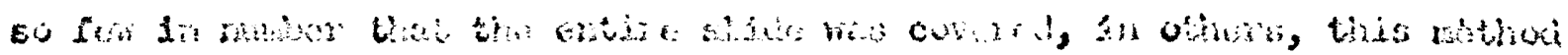

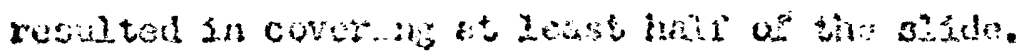

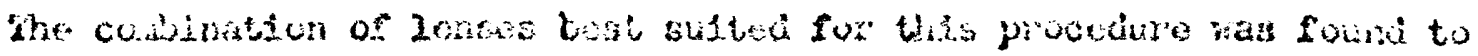

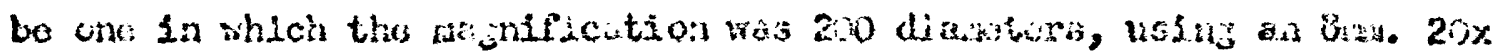

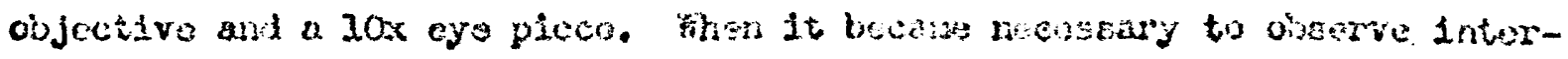

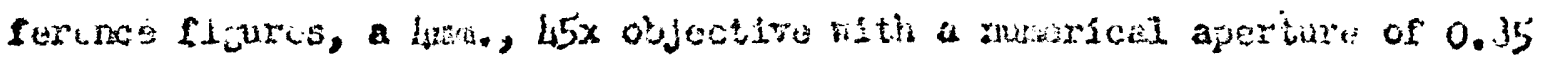
was uect.

\section{Mris $\operatorname{cosin} \theta$}

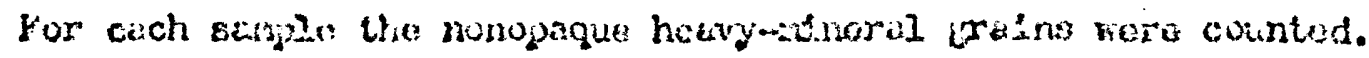

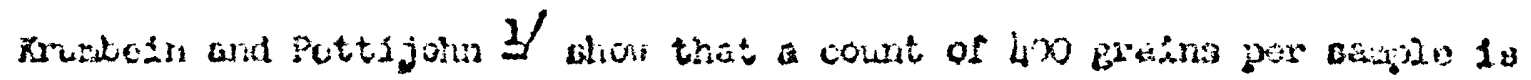

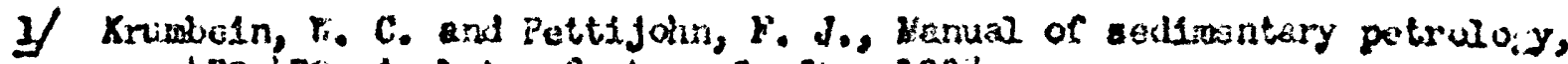
Pp. 470-L72, Apjetor-Centurj-Crurts, 2936. 


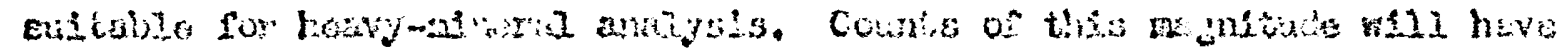

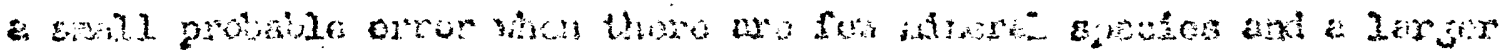

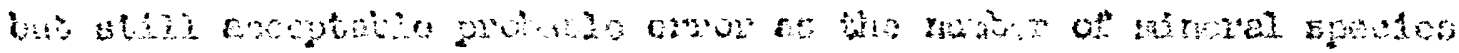

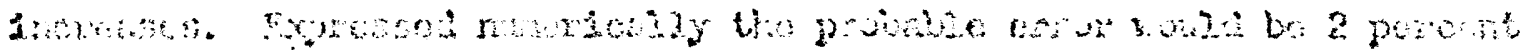

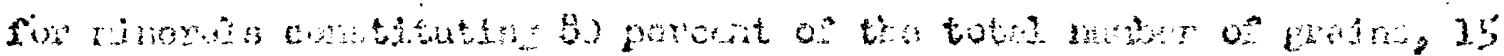

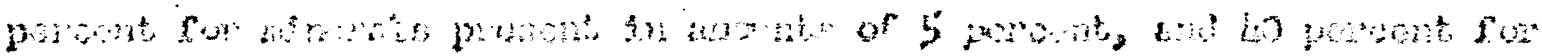

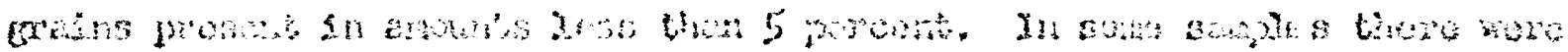

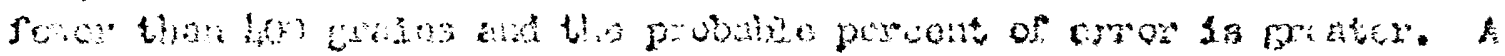

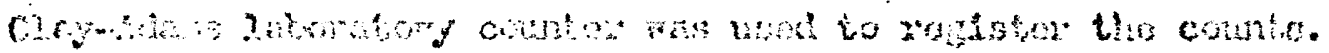
tenters

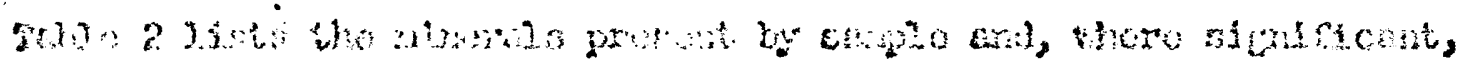

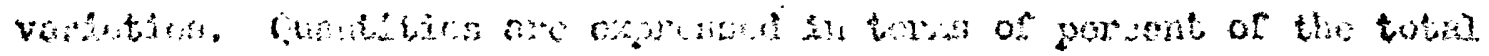

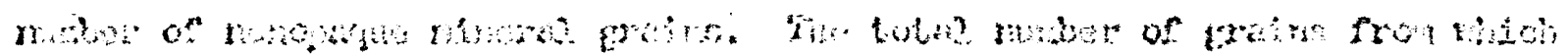

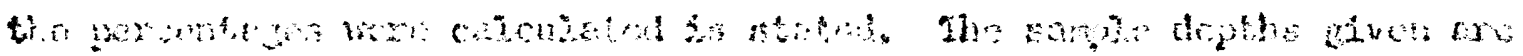

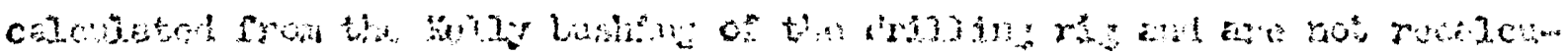

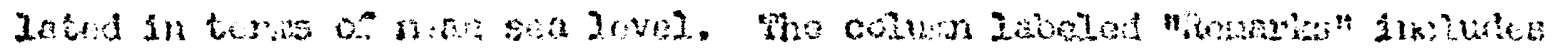

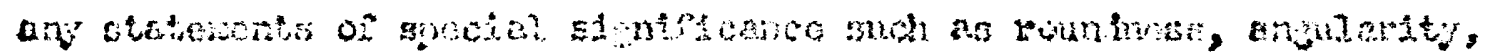

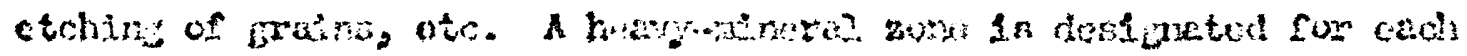

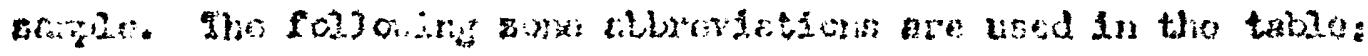

\section{0}

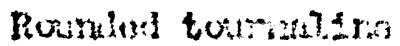

Fris atsc tourajing

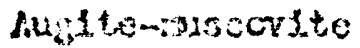

zored aireor.

Euhrolit asacon

Ciaucu, hano-idornhlemb

BLutits

liourled grasn
Abingsiation

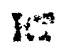

25

$A \rightarrow-\infty$

$2 \%$

12

Gi-it

B

III 
Table 2. Nicavy mincrus conosition of wols saglos by pareents

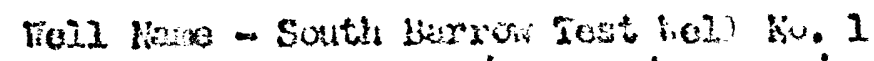

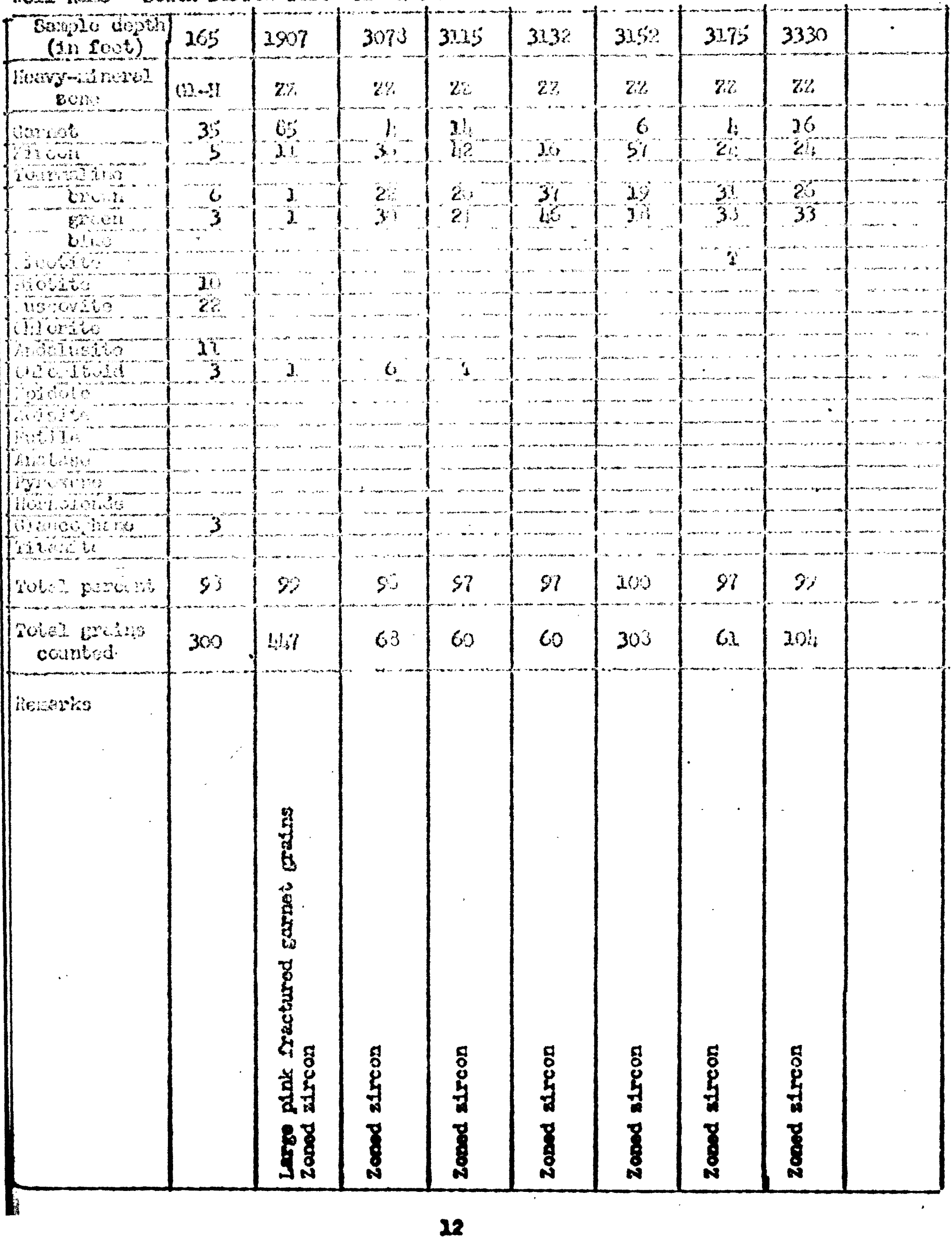




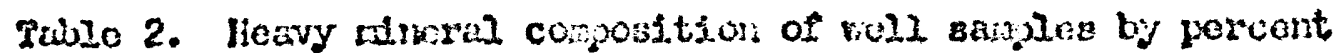

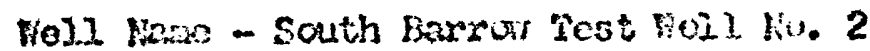

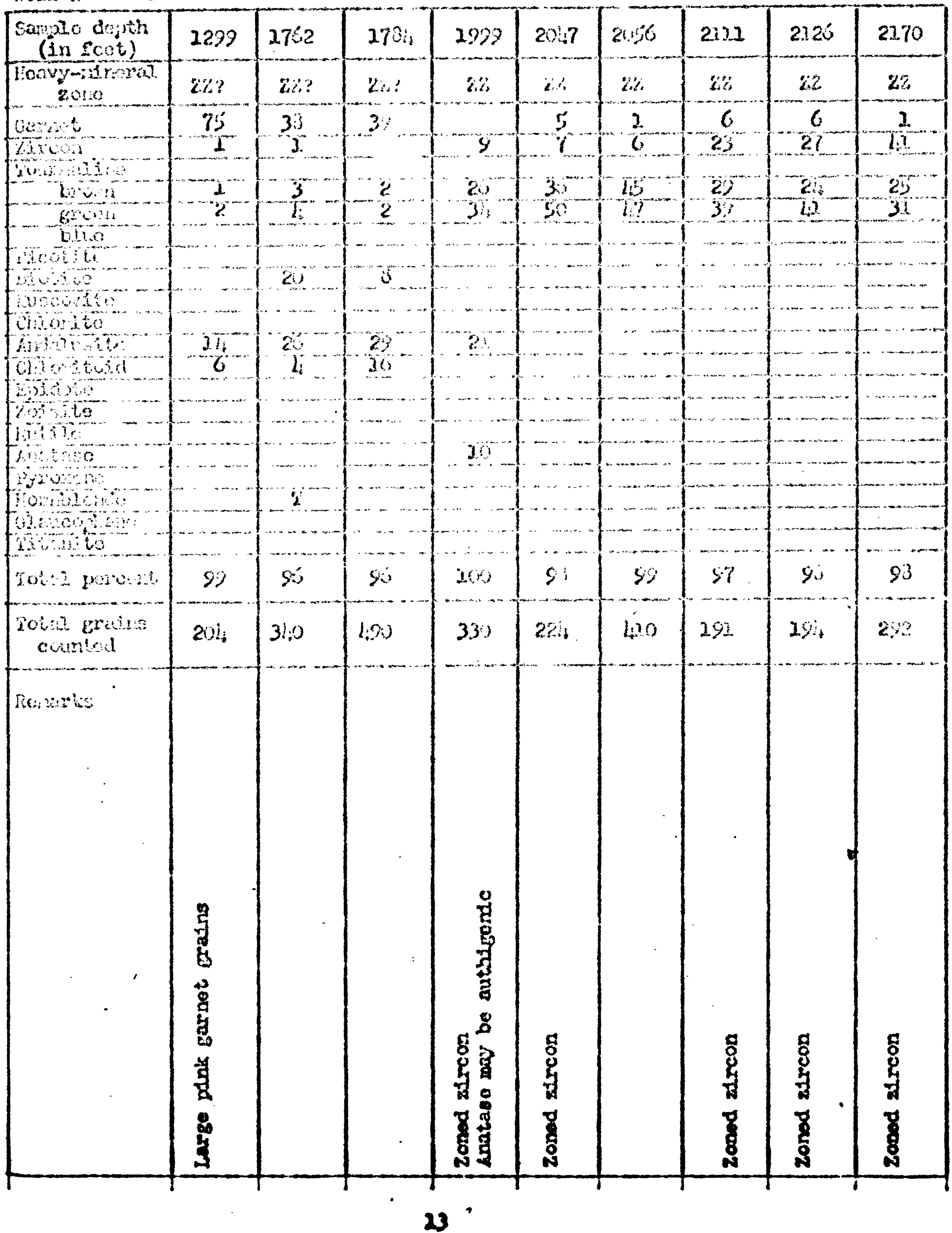




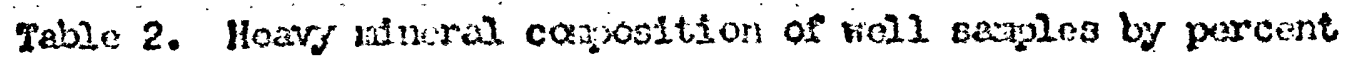

Fell keno - South Rarrot Test ind ko. 2 (conth)

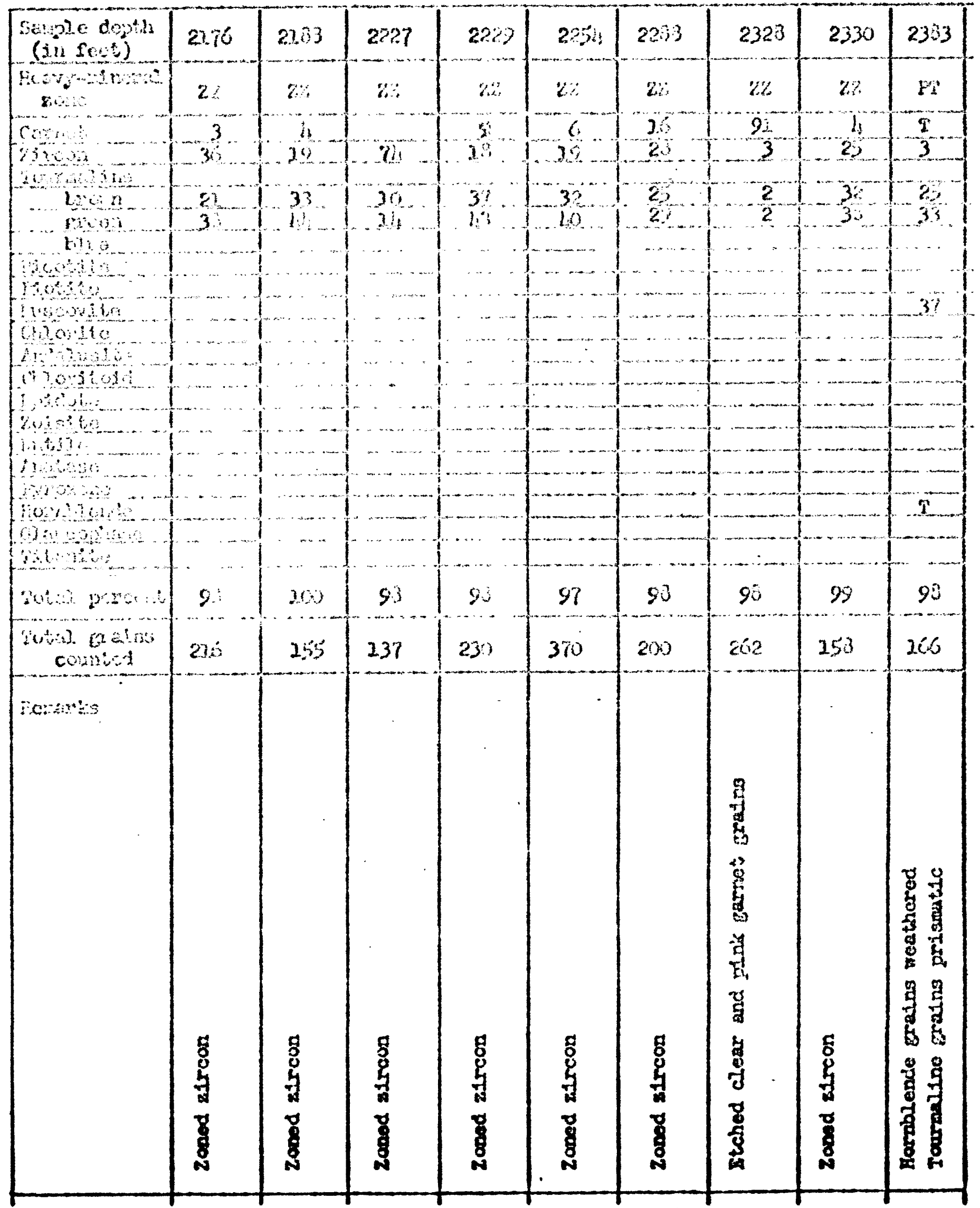




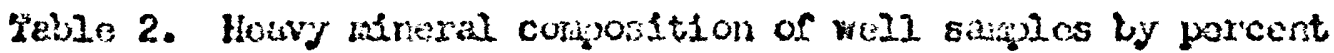

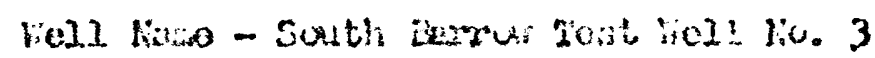

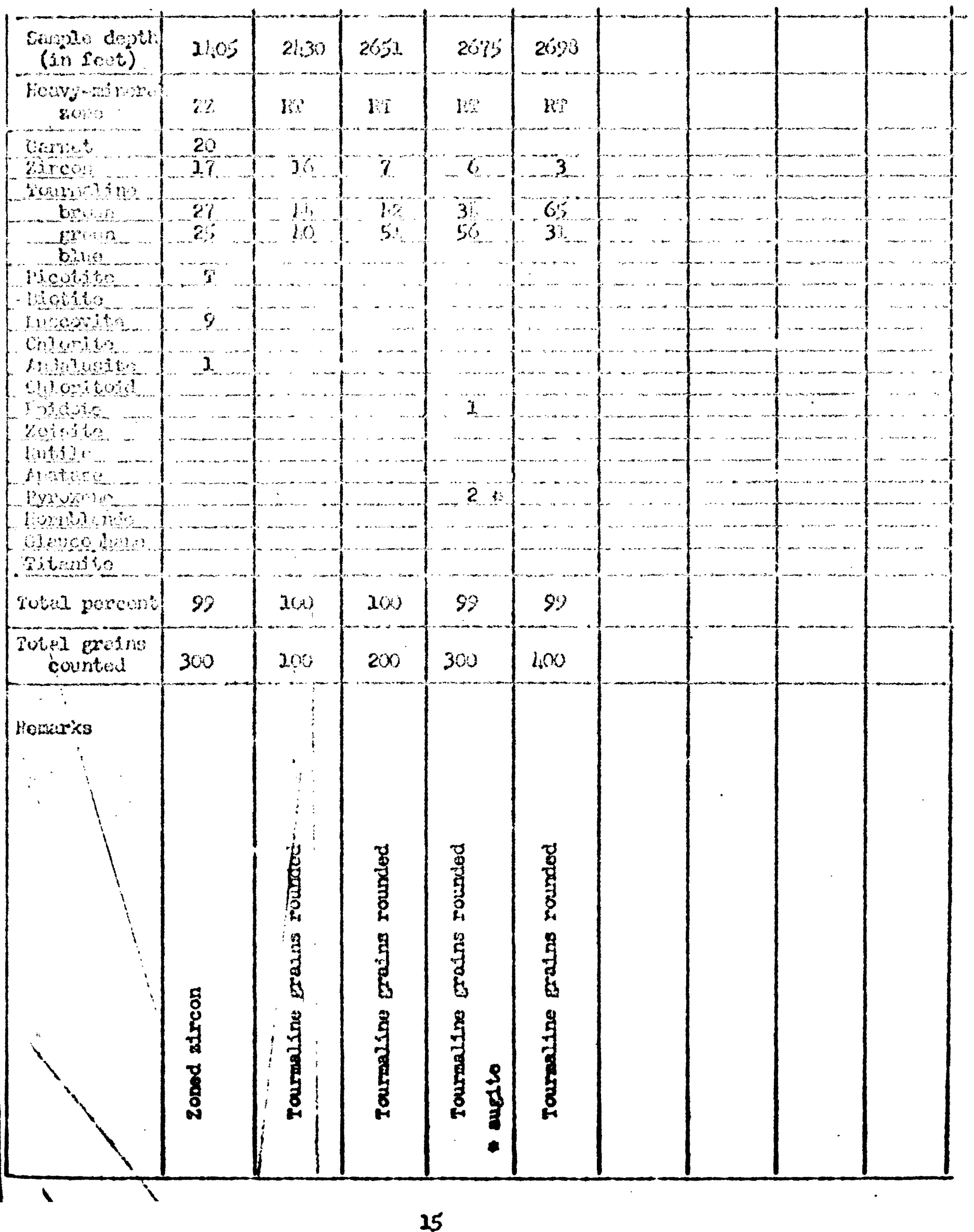


Tablo 2. Hoavy ninoral couplosition of well eanlles by percert

Voll Kax - Simpson Tost roul Ho. 1

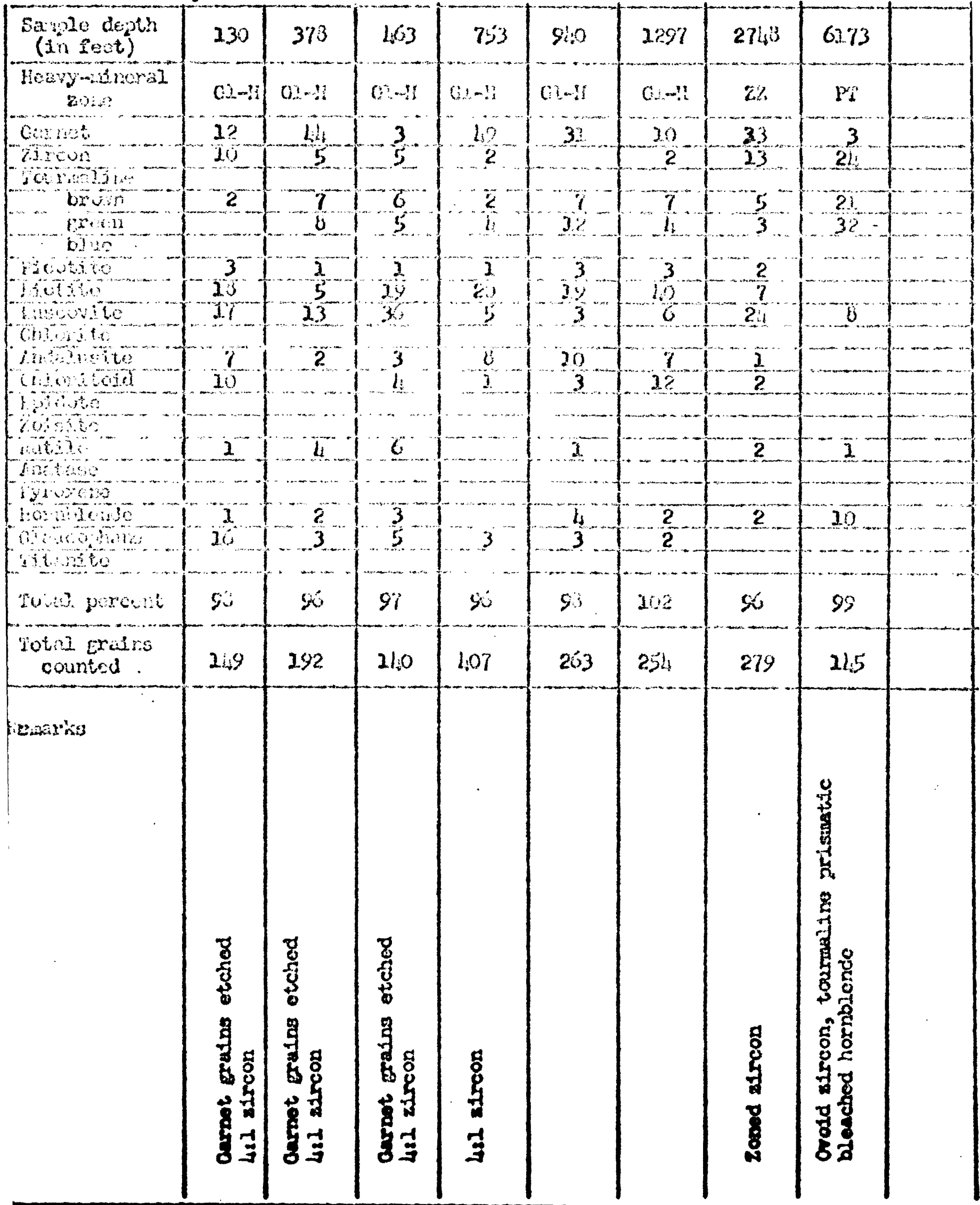


Table 2. Heavy noworal cospbition of nell saiglos by percent

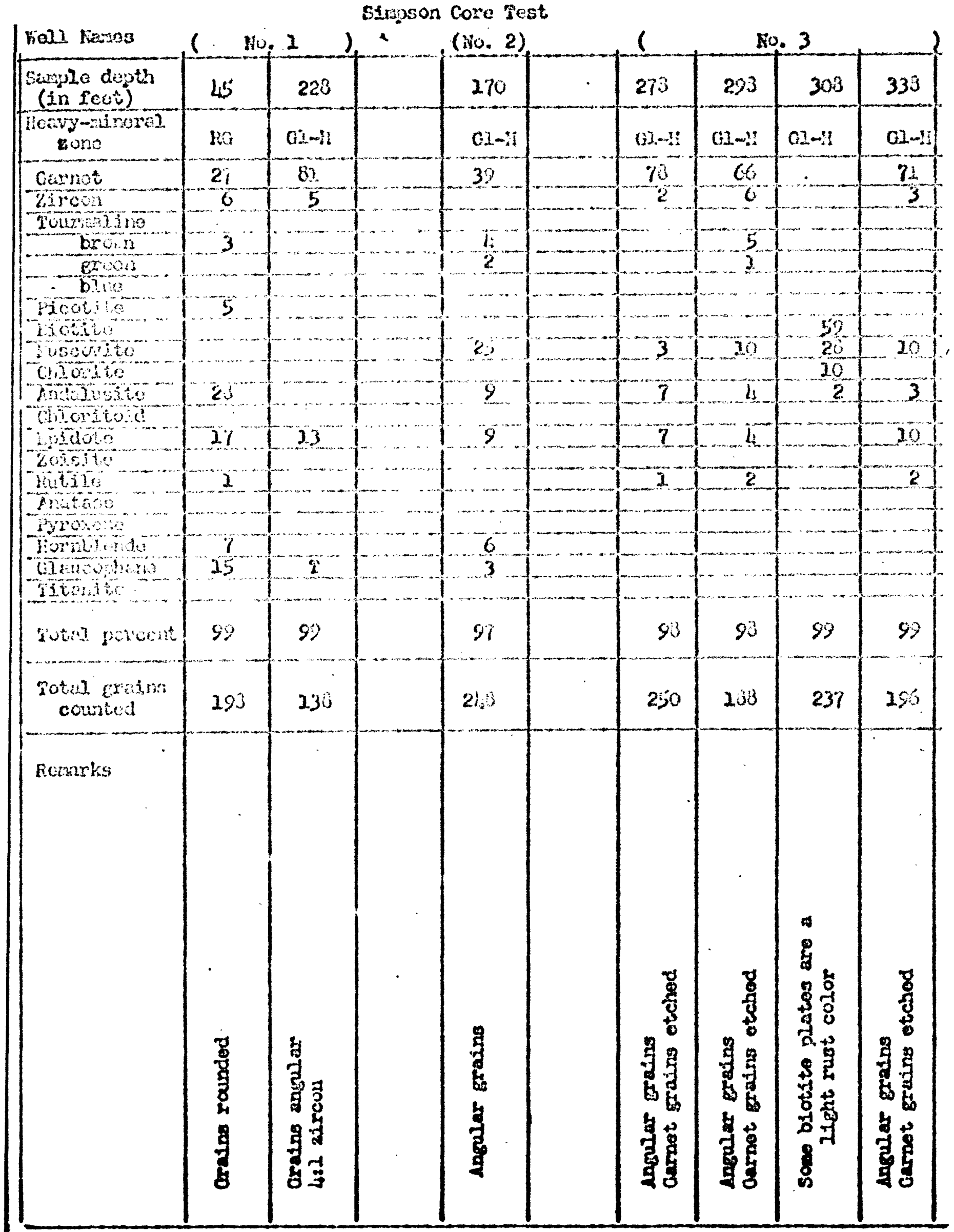


Tablo 2. llecks afroral composition of vall sagles by percent

\begin{tabular}{|c|c|c|c|c|c|c|c|c|c|}
\hline $\begin{array}{c}\text { Samole dojt } \\
\text { (in } f(u t)\end{array}$ & 01 & 103 & 121 & $i$ & 29 & $\cdot 45$ & 229 & & 372 \\
\hline 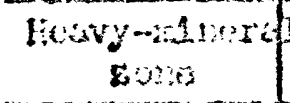 & $(2-2 !$ & $\mathrm{G}=1$ & $02-3$ & & $\mathrm{kic}$ & 10 & $01 . \cdots 1$ & & Q.2.:-: \\
\hline Gerget & $\omega$ & & 11 & & 22 & II & 35 & & 16 \\
\hline $2 \operatorname{sen}$ & 2 & $\ldots .$. & & & $I$ & & 2 & & 3 \\
\hline Tuinging & $\cdots$ & & & & & & & & \\
\hline bon & $\ldots$ & ………... & $-\ldots . . . . . .$. & & .... & 2 & 1 & & $l_{i}$ \\
\hline Crus & ...... & ......... & ......... & & 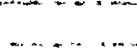 & ..... & . & $\ldots$ & $\ldots$ \\
\hline 810 & -... & .... & ……n. & . & $\cdots$ & ......... & & & \\
\hline Povobed & & & & 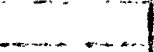 & $\cdots$ & & & & \\
\hline Distig & 2 & $2 !$ & 2 & & 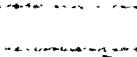 & & $E$ & & $3 !$ \\
\hline Wuosote & 2 & 27 & 2 & & 1 & ...... & 20 & & $3 y$ \\
\hline onjosite & & & & & 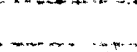 & $\ldots-\infty$ & & $\ldots$ & $\ldots \ldots$ \\
\hline Andustie & 5 & 13 & 20 & & $\ldots \ldots$ & $2 i$ & 32 & & 21 \\
\hline Chioritus & 1 & 7 & 2 & & & & 2 & 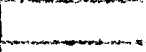 & \\
\hline 60060 & 15 & 30 & 41 & $\ldots$ & $0 \%$ & $2 y$ & 3 & & \\
\hline 201510 & $\infty$ & & & & ……..... & & & & \\
\hline llatio & 2 & $\ldots \ldots$ & 20 & & ............... & 2 & 2 & & 2 \\
\hline MnElese & $\ldots$ & …...... & ..... & & 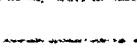 & & & & \\
\hline Dyruxone & $1 *$ & thes & 2 & & 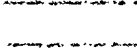 & & & & \\
\hline Hordulends & 1 & 3 & 29 & & 2 & 22 & 2 & & \\
\hline G] tancondes & 2 & 13 & 2 & & $\ldots$ & 13 & 14 & & 2 \\
\hline Tatests & 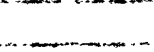 & & $\ldots \ldots$ & & - …- & & $\ldots$ & & \\
\hline potal $p+3+4$ & 893 & 97 & 202 & & só & 93 & 95 & & 97 \\
\hline $\begin{array}{l}\text { rotel eraing } \\
\text { combited }\end{array}$ & 300 & 21,0 & 200 & & 35 & 300 & 333 & & 21,7 \\
\hline Re:zarks & & & & & & & & & \\
\hline & 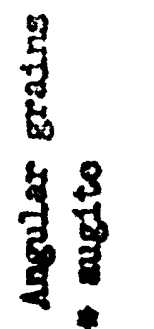 & 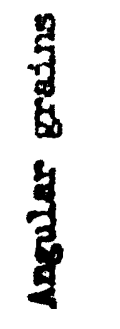 & 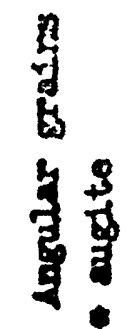 & & 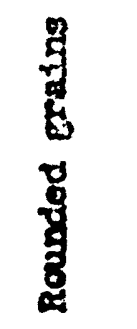 & $\begin{array}{l}\frac{5}{8} \\
8 \\
8 \\
8 \\
8 \\
8\end{array}$ & 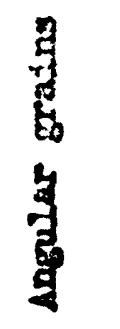 & & 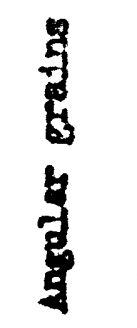 \\
\hline
\end{tabular}


gablo 2. lleavy bineral compostion of rell samplos by percent

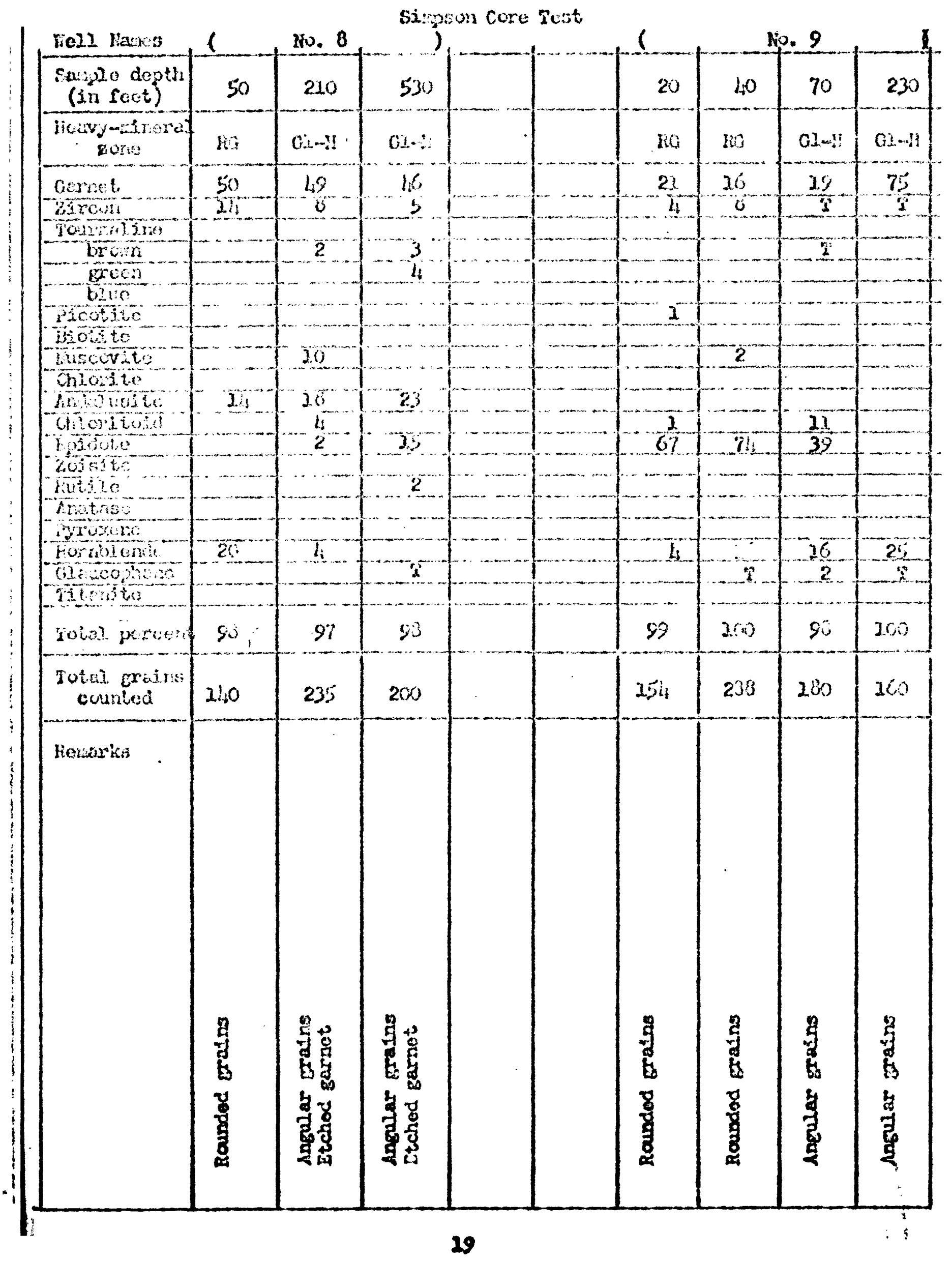


Tabie 2. Heavy ninerai conposition of woli bapslins by percent

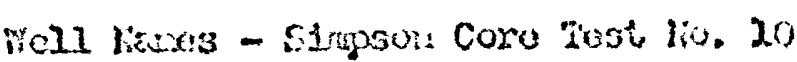

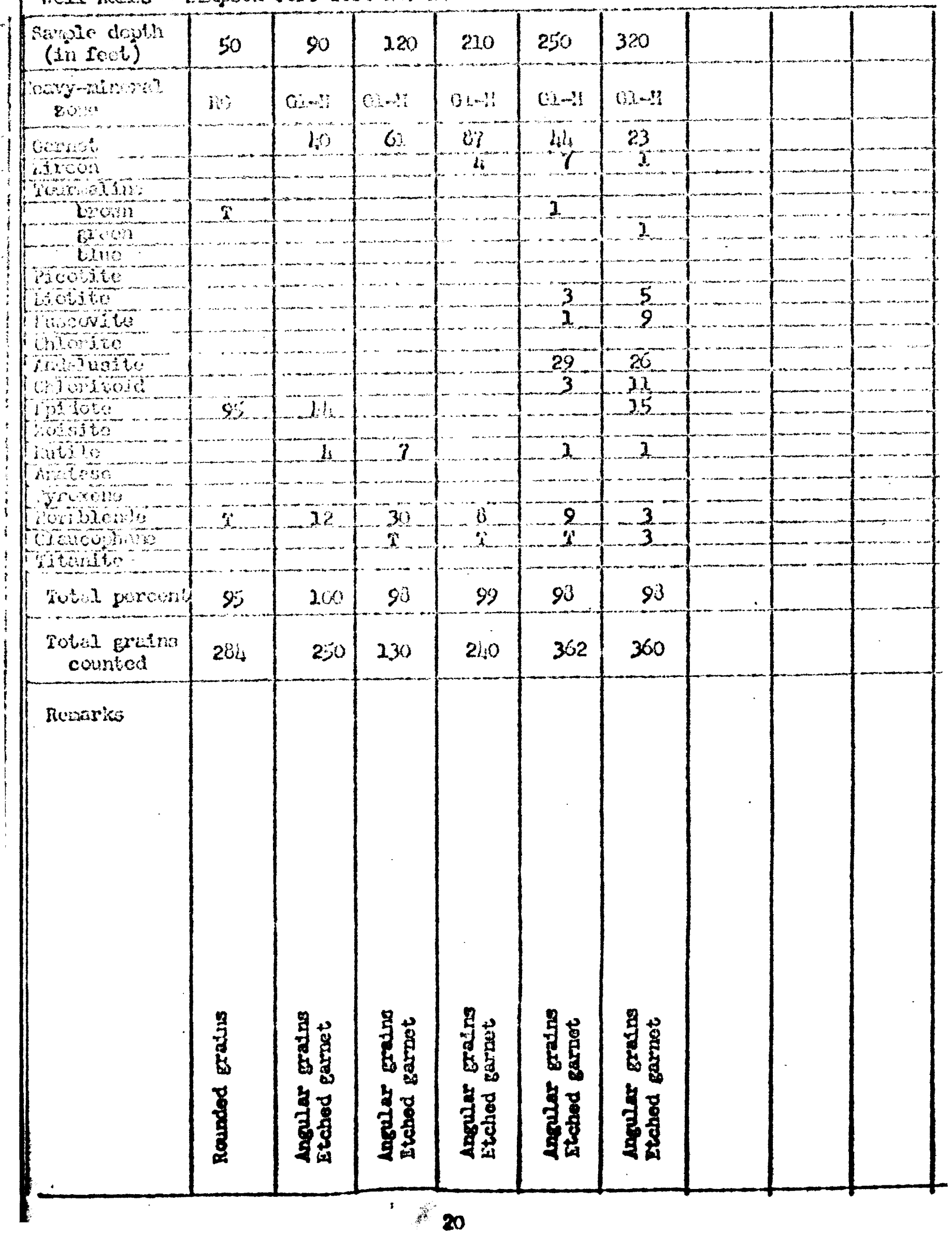


Tablo 2. Hecvy miroral copposition of roll sajiles by percent i

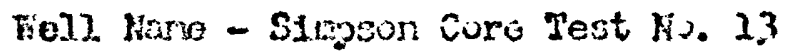

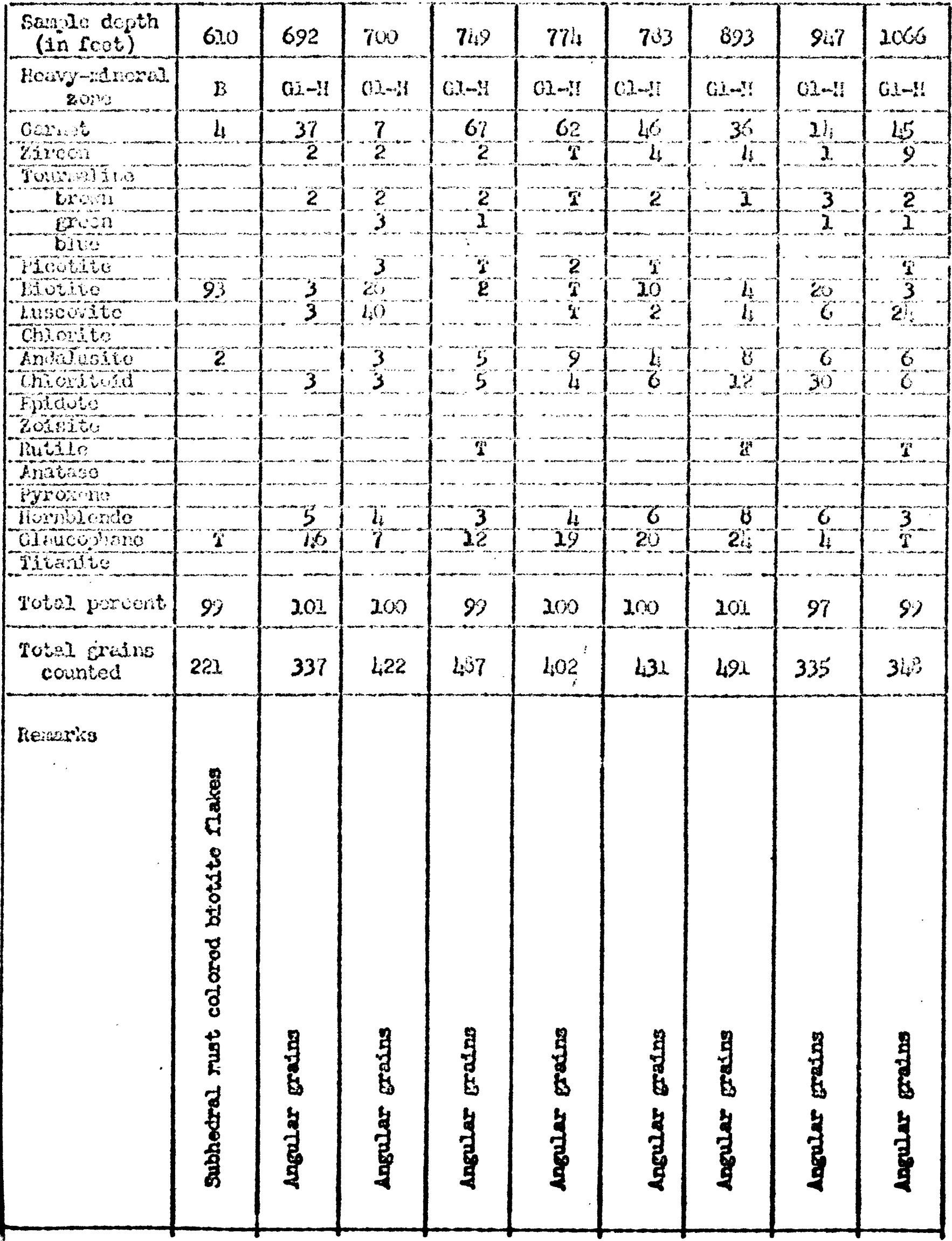


Table 2. Hexvy minoral composition of roll gajles by pareunt

Foil karo - Simpson Curo Test Hu. 23 (cont'd)

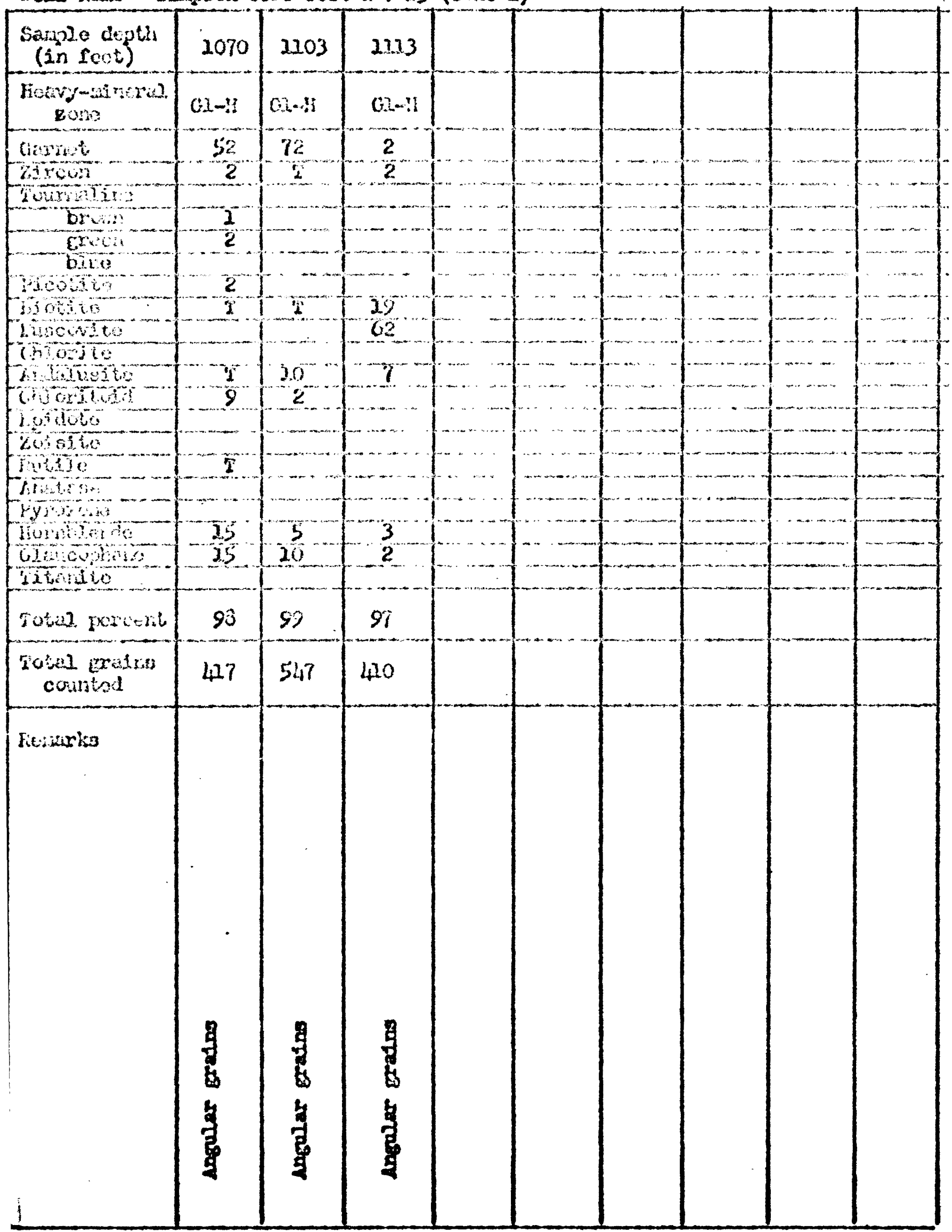


Table 2. Foavy minerial conjosition of weIl earjles by percont

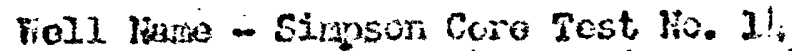

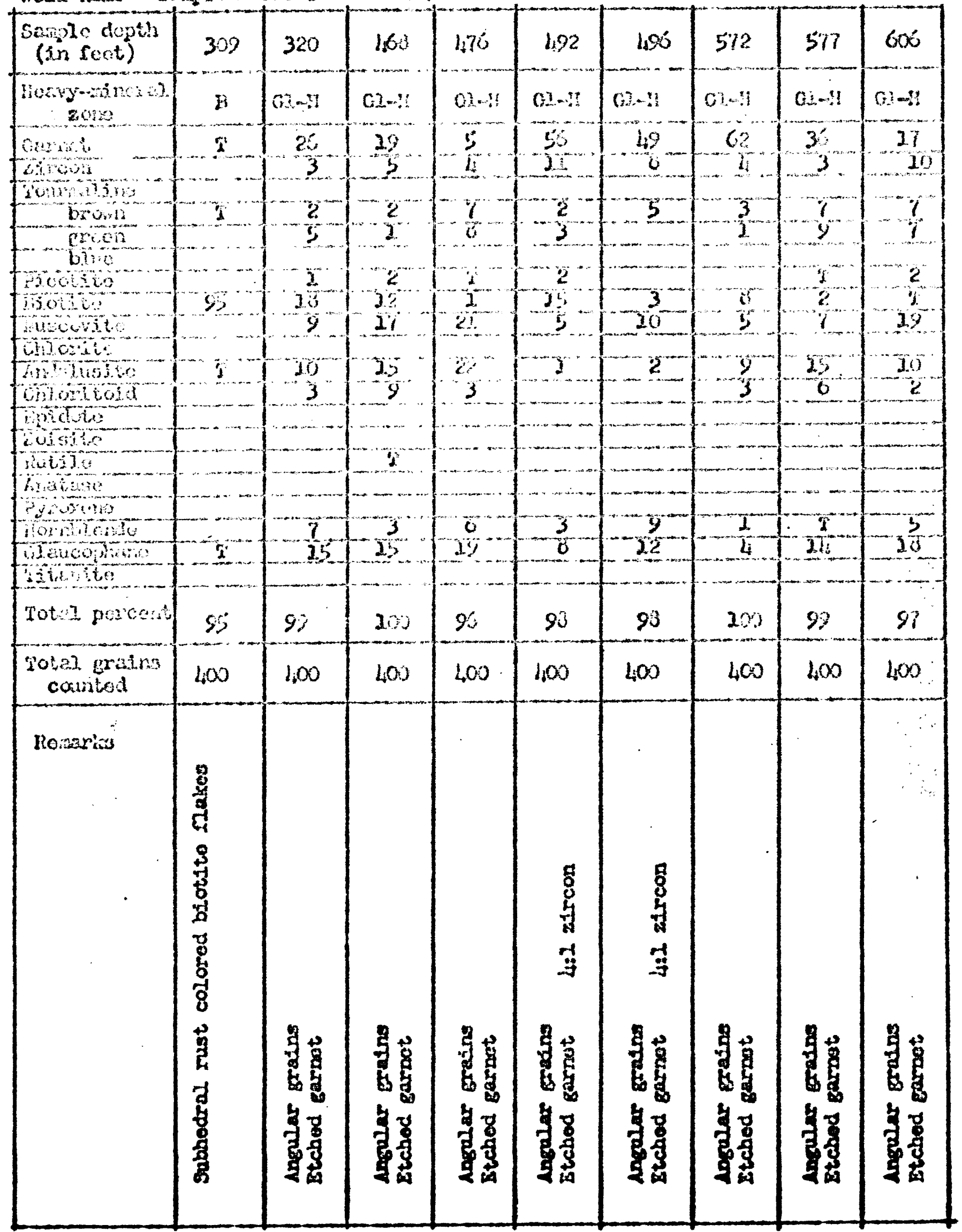




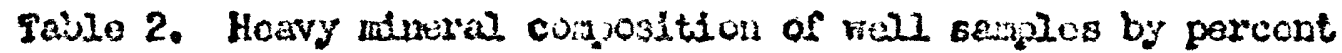

Foll Namo - Simgan Cure Tost lio. lis (cont'd)

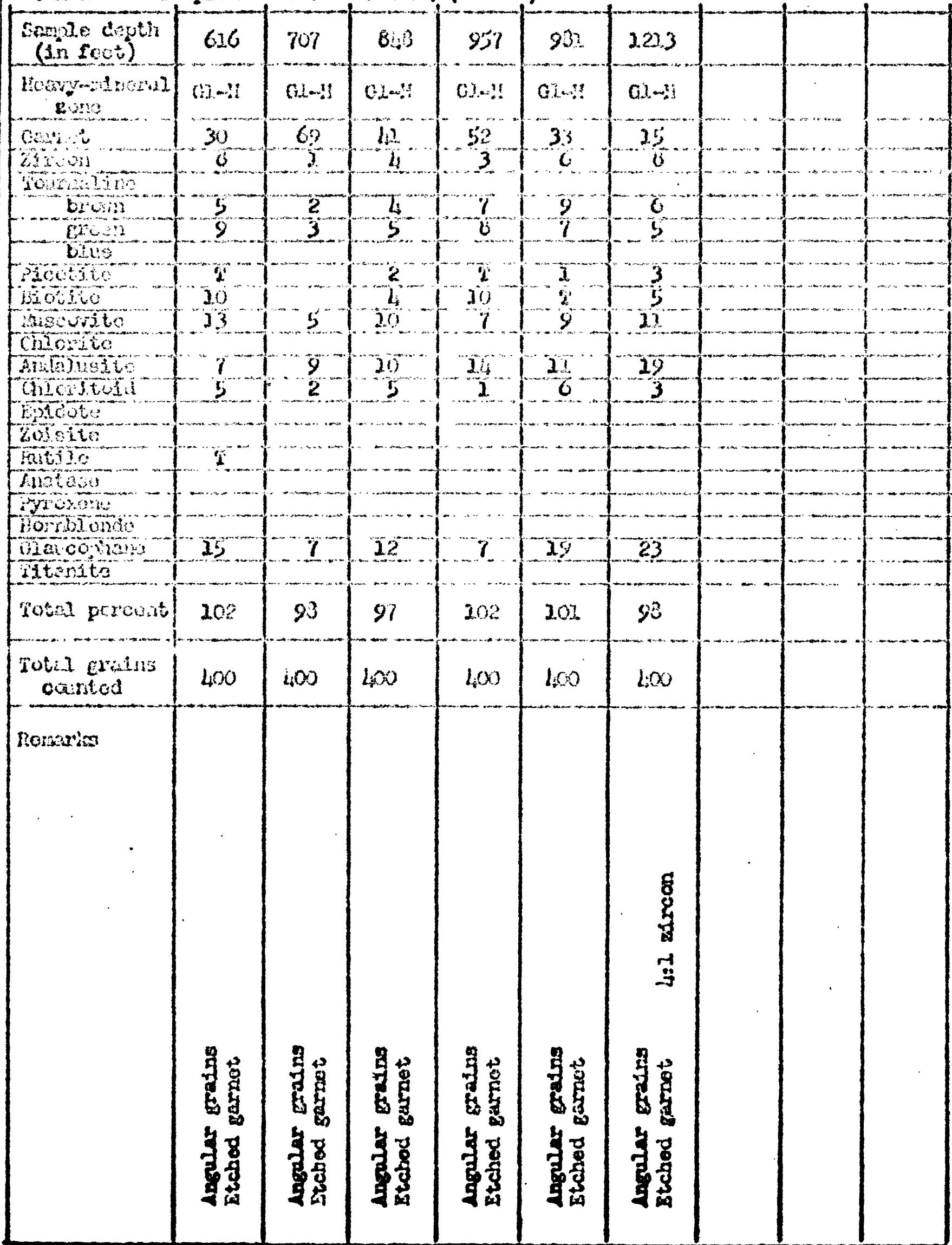


Table 2. Heavy winexal congegltion of well aajules by porcent

Sinjosin Core Test

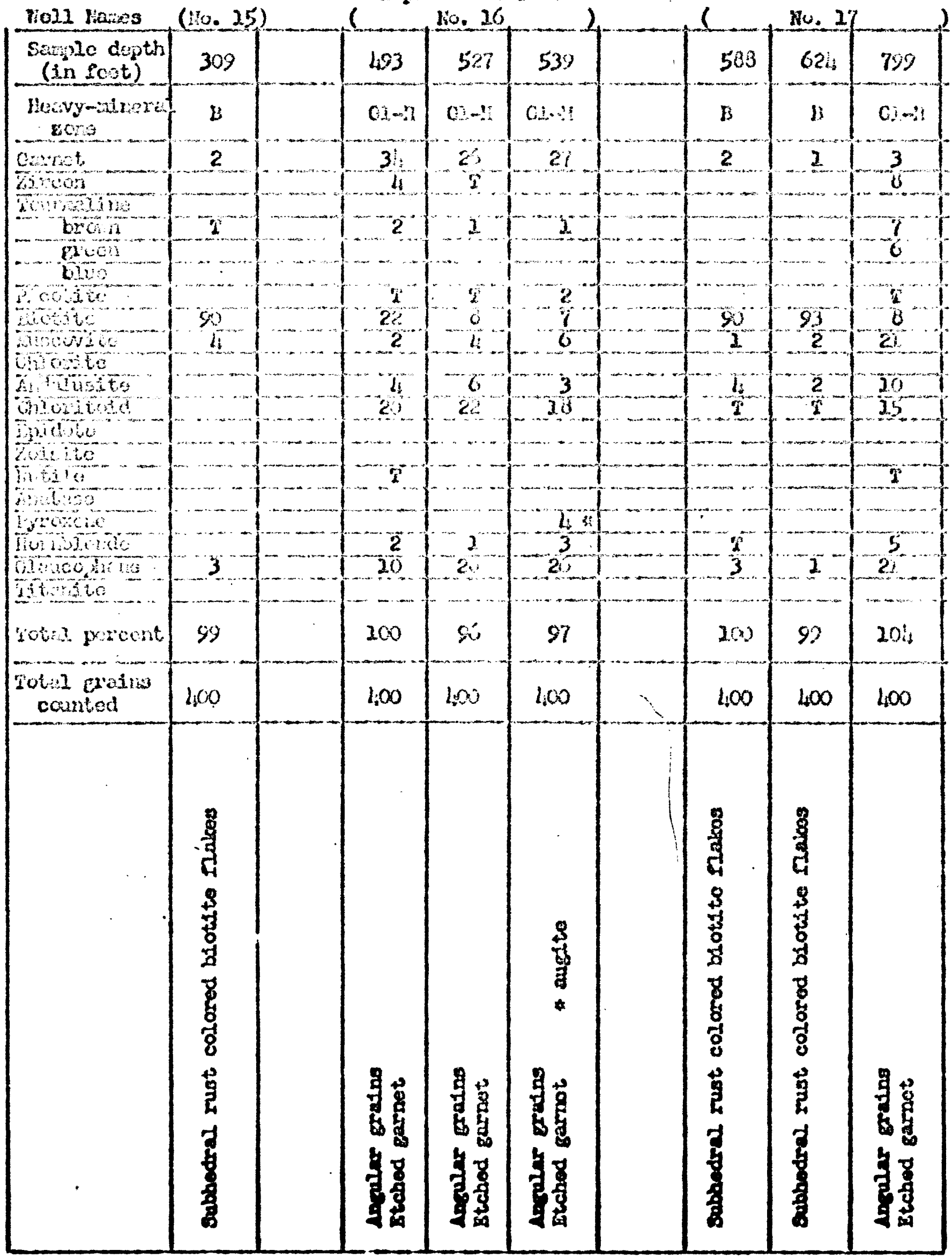


Table 2. Heavy nineral cupposition of well sayjles by percent

Fell karas of Siragen Core rest lij. 23

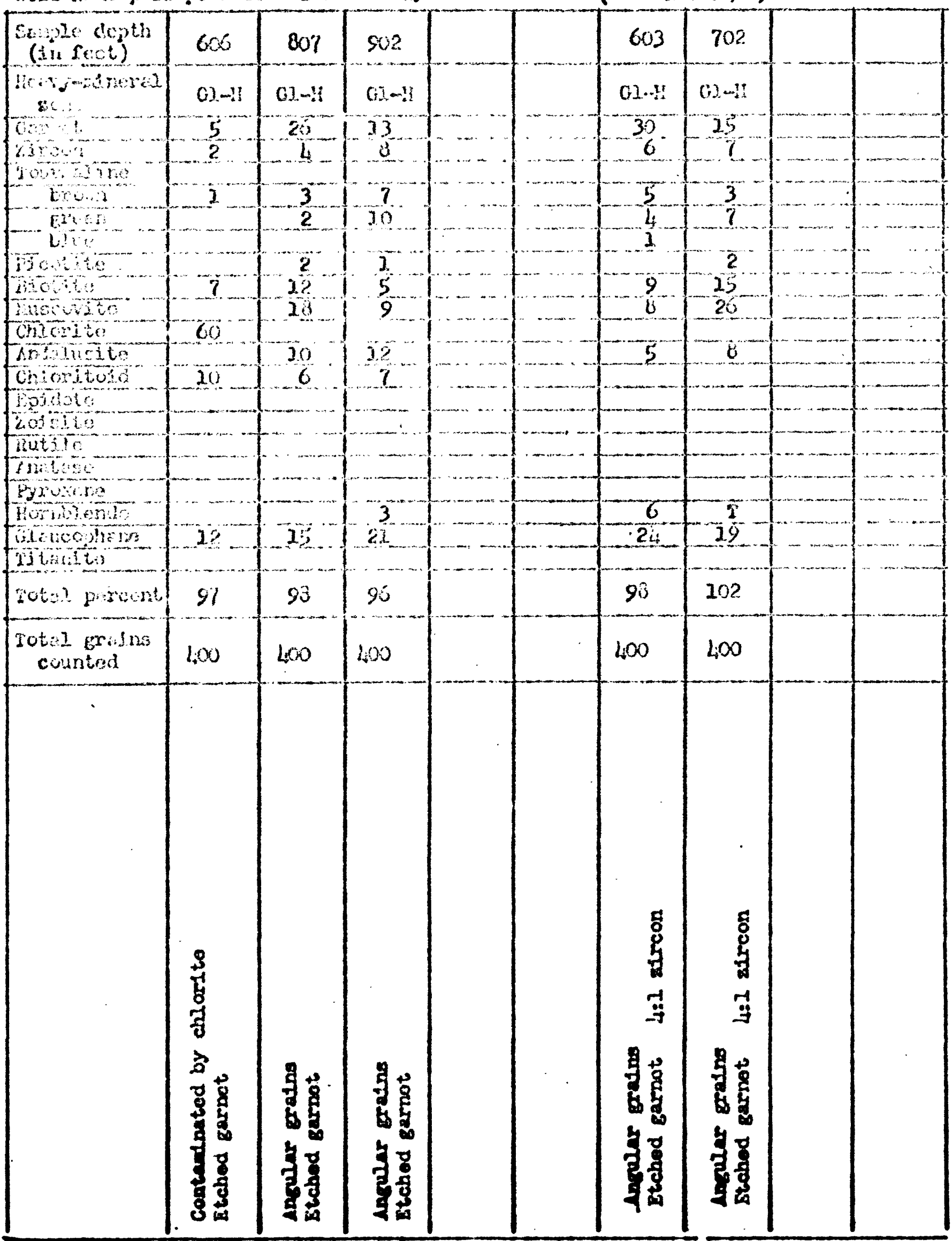


Foble 2. Heavg uneral curyosition of weli Bangles by porcent

Vell Hatra - Sitason core Test H. 25

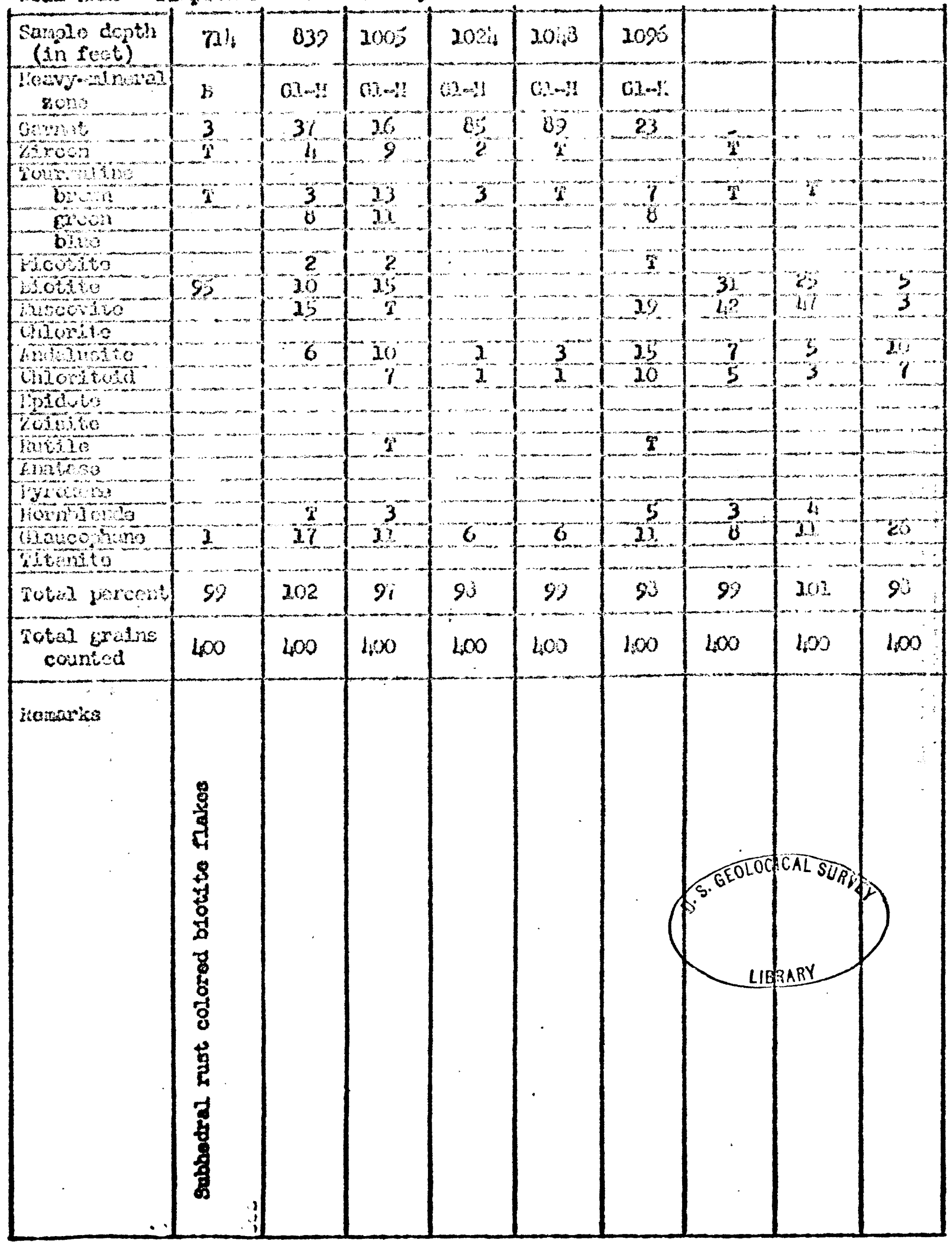


Table 2. Hoavy wineral coarogition of wull eanples by percent

Singown Cores Test

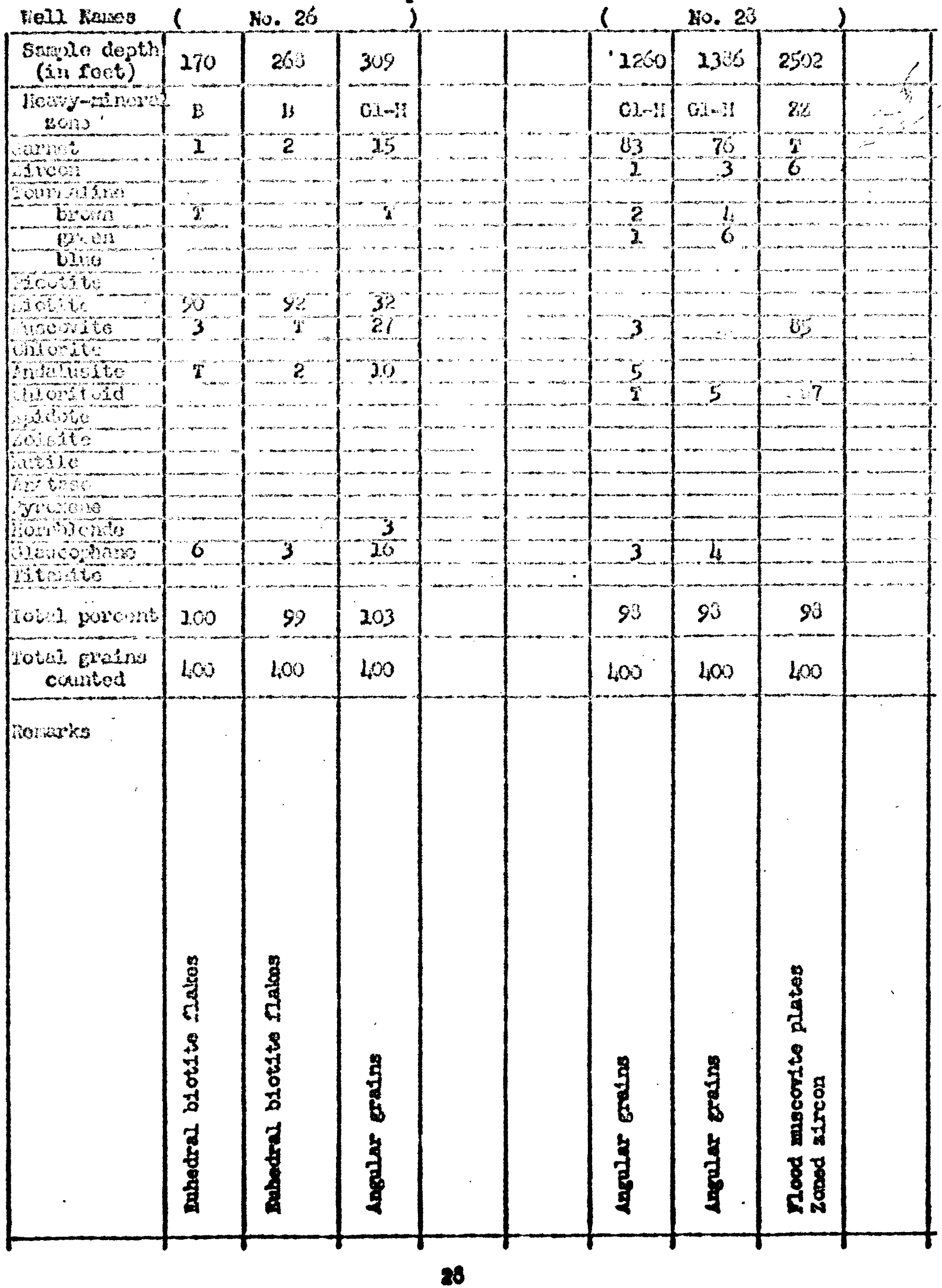


Table 2. Heavy throral conpoustion of woll sasples by percent

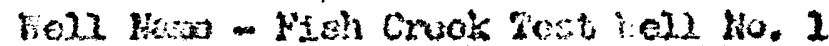

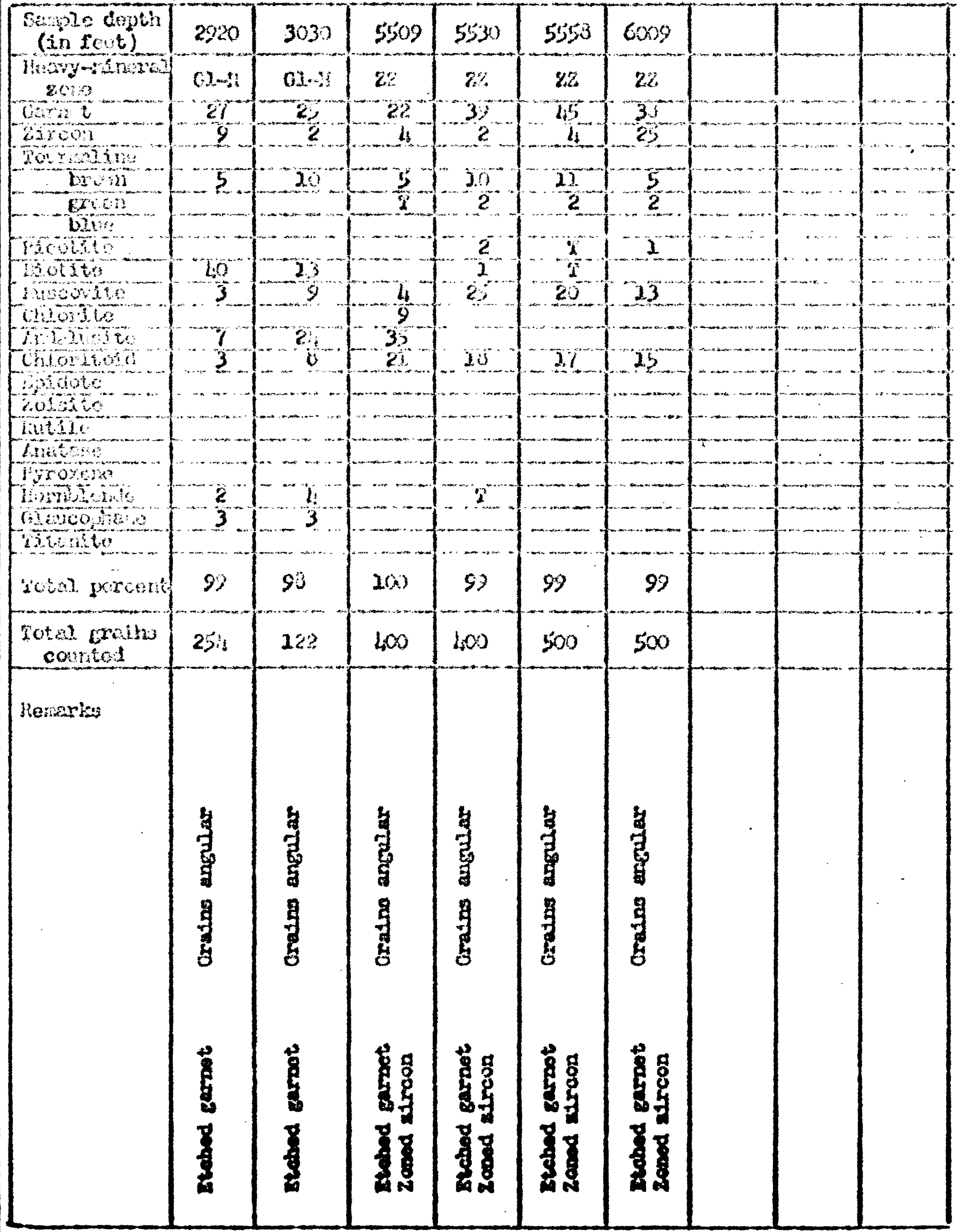


Tablo 2. Heavy minoral conjosition of nell samiles by pereent

Fell Ners - Curalik Test Hedl No. 1

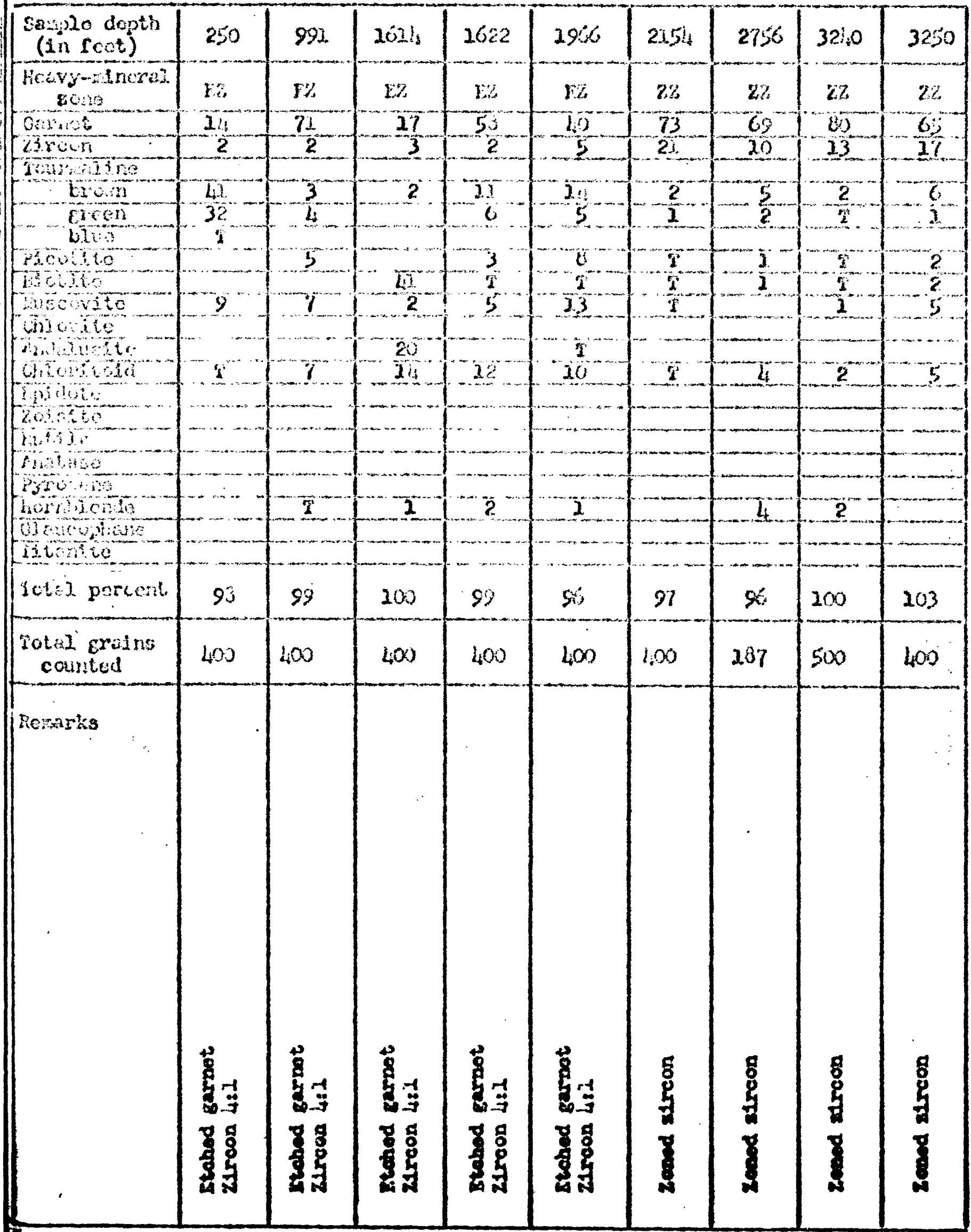


rablo 2. Hoavy zaneral comosition of vell vajples by porcent

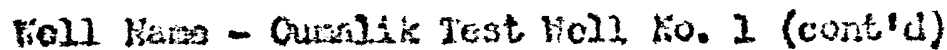

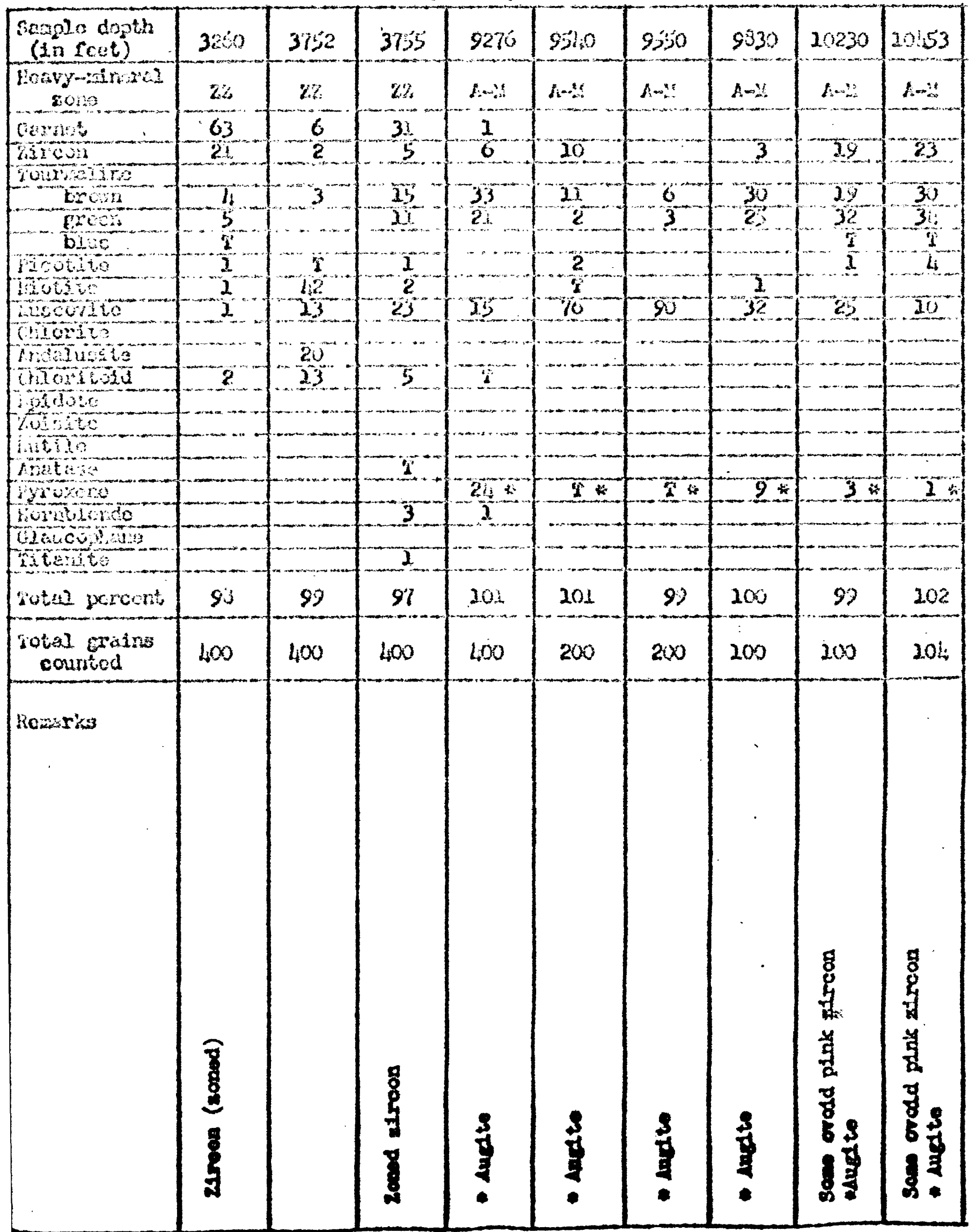


Table 2. Heavy a neral conposition of well aswles in 'percent

Foll la:a - Leade rest Foll No. 1

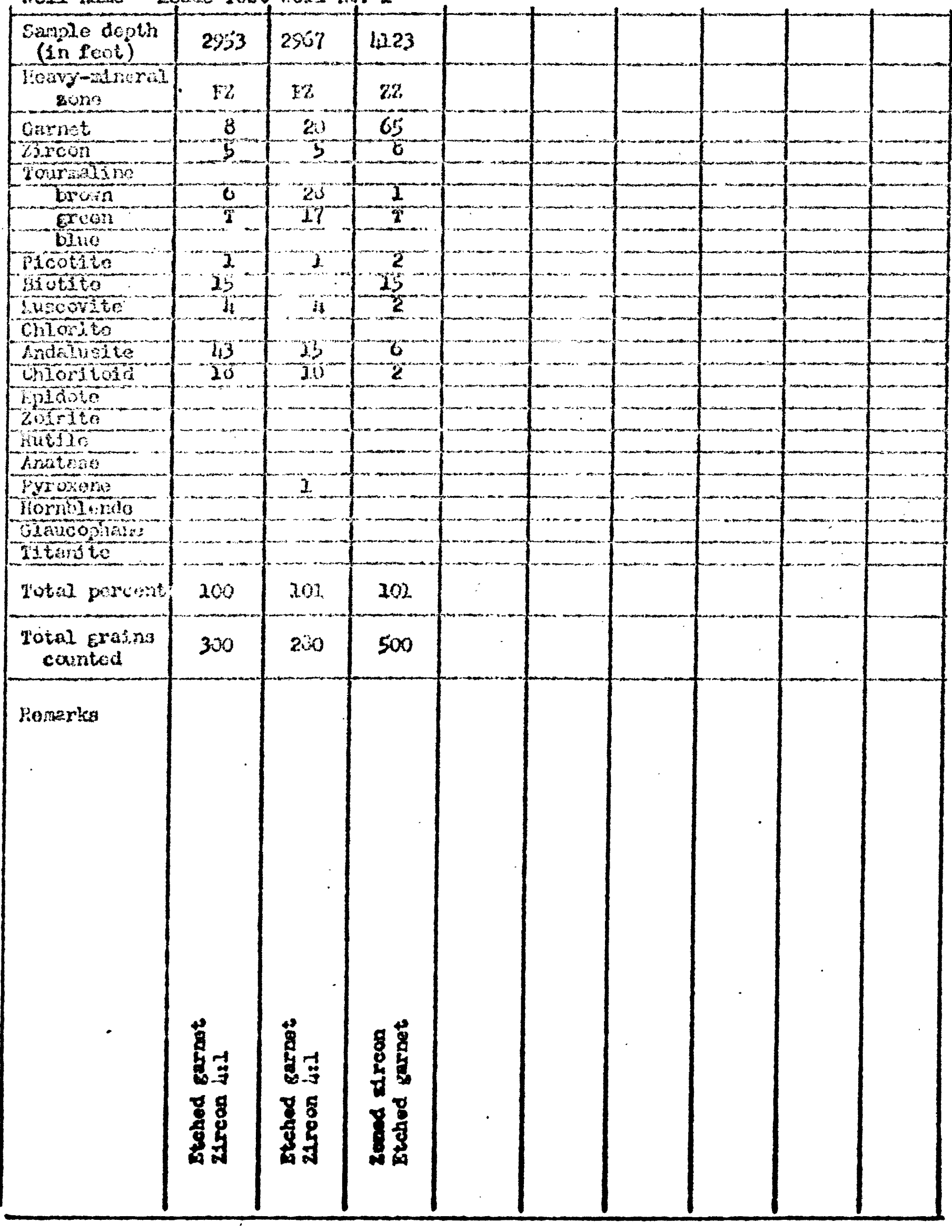


Table 2. Howy minoral corpositios in roll samies by percent

Foll Nars - Topezorut Test relu ko. 2

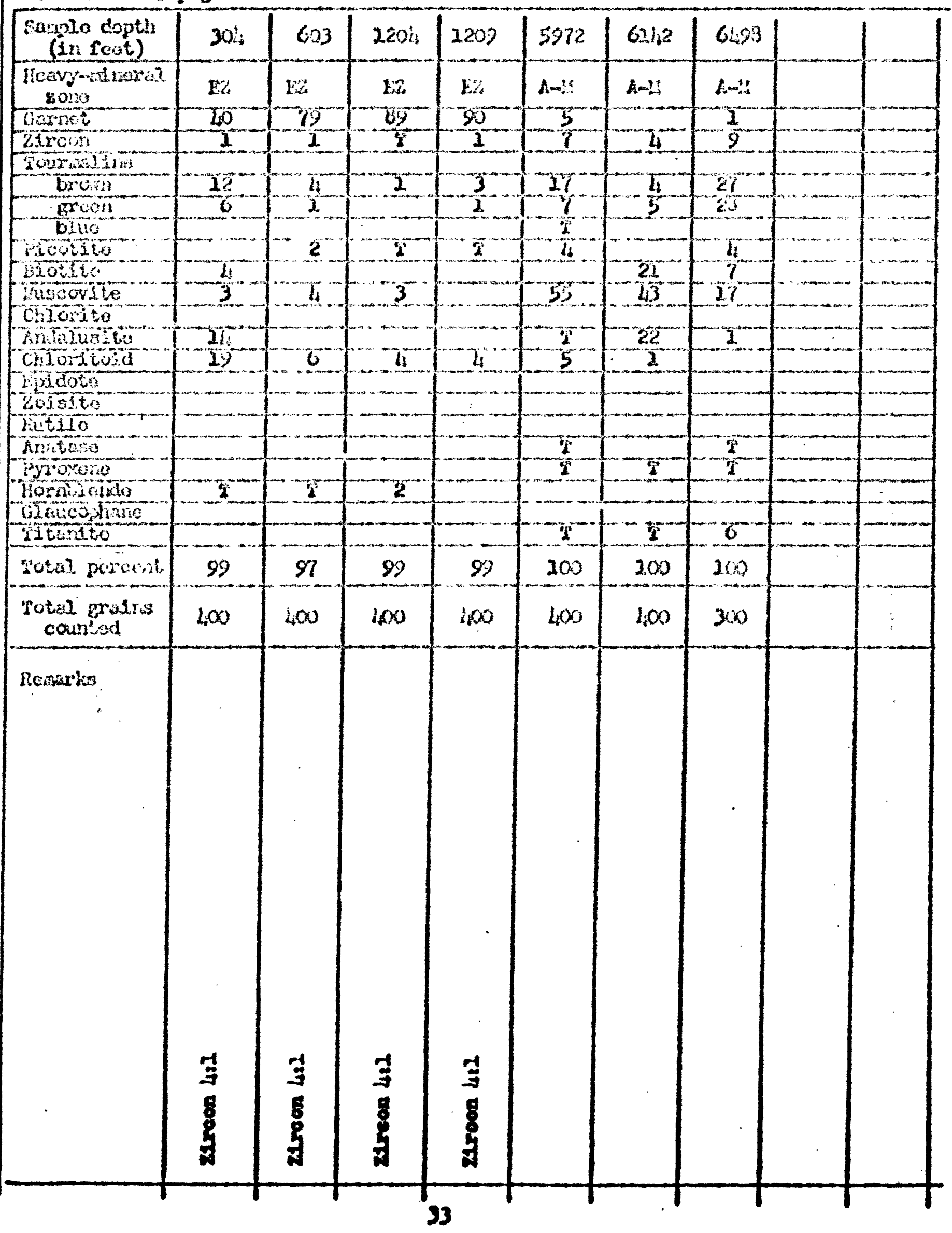


rable 2. Heavy witeral coayosition in trell seaples by percent

Fel1 krise - Undat jest Feil ko. 1

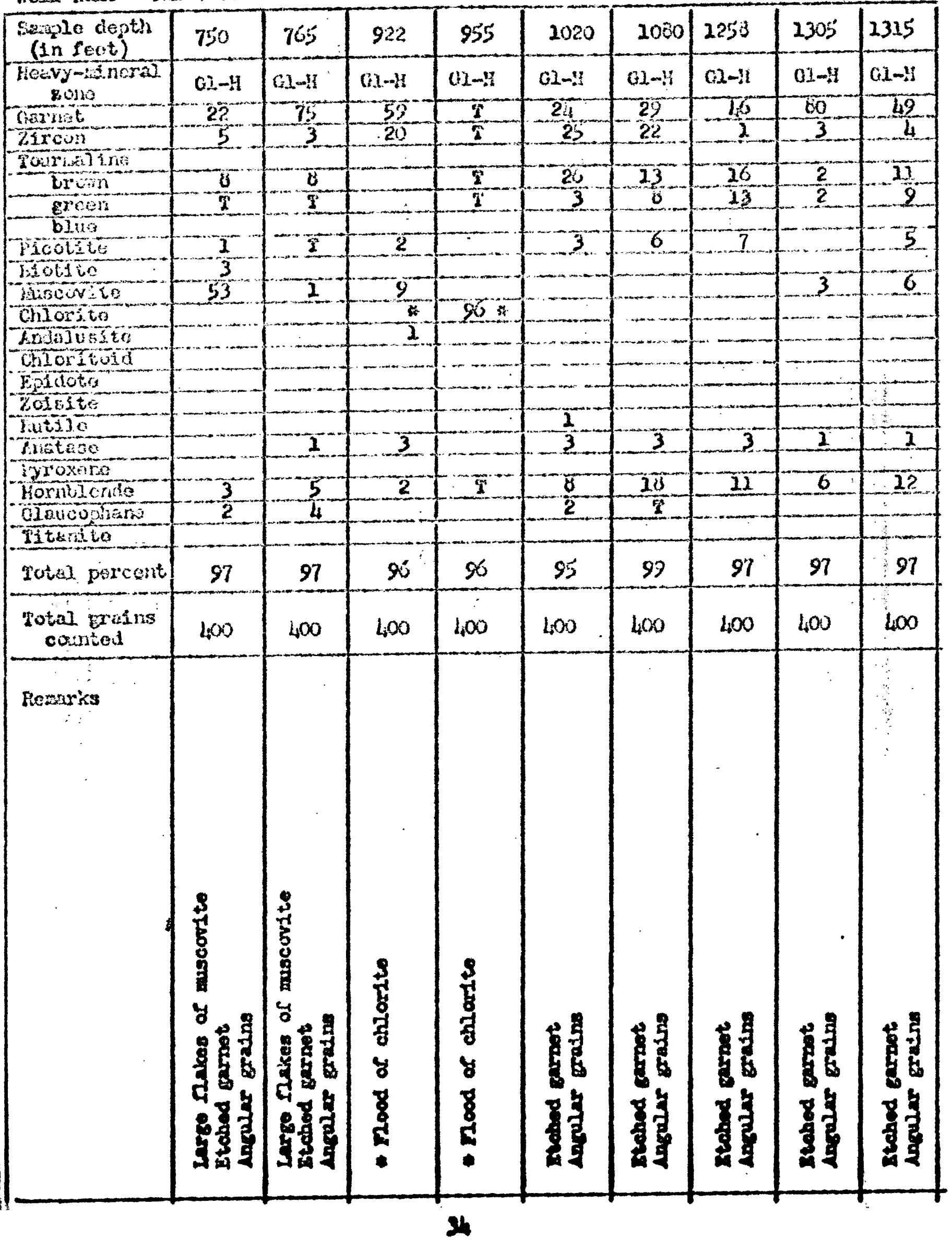


Tablo 2. Heavg at nural comozition in well sayjles by perosnt

Neil Veme - Uadat Test iell No. 2 (cunt'd)

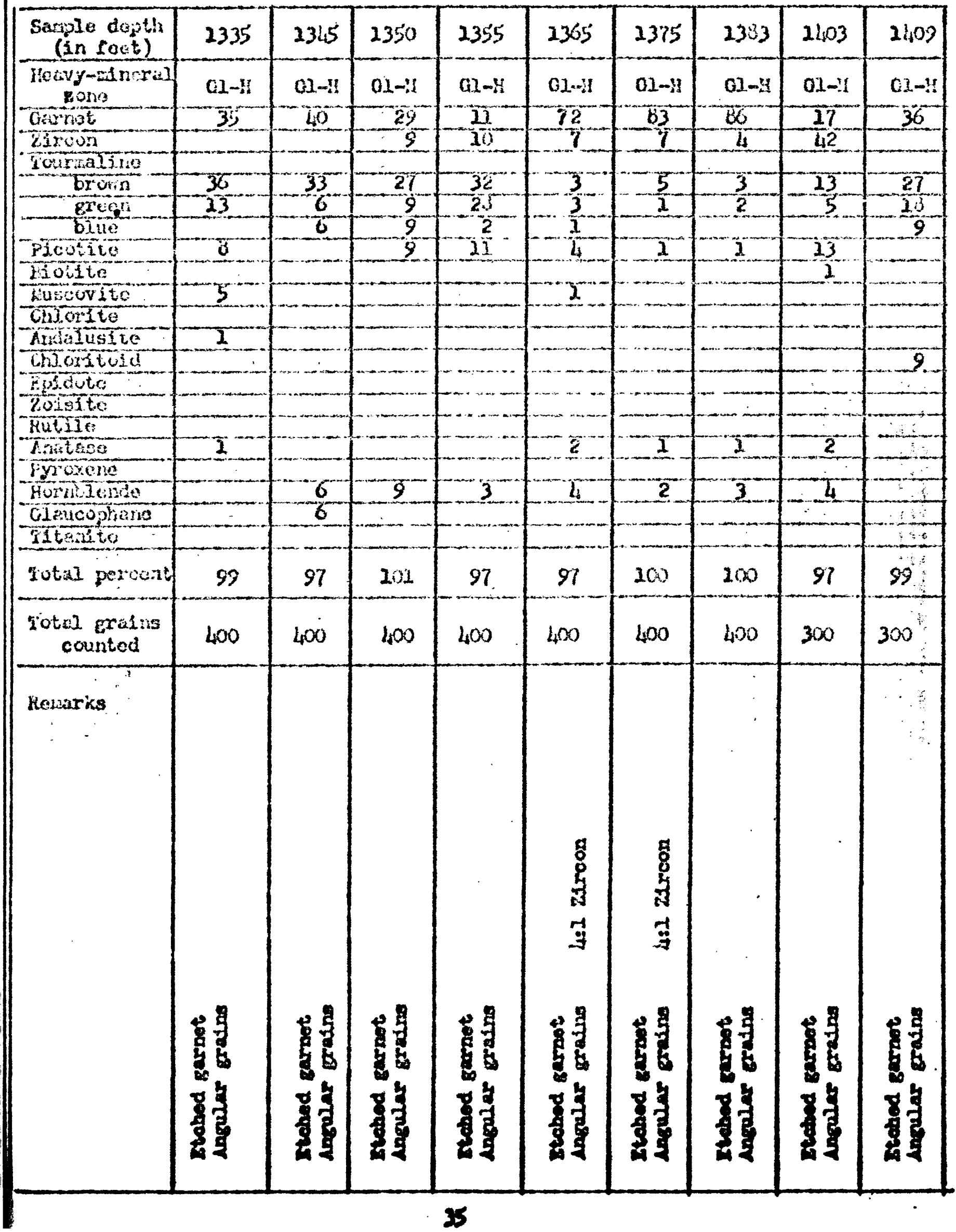


Teble 2. Heavy direral contonition in well sumples by percent

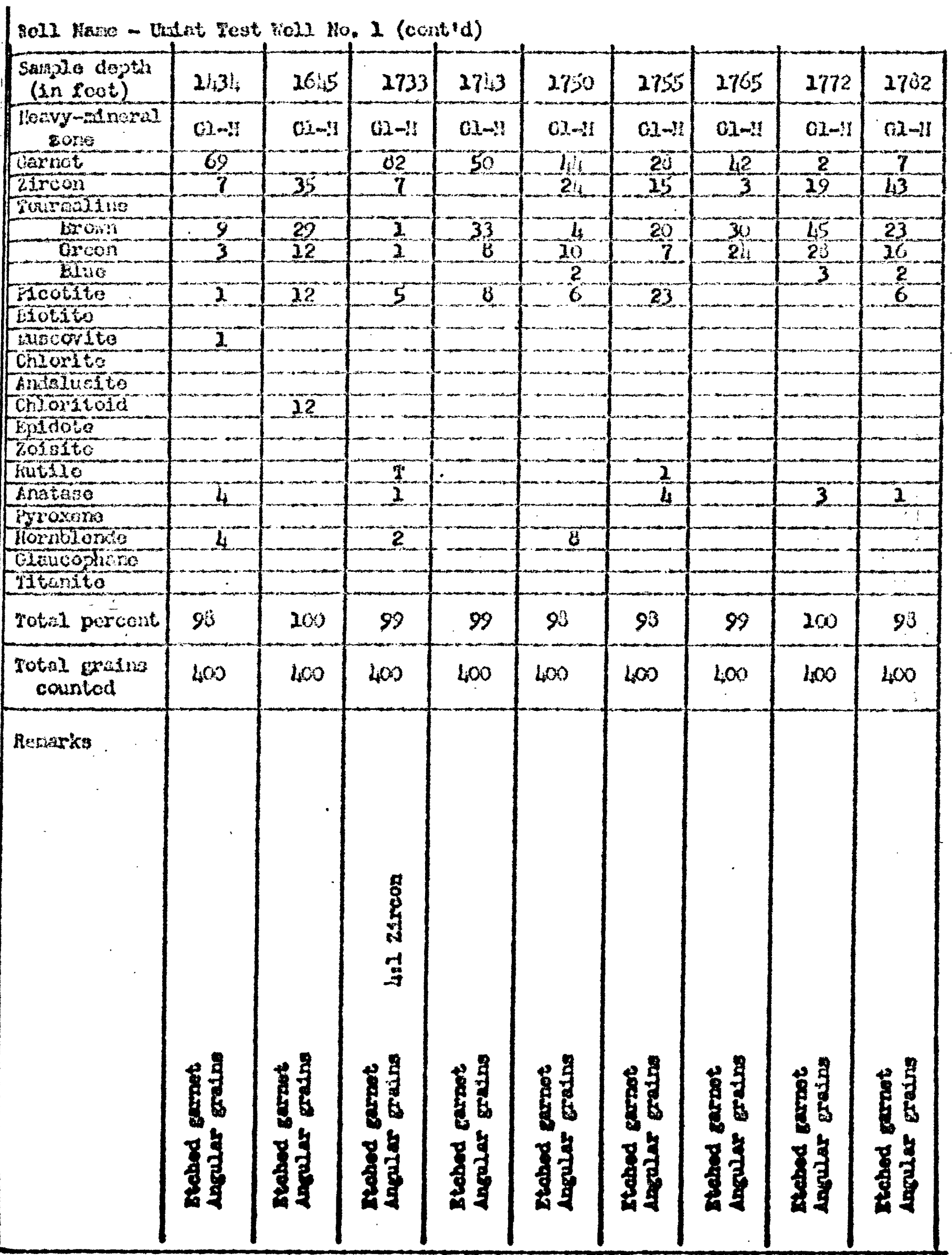


Table 2. Hoavy ineral coaposition in vell samples by porcent

Dell Nasa - Valat Tost Nell Ho. 2 (cont'd)

\begin{tabular}{|c|c|c|c|c|c|c|c|c|c|}
\hline $\begin{array}{l}\text { Satole depth } \\
\text { (1n fect) }\end{array}$ & 2792 & $2270^{\circ}$ & 232 & 2297 & 2302 & 2309 & 2314 & 2310 & 2323 \\
\hline $\begin{array}{l}\text { Heavy udnerat } \\
\text { zono }\end{array}$ & G2-11 & (il-14 & $01-11$ & $a x-1 \mid$ & Cis?! & $a x-i x$ & 01-n! & C1- -4 & $02-4$ \\
\hline Garhet & 4 & 70 & -95 & 83 & 93 & 90 & 90 & 05 & 60 \\
\hline 21 ron & 39 & -7 & $\frac{2}{2}$ & 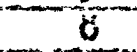 & 3 & & & & \\
\hline $20 u r a j t r e$ & & & & & & & & & \\
\hline brian & 25 & -2 & 2 & 2 & $I$ & & & & 2 \\
\hline Eron & 20 & & -2 & & & & 1 & & \\
\hline b2ue & 2 & & $\ldots$ & & $\ldots$ & & & & \\
\hline Picoute & 7 & 2 & 1 & & -2 & & 1 & & \\
\hline Wiot & & & & & & & & 1 & \\
\hline Hoovitto & an & & & & & & & & \\
\hline chlorits & - & & & & & & & & \\
\hline Anglueste & $-\infty .$. & & & & & & & & \\
\hline Dhostodd & $-\infty$ & & & & & & & & \\
\hline Budule & & & & & & & & & \\
\hline 2010168 & $-\infty$ & $m$ & & & & & & & \\
\hline 10010 & & & & & & & & $I$ & 2 \\
\hline $\operatorname{tratses}$ & -3 & 4 & & 2 & 2 & -3 & $I$ & 3 & \\
\hline Pyouenes & & & & & & & & & \\
\hline los rolonde & -5 & -32 & 1 & & 3 & & 7 & 5 & 12 \\
\hline Oleuco, 215 & Int & & & & & & & & \\
\hline iluats & & $\ldots$ & & & & & & & 5 \\
\hline Towel porocht & 99 & 102 & 100 & 99 & 102 & 99 & 100 & 97 & 93 \\
\hline $\begin{array}{l}\text { Tuted dajis } \\
\text { counted }\end{array}$ & 400 & $1 ; 00$ & 1,00 & 400 & 4,00 & $4 \times 0$ & 400 & 4.00 & 100 \\
\hline Fionarios & \multirow[b]{2}{*}{ 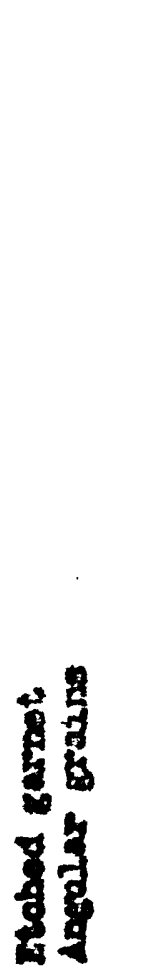 } & \multirow[b]{2}{*}{ 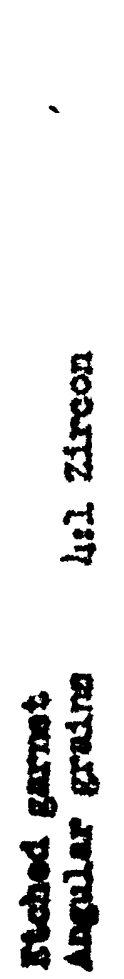 } & \multirow[b]{2}{*}{ 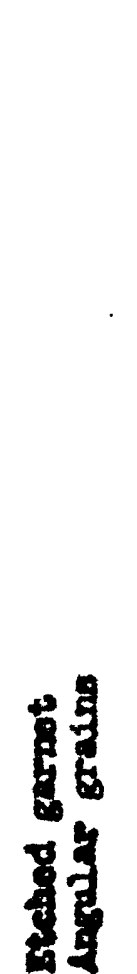 } & \multirow[b]{2}{*}{ 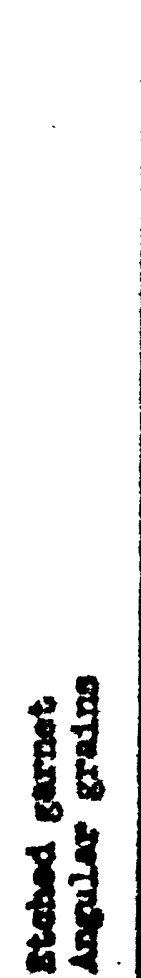 } & \multirow[b]{2}{*}{ 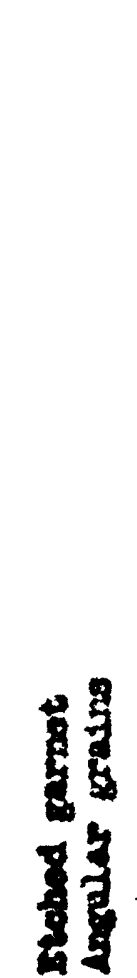 } & \multirow[b]{2}{*}{ 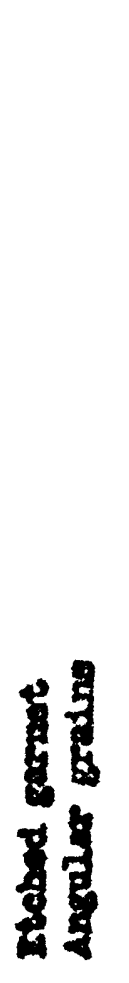 } & \multirow[b]{2}{*}{ 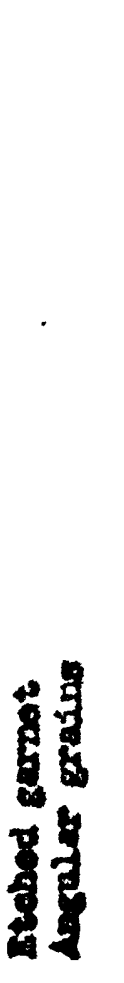 } & \multirow[b]{2}{*}{ 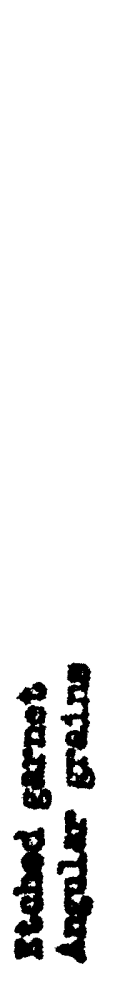 } & \multirow[b]{2}{*}{ 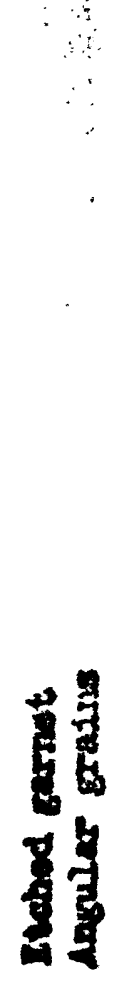 } \\
\hline & & & & & & & & & \\
\hline & & & & & & & & & \\
\hline
\end{tabular}


Tablo 2. Hotvy ulnoral conosition in kell sarules by porcent

Foll New - Unat Teat Houl No. 2 (cont'd)

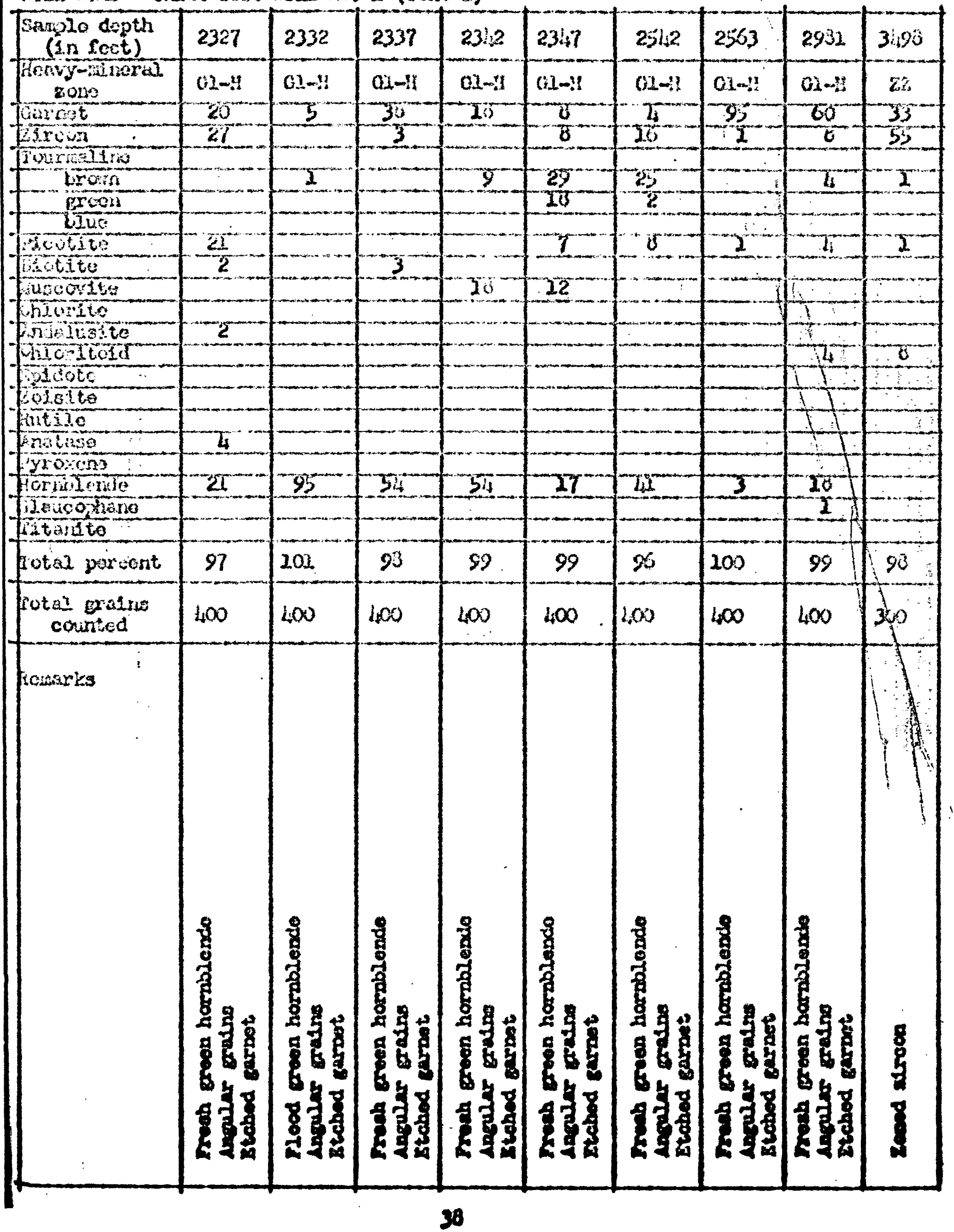




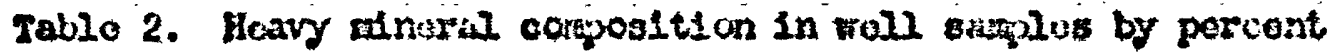

Foll rawe - thatst fost holl ho. 2 (cont'd)

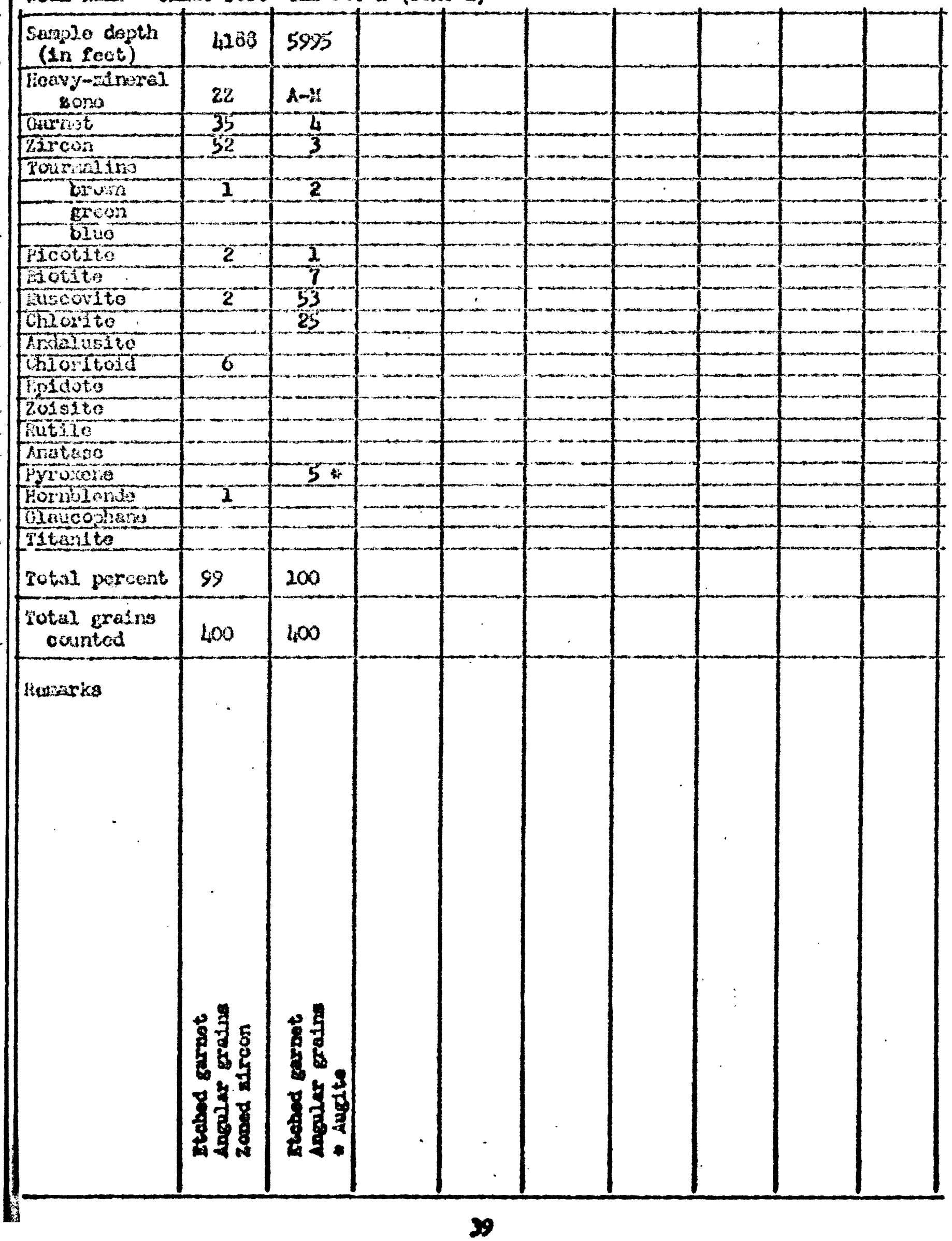


Fablo 2. Hoavy minoral coiposition in well angles by percent

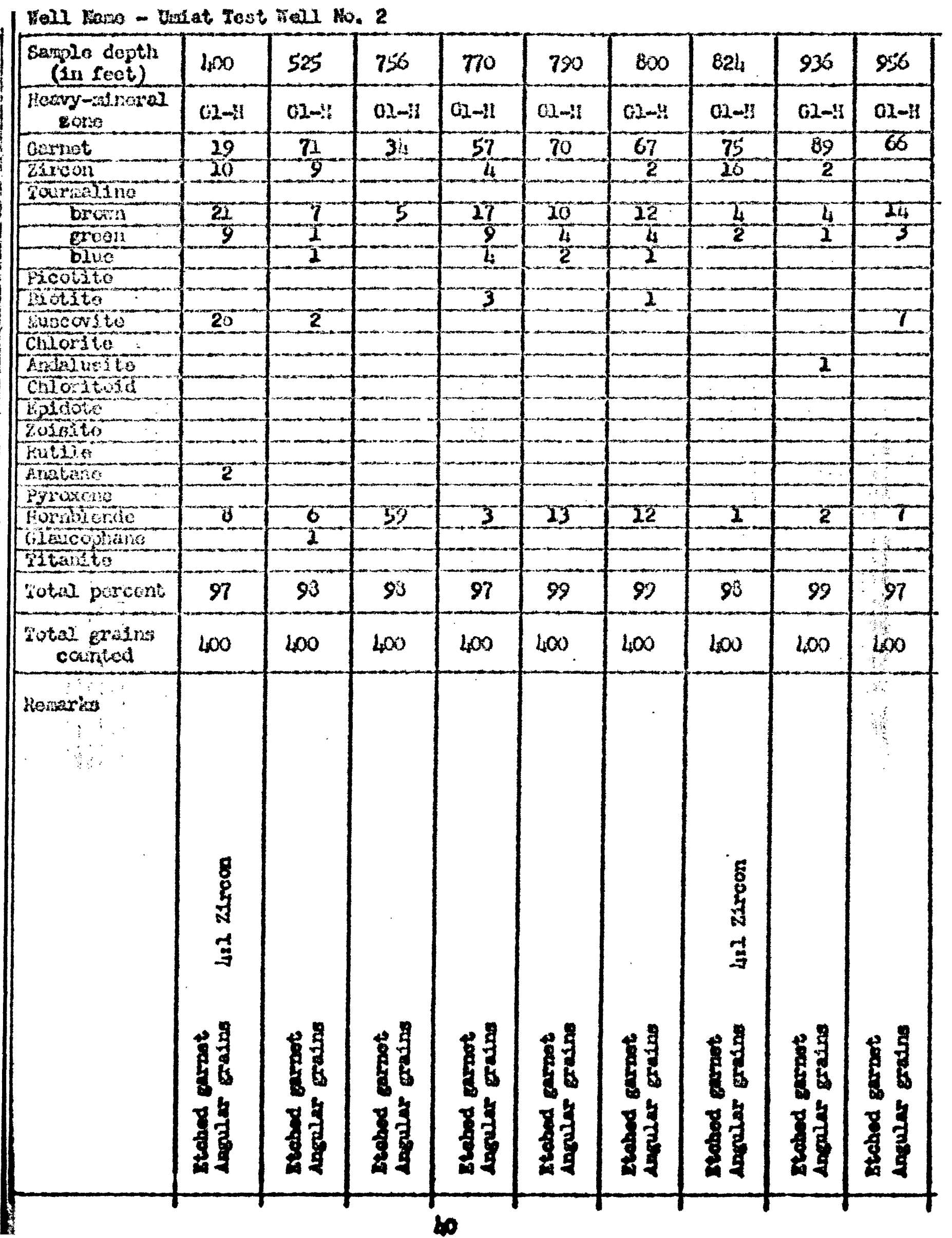


Jablo 2. Heavy noral conposition in well ofmplos by parcent

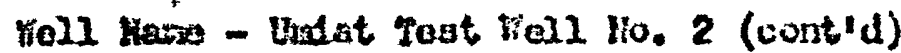

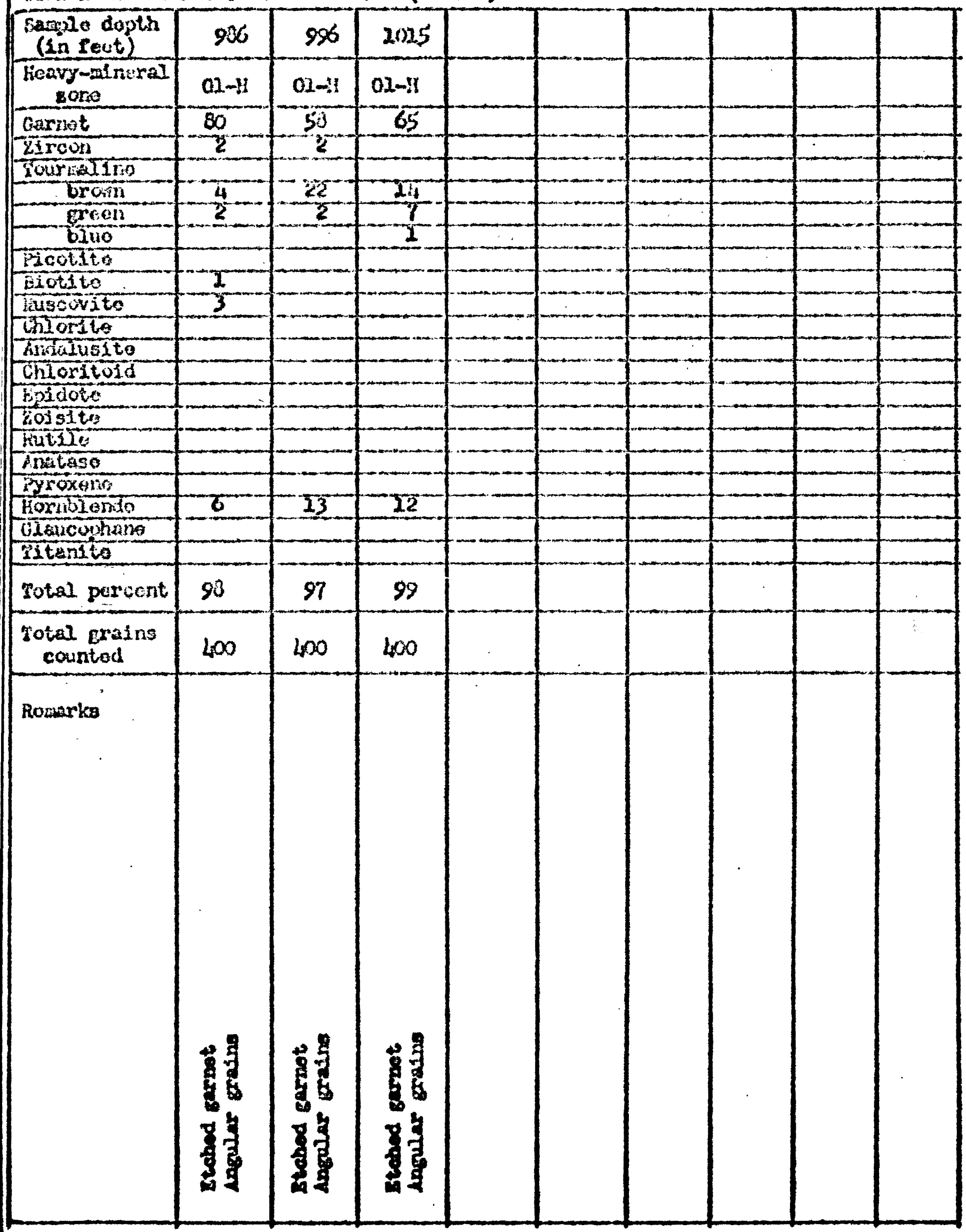




\section{Doscription of natratals}

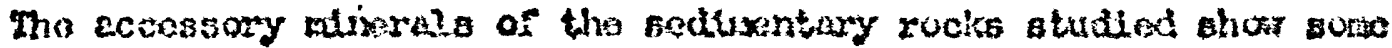
Intoresting minoralogic featuras. Son of theso foaturas aro used to dis-

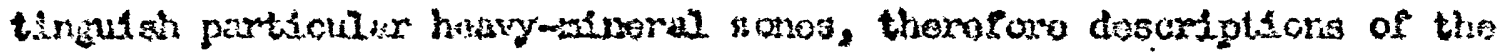
minerals aro given bolat.

\section{Dotrital opione efnerals}

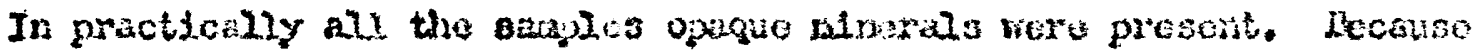

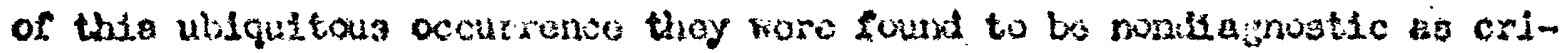

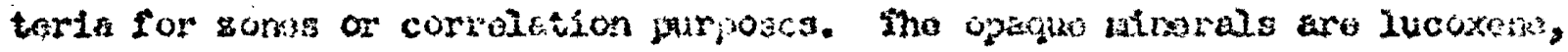
11noufte, and rignetite.

\section{Dérital nunopaque zíngalo}

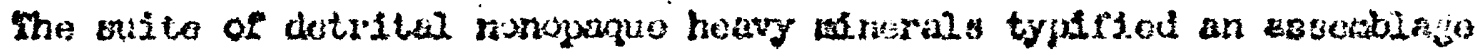

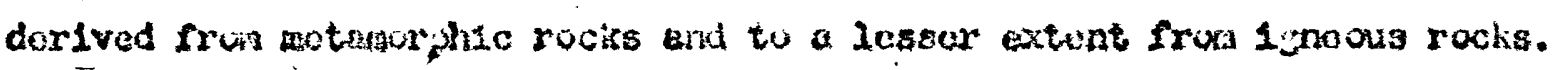

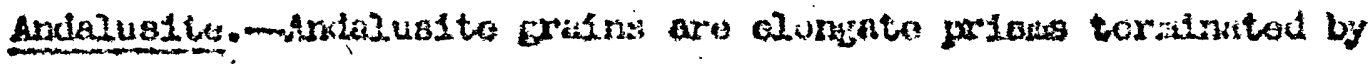

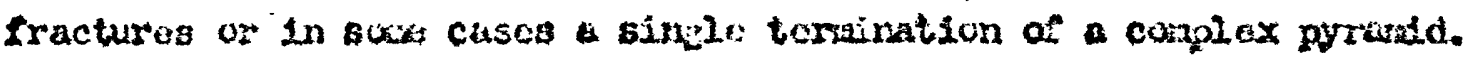
The erajus ro usually colorics3, nany havo carbunacous inclustons sonally arranich parallol to the crystellograthic $c$ axis.

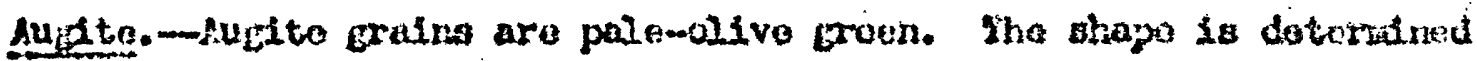
provarily by clcavaga. Socs of the grains have thin laths protruding from

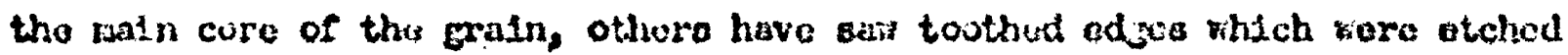
ait by 1ntrastratal solutions.

Biotite.-mo types of luotito aro pressont, 11,ht-rust-culorod variety and a dark-brom trangluoent virioty. Sho rubl-colured biotito groins are ouhodral to subhectral and contain pasoive (i) Inciusions. These greins are particularly abunlant in the bantonitic beds of tho 
seabee nomber, and thorofore bay bo interpretod es bolice a product of

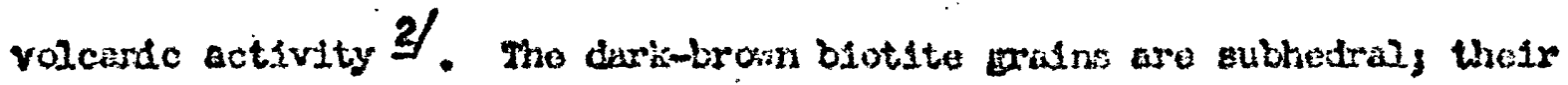

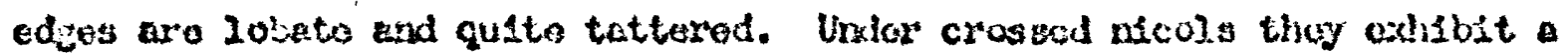
"birds-eyo mapla" appoaranco.

Chiorite.-Chlorito plates aro comion in a fen of tho capias. The platos aro 11 ht greon and havo 20 beta cojos. chloxits 13 not a charac-

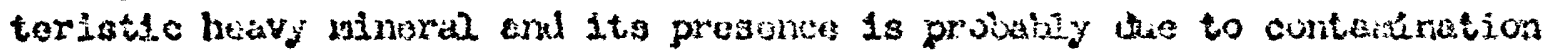
during tho ecparation of the hativy drestals.

Chloritodd. - Whoribold ocars es euhodral to buhliedral plats with

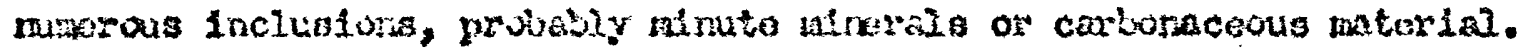

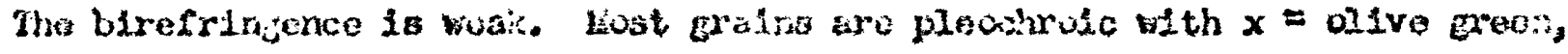
$y=$ palo blue, $z=?$ (duo to orlentation no color for the ray direction

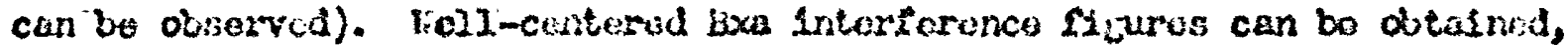
tho cptic eign is positive and the angle of $2 \mathrm{~V}$ is $10 \mathrm{tr}$. The relinf is very Migh.

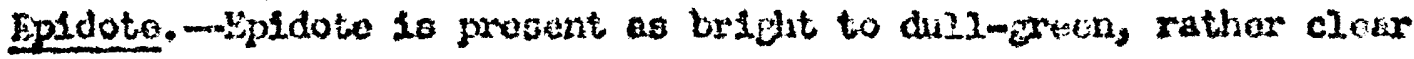
grajins. Cuntered optic axis intarforence figurea way be observed with acst grains, $2 V$ is 2 arize, and tho optic olgn nugativo.

Qarnot.-\$oth plnk and culorloss Earnot grains ero presont in Jurassic, crotacsous, and fuatornary rocks. Althouth cono grasna are noclurod, tho ourfices of nost ere ofched by intrabtratal solutions. Both varioties coen equally susceptibla to otching. Friangla rocks of the Earrom wolls ara barron of garnct; its absenco hare any bo attriluted in part to removal by action of the Intrustratal sulutiono. Buhodral graino aro prosont but vory raro in hoavy realcues frow the upjor part of the gopajoruk nomber.

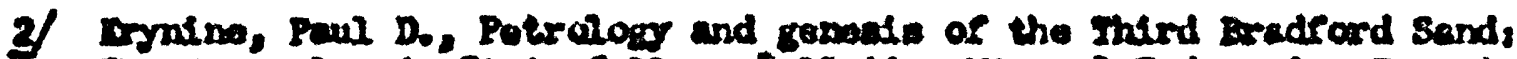

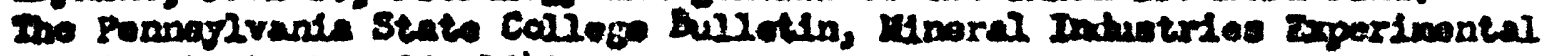
station bull. $10.29,2940$. 
Olaucophano.-Olaucpoliani occure as el ungate prisisa the shapo of

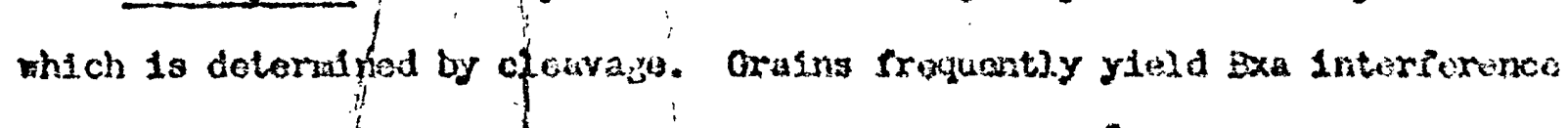
figures; the optic aign fo nigative with 24 abuit $40^{\circ}$. 2 is inclined to c plus $4^{\circ}-6^{\circ}$. Tho ninger is pleochroto with $x=$ coloxiess, $y=$ violet,

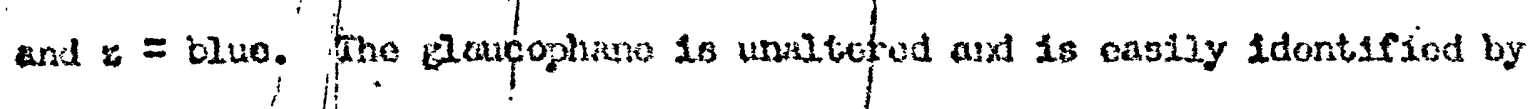
1ts rom and plechros ses.

Hornblendo. - Tromblande occuras in fro varietles, "cuation horntiende"

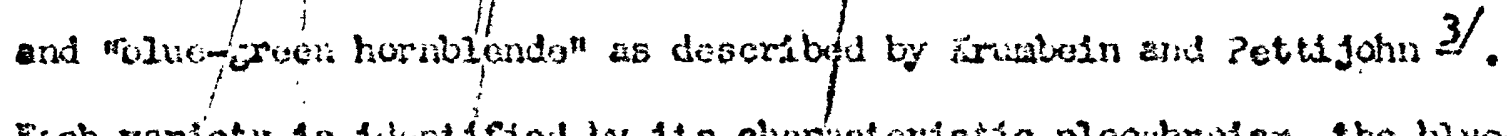
Fach varioty 18 flentified ly lis chaructoriatic pleokrolas, the blue-

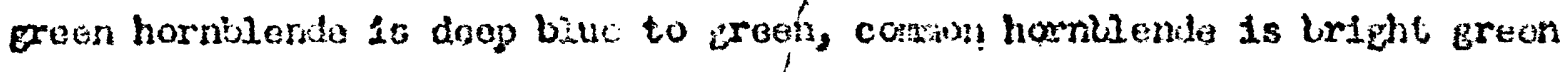

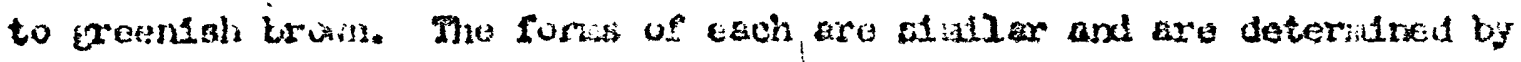

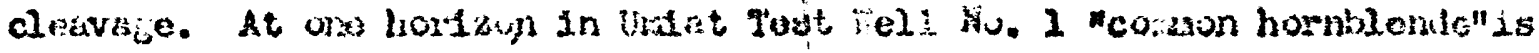

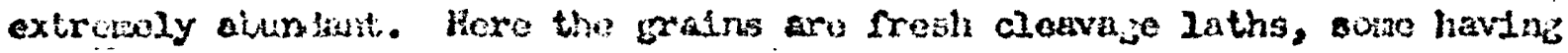

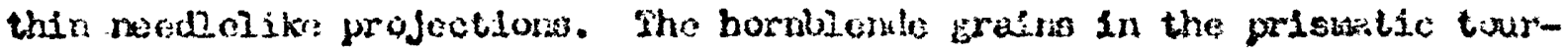

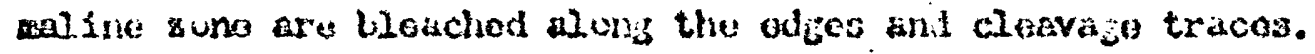

Luscovite. - Suscovite occurs as thin cletwage nakes vihich yiold good Bxa interferonce figures rith negative oytse sign. The edges are lchate and worn. Nuscovite has a specific gravity hisch is alightiy lees than

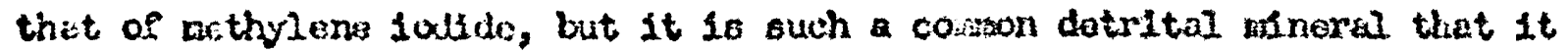
Ireguentiy makes up conoliferabio part of the heavy resicha: Ita prosence In the heavy portion io probally des to consnidnation. Plcotitu. - . - Icotits, the brom spinel, is distimguiahod by Its deop bean color, lactropian, and vary hij relfer in Canada balsars or in

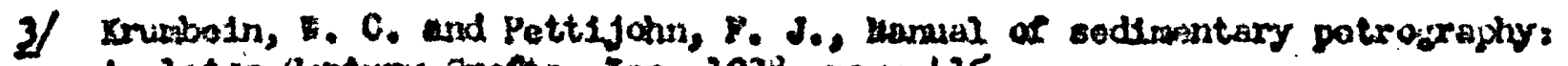
Apgletion-Contury-Crorta, Inc. 2933, page 425. 
aroclor. The pratns aro ainust opeque in the thick central parts but are translucent on tho thlunor edges. All grains ar't marized by concholdal fracture3. A fex graina are greongh brom.

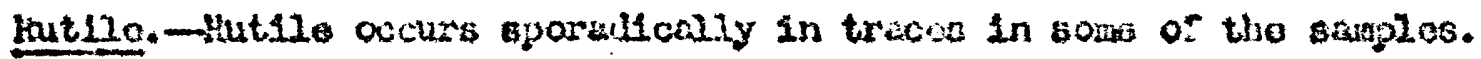
The grains are goneraliy elongeto duep-roddish-brows subinedri. The ralinf

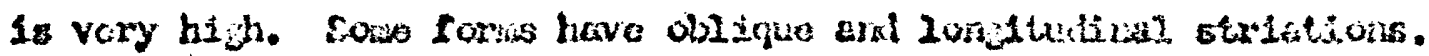

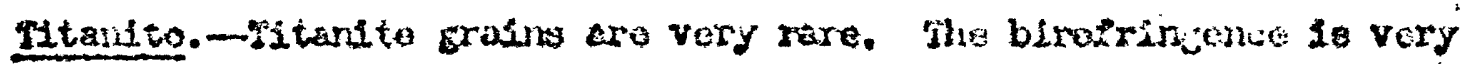

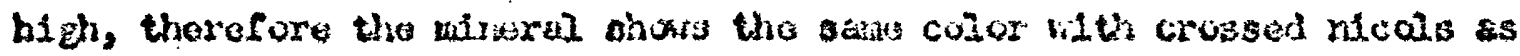

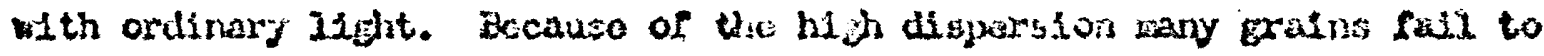
extinguish with crussod riteols and white 21 ht.

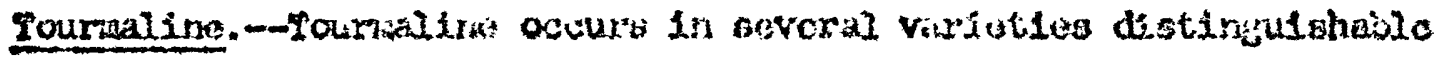
by color and dichrolam. The varietion can seldon be classirich as to opscies except indicuifto, which is blue. Tho several color varioties are

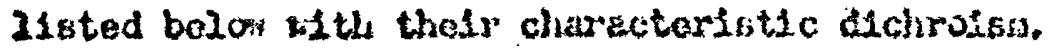

\begin{tabular}{|c|c|}
\hline E riy & 0 xisy \\
\hline $\begin{array}{l}\text { 21;int brom } \\
\text { 21ght green } \\
\text { maviv } \\
\text { doep bluo }\end{array}$ & $\begin{array}{l}\text { bromi (dravita ?) } \\
\text { olive green } \\
\text { olivo green } \\
\text { biue black (indicolite) }\end{array}$ \\
\hline
\end{tabular}

In the tables tho rave-aldve geven and the oreon-ulivo oron varleties are tatulated tojethur. In the triessic sandstonis and tho Oubik fornation olive-zrean and krorn tournolino wains are kell-zounded; in wh other sandstonis thoy are prismatic with pyrand tarainations on one end and cross Eractures on the othor. The blue gratns aro ccruholdaily sractured nat chlpe uaually orlentod parallel to (001). These nat blue grains

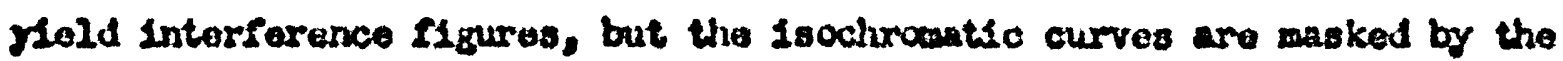

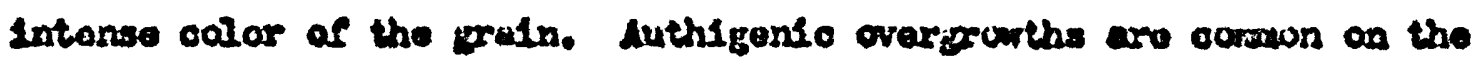


bromin end olfre-areon exalno. Sore orcins have orold cores which have beon secondarliy cllarged. Tho coros aro not aimby the same color ur

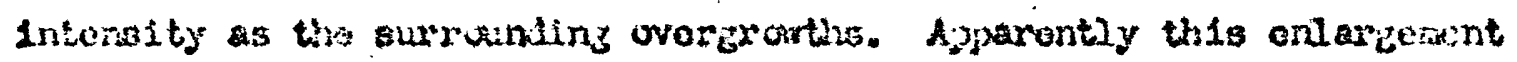
occurrod betore the grains wero erudat and incorporated in tho rooks from whach the earjatis wore taken.

Zircon. - Several types of zircon Existas aro prisent and are uscful

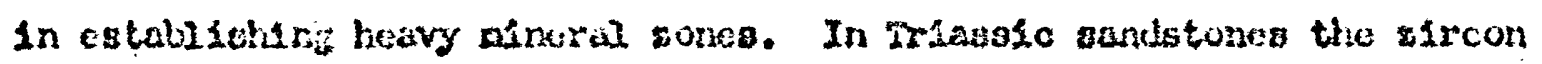

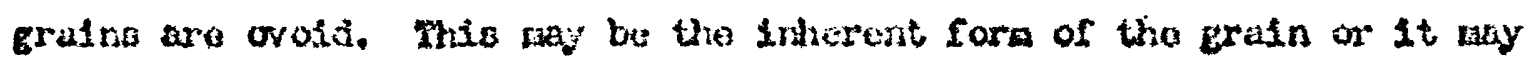
bo due to rourding during trensportation. In tha uplor part of the rorok forsation, the ruktu waber and the 20 an part of tho ropajoruk maber many ziroon crjotels are zured. The prestance of these gonod crystels is the besis for estabishing the zond gircun pone. Such zurod alrcon crobtais oro 11 ght yellos or 21ght pink ard hive lonith to vidith ration of about 3:2. In tho upper part of tho ropaturuk wanber long olender culor2038 zircon crystals with first onlor prisias and promdin are the characteristle fora. Tho lenth to radth ratios of these colorless crystals exceed 4:2. Thoy froguently have acicular $21 q u i d(1)$ or arseous (3) Inclusions. stuby coloriess and pink zircon cryatels we alsa prosort throughout most of the Cretaceous bandstones.

duthigenio heuvy whorala

Anatase. - Anataso occurs as 21 ght-yollar guthidral tabular grains. Host aralns are marked by gooxotric pattorns. The interferanco figure 1s unisaxial negativa. Whothor or not this mitnaral is authigenie is difficult to dateriatno. Tho associated oratns are exeah frecturod frazwants with no obvious roundine or wear un the diges; therefoce, if the 
anatase grains are dotrital, no ovidence of vear phould ba expocted ard tho erains showld havo dietinct borlors. However if the Exafis are

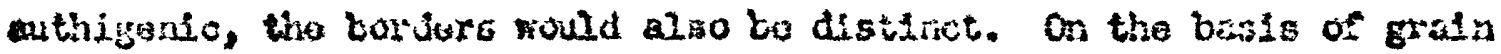

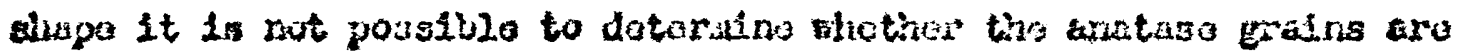
authiganic or detrital.

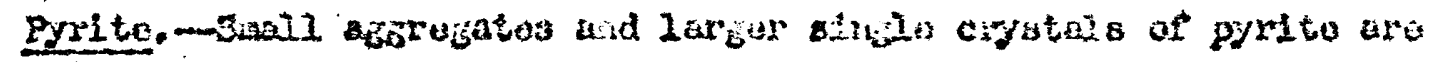

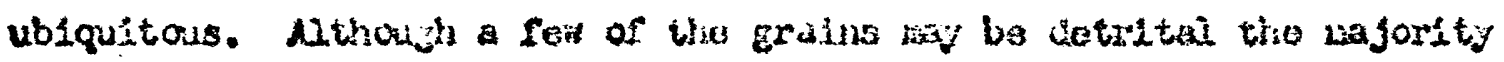
aro athiganic.

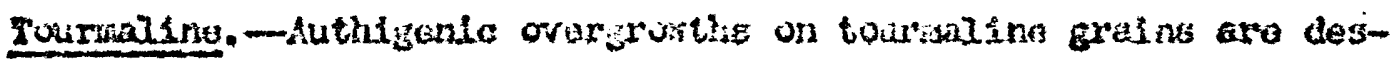
cribod un page 4.5 .

Meary-aineral goitos

Recional sonation

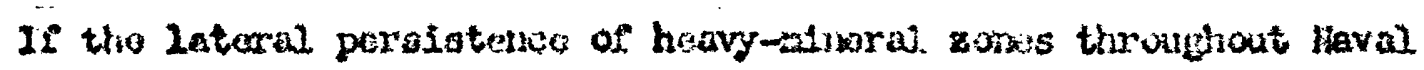

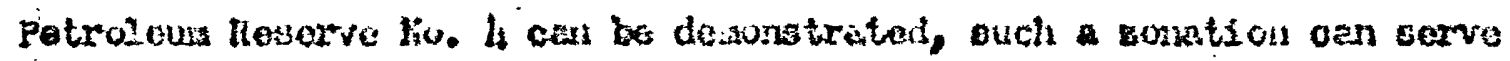
as a uaful corialution dovice, heavy-atnoral sonation has boun estrib2lahed and corralations mado by using tho fulzowige critcorias (1) pre-

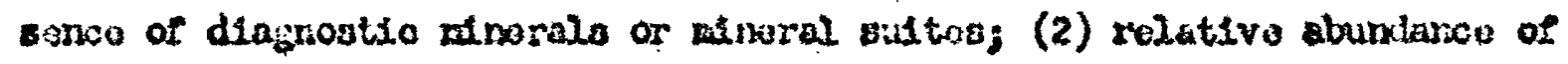

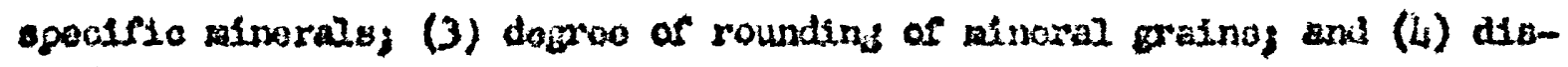
sinction as to wain ford, whoh as ouhedril sircon cryotals with a longth to width rat10 of 4:2. Blyth Hstinot zoned cais bo rocogntred: roukied

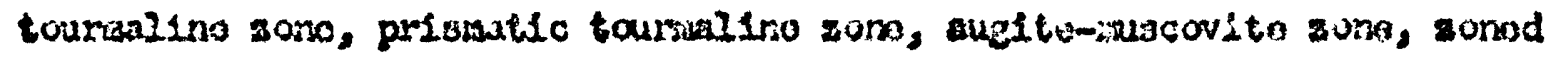

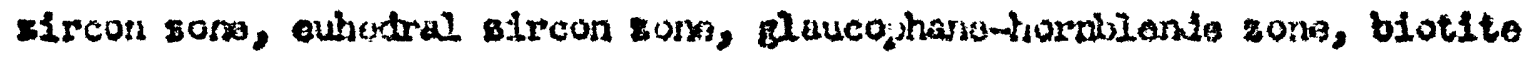
sone, and the ruanded erain sono. Descriptions and charactariatics of the aneral sanes are drun bolar. 


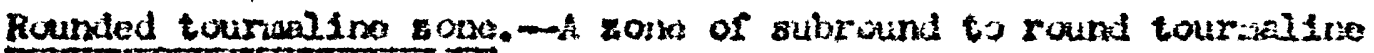

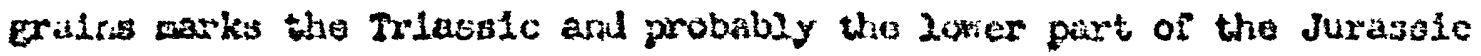

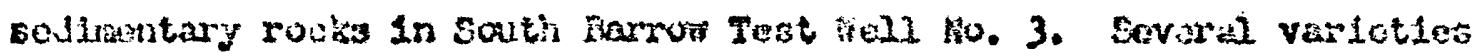

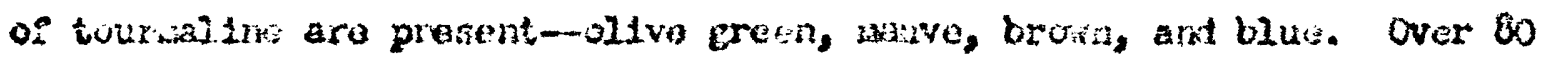

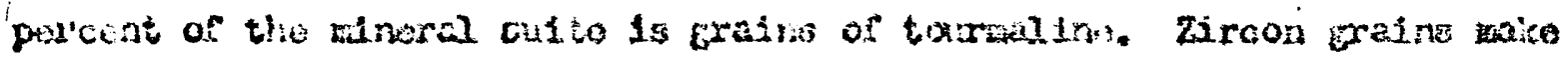

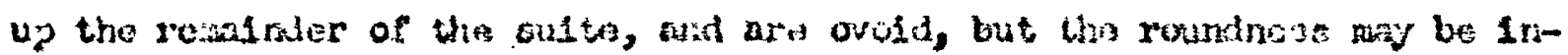

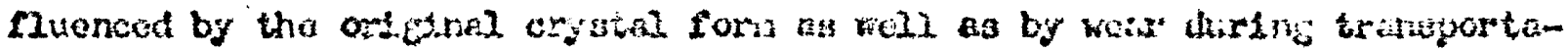
ttor.

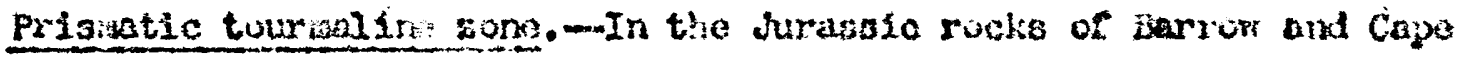

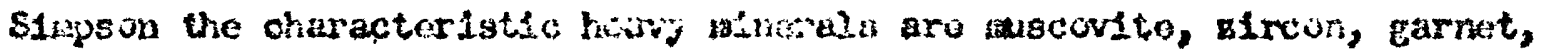

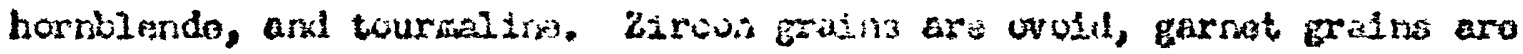

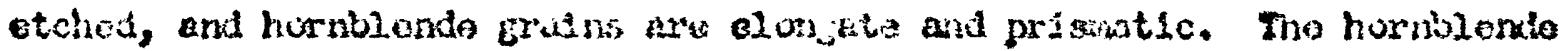

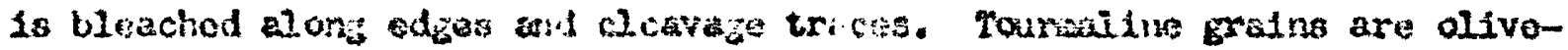

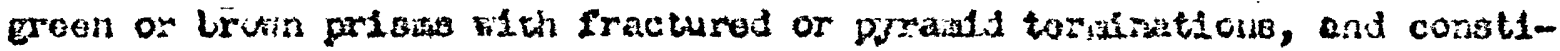

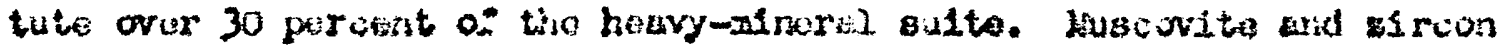
Exins are abundant. Hombionle and gaint Eraina aro rare.

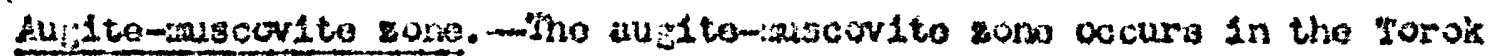

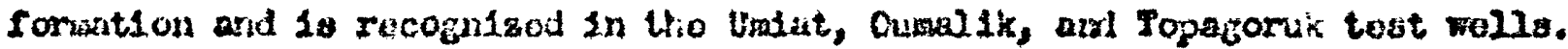
Luscovite prains aro particularly abundant, fircon and touraline grosins aro

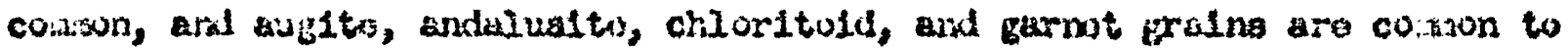
rare. Evon thou;h augito grains aro raro in soso saryles, thoir presenco is

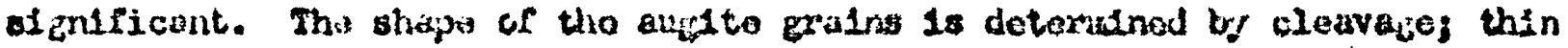
doltcato 2athe nhich havo saffered 2ittle cbrasion during transportation protrude frua suan of the gratns. Intra otratal aslutions havo otohed a few

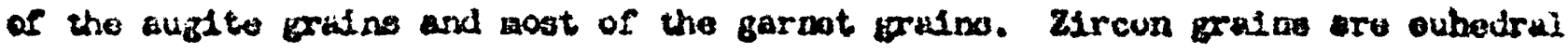
and hava a leagth to width ration of about 2.5.1. The touranisu arane are prosiant10. 


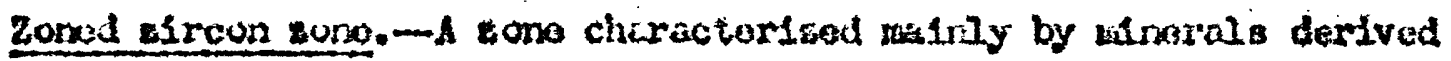

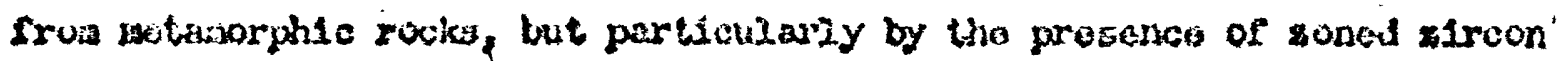
cryotilis, is prosont in part of tho porok forsation in the Barrusi area,

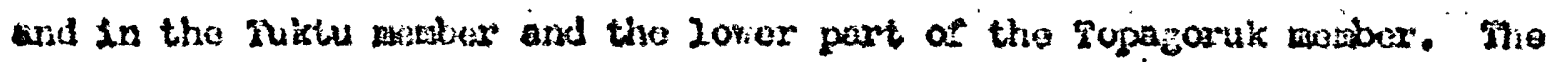
soned zircon grains are unuejly dombly terwinated, hava a length to dith ratio of 3:1, and aro yejior or varz 2 jugt pink. ovold pink und calor-

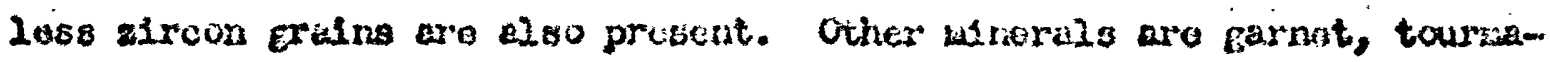
2ino, picotite, biotite, endalusite, and wascovite. A fort touranines

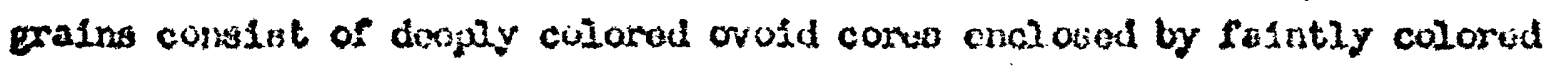
ovoryurthe with pribisitio faced. The corn and the ovarisorth of an . Individual grain tay bo the sa:n culor ur diriterent colors. Many garnet eratno are otchod. Other wincral erains aro aubhodral or angular.

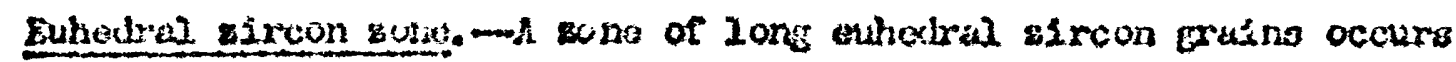

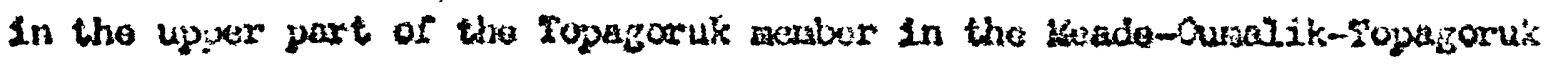
area. The ewtidral sircon wain aro culorless aud have longth to width ratlos which axceed hil; first order prisns and pyraratio aro cosinon. Soso plink sirculi crasis are presont and they have nora couplax cryotel Ioras. Oarnet; tourasidris, biotito, bascovito, andalusito, picotite;

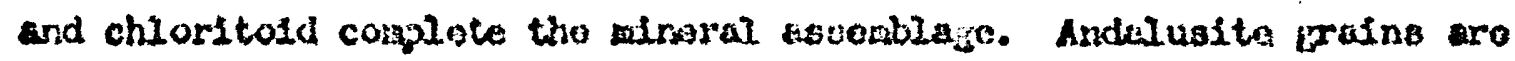
prisantic, with singlo terulnations of conglex pyrieside, and ary contaln carbunacoul indusiuns oriented parallel to the axio. Fine bion tite plates are culored doep bruin or 21 iht-rust brumn.

Olaucophano-hornblerdo sone. - The base or the elmicopheno-harnblondo cono is defined by tho proatest depth at which plaucophane is oncountered $(2,981$ feet in ondat Ieat noll Ho. 1). The tog of the suno 28 marked by 
the base of the blotite zoro described bulow. The sone is present in the

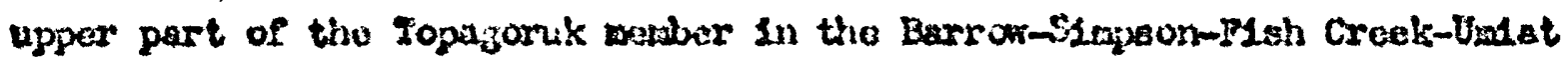
area. The elaucuphno-hormblende suno and the oihodral sircon zuno aro therefore Btratigraphic equivalents. The otnoral sufto of this zone includos gornet, touranine, eircon, blotito, auscovito, andalusito, horn-

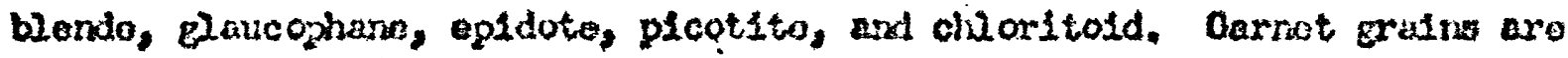
pink or colorless ary a majority of thea are otched. Maucoghano grains aro bluo to lavonder plrodirole laths with gool cleavago. Tho hornbleado laths aro pleochrulc doep blue-grean to green end aro rare to cuiron. Soth deap brown ard 21 ghimrist bxam plates of biotito are conson to abumdant but never conglitute sore than 50 porcent of the wnoril sulte. Biotito sone- - In tho Capa simpon area a zone containing daris and 21 ght-rust brom biotite flakes is present in the Seabeo mater. The biotito nlakes ar oubhodral to euhodral and prominant in the bontonitic beds of this andor, constituting over 90 paroent of the heary-ininaral esserablago. Tho averego thiokness ruprestanted by this zone is 200 foot. tho subordinato minerals are glaucophawe, fircon, touratina, ondelusite, auscovite, and chloritotd.

llounded prafn sono. - A iono charactorized by wal-rounted grains of andaluaito, garnot, elaucopharo, cpldote, chioritold, blotste, muscovite, horntlende, tourwalina, and sircon is cluaracterisitc of the oubik forwation. The hizh detroe of rounting of the grasnis and the number of the anoral spocies prosent aro tho criteria for tho differontiation rou all

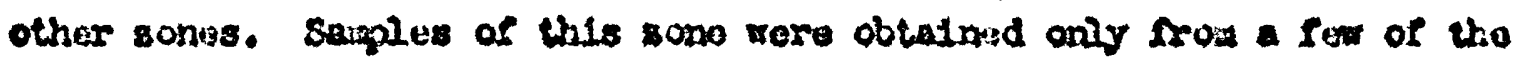
Bimpaon coro tests.

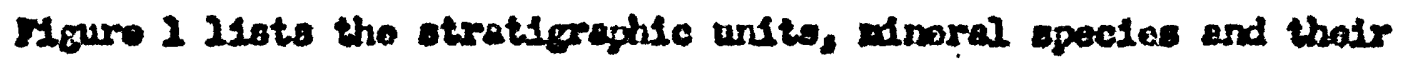
poralatence, and the raoges of the boery-abroral socos. 


\section{CORRELATIOS ON HEAVI-IIREML ZOKES}

Regtonal heavy-utnoral corrulations can be wde shen heavy-aineral zones pergist lateribly throughout a sedteanting basin. The persistonce of sunes afthin the basin is dapandent upon the wource rocks and their areal extent, veathering, transpartition, deposition, rackes pattorsis,

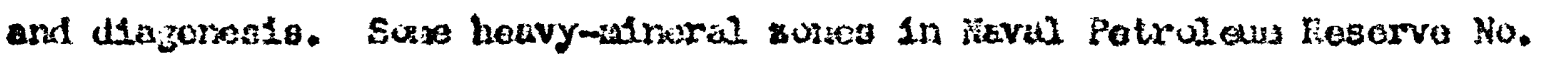
4 aro lateraly extmsive, othors aro knom only in local arwas (priatily because equivalont roce units hivo nut beon sajpled in surrounding aross).

The roumded turimalina gune 18 present in South Barrow gest fioli No. 3 betrien 2,430 and 2,690 reot of depth. Ho other welle porsetrate Irlassic

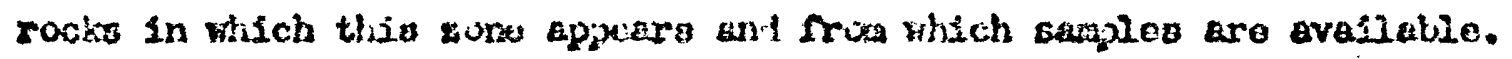

The prigatic tourtalline gone occura in South Darron Tost Fell Hic. 2

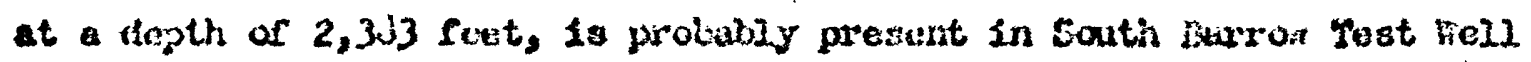
Ho. 3 boticen 2,200 and 2,300 feet (no suagles vero taken in this intorval), and is rejresentod by one sugle in 3tupson Test lioll Ho. 1 at 6,173 feot. Ho other wells havo punotratiod oquivalent rooks.

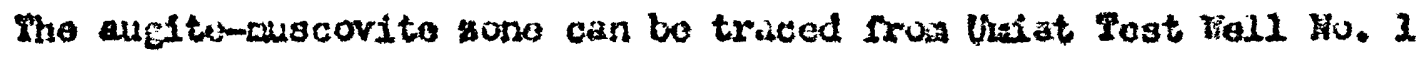
at a depth of 5,995 foet to Cuaralir Test rell No. I botweon tho depths of 5,972 and 6,1993 fout. The fuil vertical zlaits of the zune way bo areater than tloso statsd, es thoso litults are contrullod by the avaliablo cerplos. The northward thindins ant gradual pirching out of tho beds in which this 8one occurs, wo representwd in the section from Ousalik to Topajomik, is probably the ruason for the absence of the zorks in Simpson lost riell No. 1 and tho tarror kallo. 
The nost extensive some 1 s the soned sircon sono; it is reprosented In all but one of the deog test relle. The depths in the varlous wells In whtch the sona occurs are tabwated belok.

Vano

South barron Fest fioll Ho. 2

South Iarroes Tost hell Ho. 2

Bouth zarrof Test liail No. 3

S1apison Test Fall Ho. 1

simpeon care roat Ho. 23

Fish creak Teat fiels ko. 1

Uniat Tast Wall Ho. $I$

Cunalik rest liall Ho. 2

Headis Test Vell Ho. 2
Dopth(i) in feot

$2,907-3,3,30$

$2,299-2,330$

$2,405-?$

2,360-?

2,502-?

$5,530-6,009$

$3,4,23-4,103$

$2,151,-3,75 ;$

8-4, 123

The sono ohould also bo present in Topagoruk Tost Fell ko. 2 botreen ?,000 and 4,000 feot, but no earyl es aro avallable within this inter-

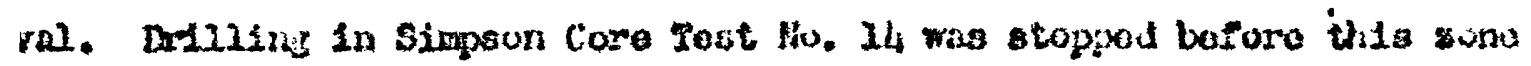

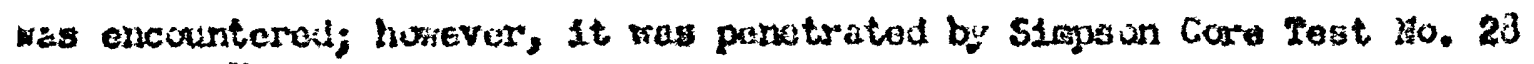
at depth of 2,502 foet. In ander that riguro 2 be wore corglete, the soction fron 2,502 feot in finpon Core Tost 30,20 is projected to Slapson Core Test No. 24.

Two zunes are strats graphic equivalents - the cahodral zircon zono

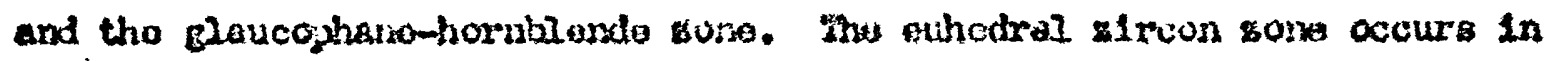

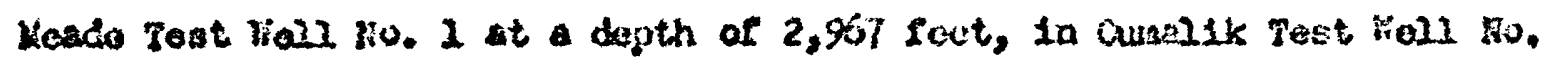

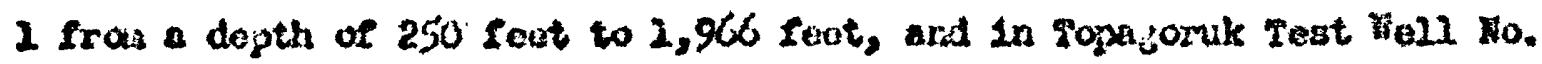
1 froa a depth of 304 foot to 1,209 feot. Forthrerd and castiand the sone

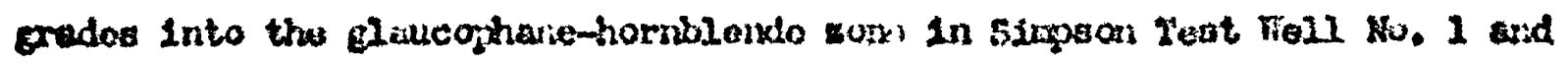
thatat tost tall Hu. 1 respoctivaly. The glaucopharie-hornblendo zund 1 a presont in South Barrow Just Fell Ho. 1 at a depth of 165 feot, in stapson Test Fell Io. I rrou depth of 230 foot to 2,297 foot, in 31mpoun Core 
Fost 10. 24 frow a depth of 320 feet to 2,213 tent, in Fish Creck Fest Dell to. 2 fros 2,920 to 3,030 foet, and in thaist rost foll to. 1 frua 750 to 2,981 fout. The mineralogical difierences leticen these tro sones aro attributad to difforences in source rooks and their contribution

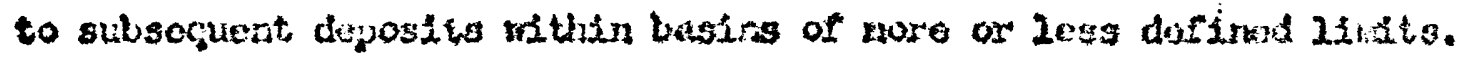

The biutite nono has been ldontified only in tho Cepo sirepsuil arua and 2 discussod in the soction dualing witi lacal corraiations.

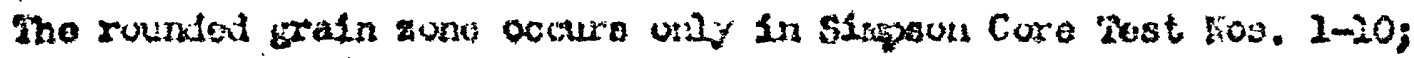
horovor, thes Gubtix foriation, in which this zura ayyears, covera atch of the Arotic constal plain. Saryliez fruat other welly woro nut taken. In

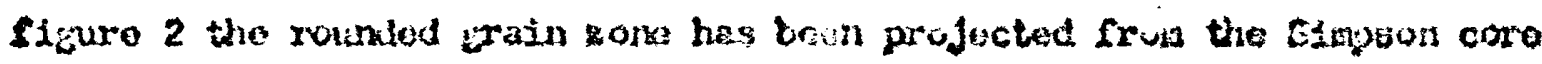

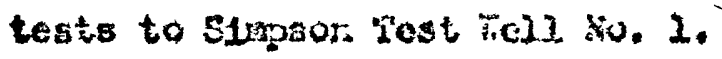

Flaure 2 illustratos the reational distribution kat currolations of thesu heavy-iafmaral cosws.

\section{Local distrilution and corrulation}

In 20032 arcas carrulations asong wolla can bo wode by uaing the

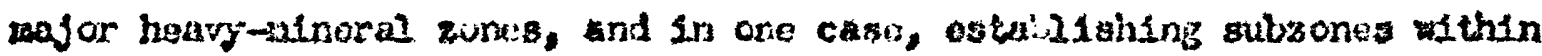

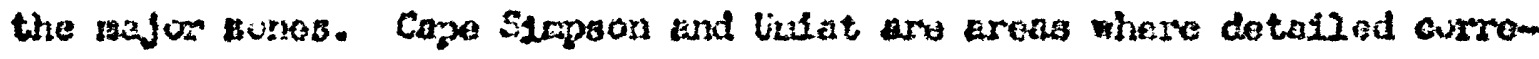
Lations ore poselbie.

\section{Cape Simpoun arua}

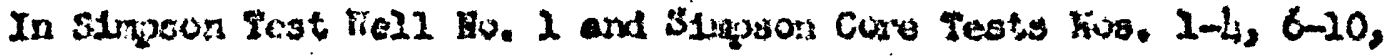
the recomisol ainaral zonos are those of the rounted prain wus and the

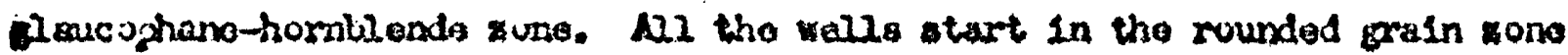

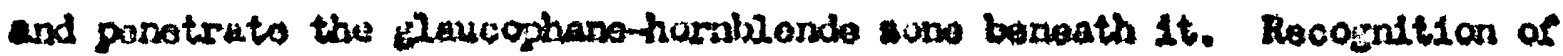
this contact, based on the degree of rounting, is strollo but earvos only 
to defino the base of the rounded gridn gone (Cubik foriation). Lack of equivalent stratigralic sarples precludes tho possibsility of a nore dotajled correlation within the gluucophare-iornblende Eune. Tho heavy-aineral sonation of these holas is chom in figuro 3.

Correlation of simpson core Fesis fius. 13-27, 23-26, and 23 is possibia usine the biotite wore and tho gleucopham-hosnblende zone, as shom in fizure 4. The cuntact dofined by these two zones watis the unconforutity betwoen wio upyor pirt of tho Tojajoruk maber and tha bess of the ceaboe nesbor.

\section{Uxiat area}

Subzones estatishod within the major heavy-idmal zones are ured to make dotailed correlations in tie tro baiat test vella. Table 3 has boen construcied so that the sagles usod for correlation are placed ojpesite

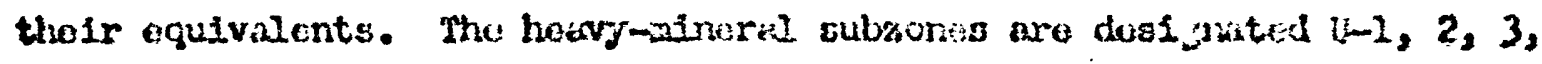

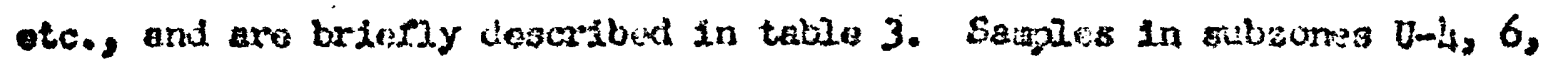
and 8 proved useful in correlating the tro rells. Sayplos fruid d2is feet

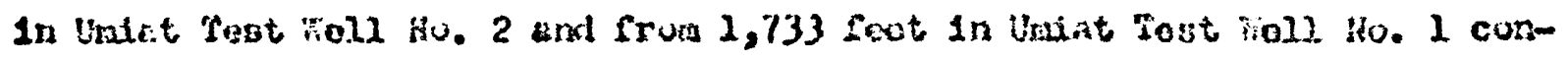
toln 75-33 porcent garnit, 7-16 percent sircon (12ugth to wath ratio, 4:1), 4-9 parcent trom touriallik, 2-3 percont exeen tourialinw, and 1-2 porcent hornblenda. Satgles at 525 feet in Uniat Test hall fo. 2 and 1,434 foet In Uhate.t Test liell No. 1 cuntuin 69-71 percent garizet, 7-9 porcent zircon (length to width ratso, 481), 7-9 porcent brom tourralino; aid 1-2 por-

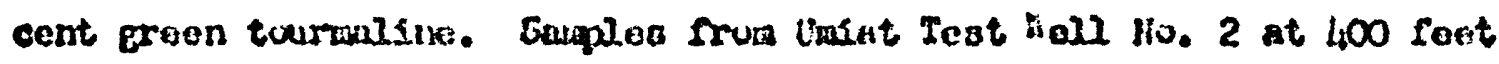
and from Uniat Test "ell Ho. 2 at 1,350 fect contain 19-29 porcont garnot, 9-10 percent atrcon, and 30-45 percant tourmalina. This accurdance of amples is the basis for corralation of Orat Jost Hell Hoo. 2 and 2 11lustratod is sigure 5. 


\section{Correlation of faultod soction, traiat Tast Fells Ros. I and 2:-A}

reveroe fault has been recombed in Uad at Test Nells Nos. 1 and 2.

R. O. Reeso $L$ has coscribed tho fault as cutting biait Test fiall Ho. I

at 2,045 feet, wh a throw of 750 feet, and with the secuion from 2,300

to 1,950 feet being repented in the scction from 2,045 to 2,700 feet. To

cubstantiato this corrolation coparisons of heavy-inaral oultes rero wido. In rigure 5 and also in taje 3 the section balow the failt 13 shom opsosito its supinsorly squivalent section. On tho basis of the avallable sapples the folluring houvy-rinural correlations aro apparent.

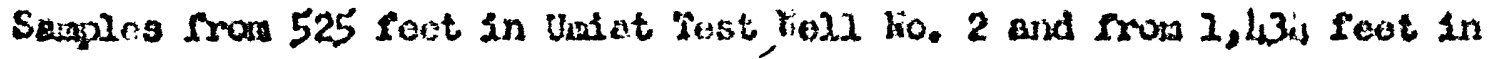
Owiat Pest Foll H. 2 mhich are placed in subaros $U-6$ apparently belch; to tho sano cubzona as the sarala frosi $2,270^{\circ}$ foot in tho prujected fault

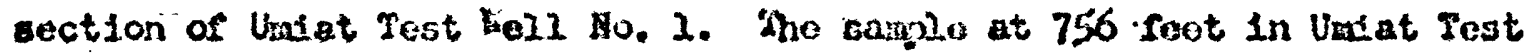
Tell No. 2 which is placed in oubuono U-5 becias to corralete with tho aanplo from 2,337 foat in tho subfaut aoction of Valat jest fiell No. 1. Tha subsone U-3 saigle frua 1,792 feot in batat lost Mell ko. 2 seeso to

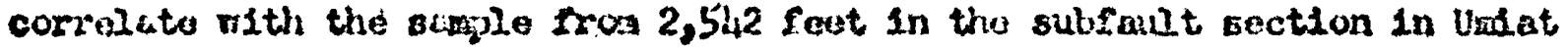

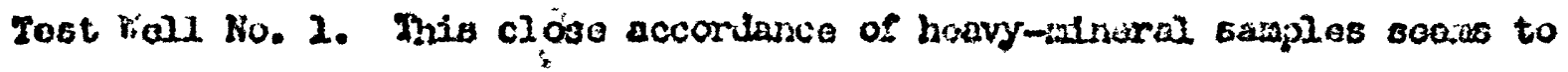
oubstantiato tho intorprotation of tho rault in liadat res: Moll No. 1. Corrolations of Untat Iest Malls fos. I and 2 and of the faulted section

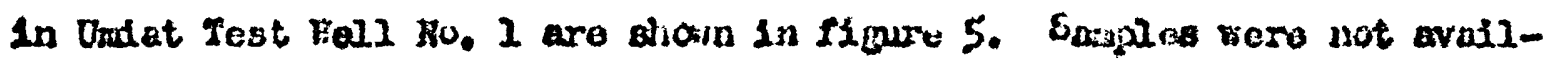
ablo to chock tho fault In Ondat reat fiarl $H_{0} .2$. 


\section{IHERPRETATION OF WEAVY KTKRALS OF SEMTLUTASX ROCKS OF}

WORTHEH NLASG, IN EFLUTION TO THE OFOSOIIC HISTORY

Triastefc

The irfasste sodirantisy rocks of northern Alaska are represenced by an wluost untforis thleknuss of alightiy less than 300 foot of black shale, silty sandstoro lenses, 2trasstork and chort. These rocks are inom in the

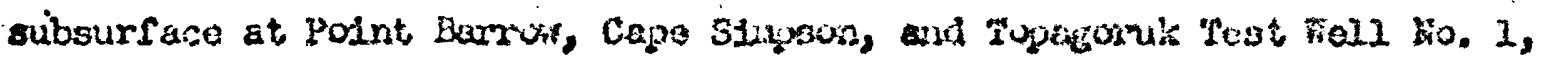
and in outcrops in discontimious exposures froa lapo liaburno to the sac-

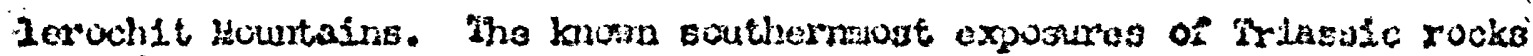

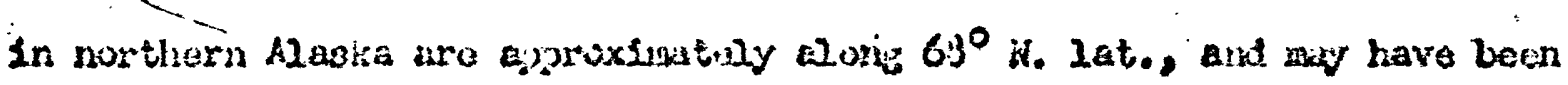

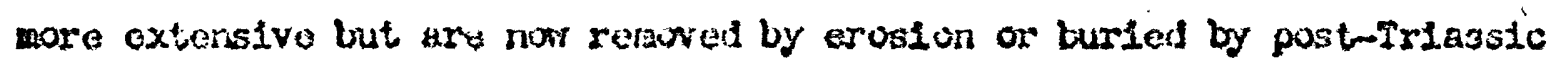
thrust fawte. In tho Upper Triasaic (Noric) degosits, tho peisecypod, Lunosis aubcircularis (aabb) io the nost abundant fussil but is nct roliable es a criterion for futarproting the dajth or the sea bottoa. On the

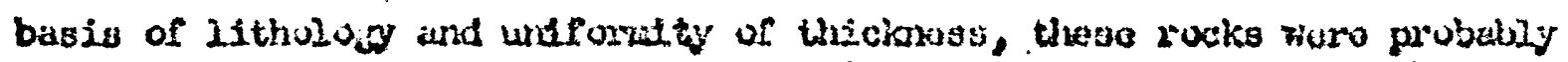

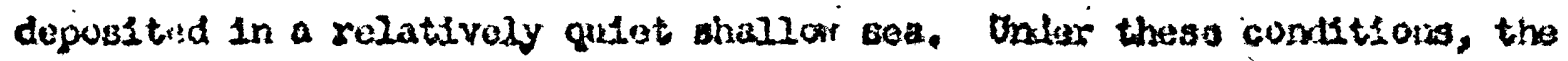
major agerits of aedintant trinsportation and distribution wouid be wave action and bottoia curraris. The net effect of sucin activity rouid bo the eventual destruction of 2035 rosistant alnoril grains and abraglon of the

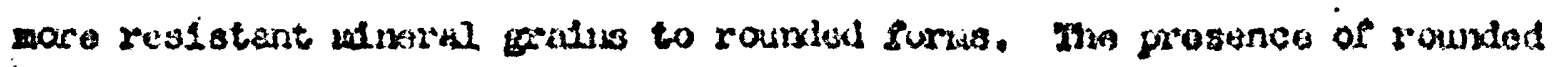

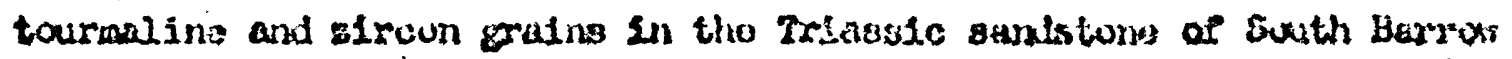
Teat holl No. 3 may bo attributad tu tho above-santionad processes. The Triass1c doposits raty ov $n$ bo conyosed of grains that had alroady gono through at lowst one cyclo of doposition and wero rounded prior to Iriassic time. 
Thus far, littlo ovidanco regarding the Bource of Trisssic rucks has boon found. Onforinatoly factes pattoms of knorn Iriassic rocks, in northern Alaska, do not Indleato the probabio soures area or how far the graina have travaled. One possibio sourve rock, the Heruokguik schist of possible pro-Candrian azo, crops out 30 whes suth of tha Eubleroshit

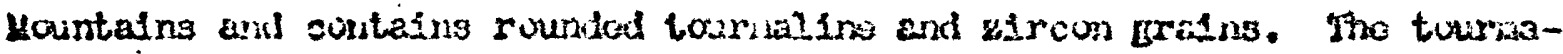

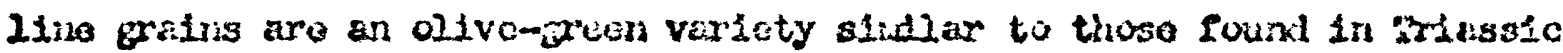
Eandstune et point berron. Whether or not the lredriokguk ochist wes exposed to erosion here or elserhere during mingsic tisto is not knum; carly Cretaceous axd yun or poriuds of faulting have brought it to its prosent postition at the surface. The sind larity of tur talino greins in tho Neruokpuk schist and the Triassie andst me at Point Barror is the only

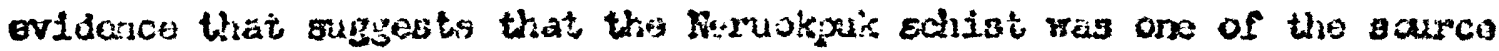
rosks of Irlassic dujouits. Potrolois of other promriassic rucks which ahould also bo considorat as possiblo ources is too inadequataly kncarn to justify discusplon.

Jurassic

In the Point barrui-Capo Simpon aruis Nurassic rucks rest conforiatily on Triassic; doposition apparentiy wes continuous. In tho sụthorn brooks

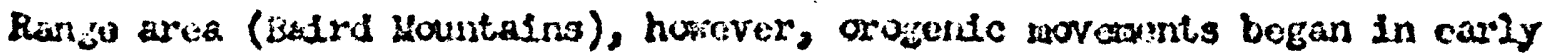
Jurassin time. Initlal east-trending thrust faults and folds were devoluped as a result of coragrussional forces Iran the Bcuth. Rocks Involvisd in this

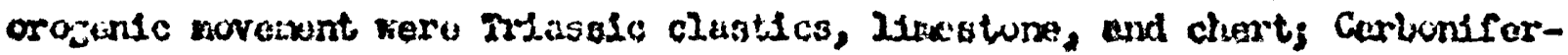
ous-Perwan 21uxeatona, chort, aud alnstics, Davonian clustics and 21mostono; and slurian and pro-silurian preonstons alist, wea schiot, chlorite ech1st, quartsito, calcarovis wchiot, and carbunaceous schist. rarle 
Igneous Intrualves and extrugives wore eaglaced and the orea becase a landwess. During this time the shoreline regresged slonily northitard but prou bably nover extended auch farther nurth then 2 at. $68^{\circ}$. Nlong the eouthorn 2ittoral and noritic zores, vurassic beilinents wero depositod in ansulax relationships with Trlassic and older rocks. That this orogen, with its developsent of a Ifthologically coujlex source area, is recorded in the

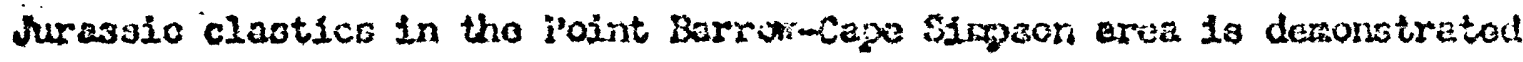

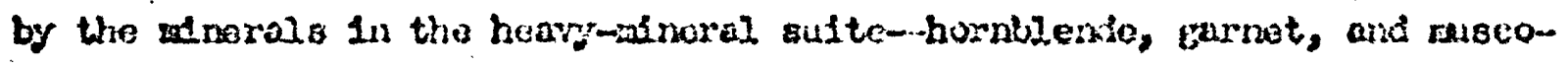

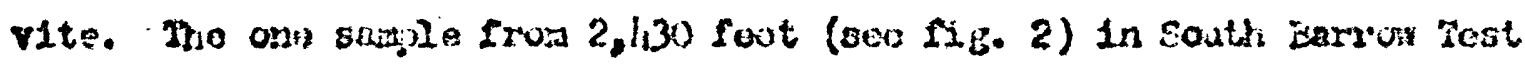

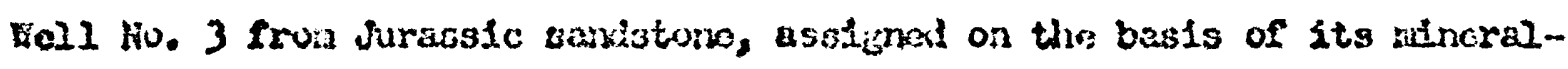
ogic suite and grain shaye, to the rounded tourwalino gork, laplies that tho earliur part of Jurazic solinantation in tho Burrow orea was eliatlar to Triasuic deposition. The sandstones higner in the surazsic ouction, as

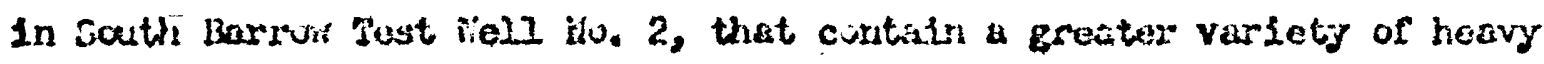
minerals rufloct the delayed, though nowat, changes in the sounce area. Though the grains probably travoled a Inneur diatance of apjrosdiately 300 miles they wor not subjocted to as sutanco osciljatory action or rosoricing as varo tho grains frua oldor Jurussic and irlasistc sandstone, as Bhorn by the prisuatic tourzal fno grulna.

\section{Crotacedus}

In the aren of tho present Irvols Rane exother orogeny that foldad

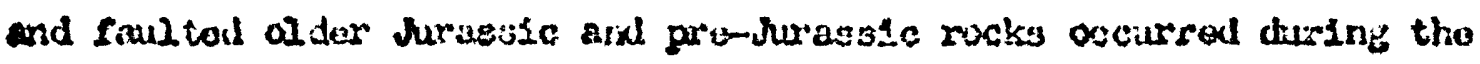
Interval betrena Late Jurasaic and Rarly Crataceous. Nafic intrubions

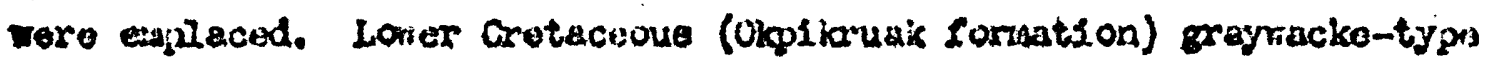
clastics wero depoested unconformatily on this structural couplox. North- 


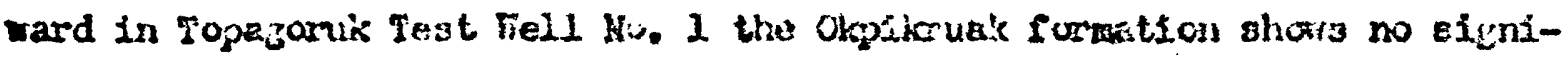

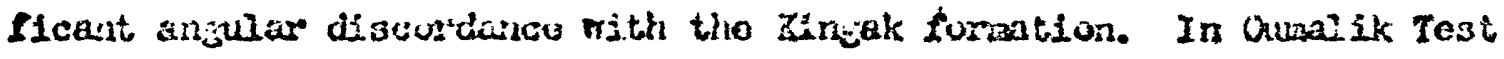

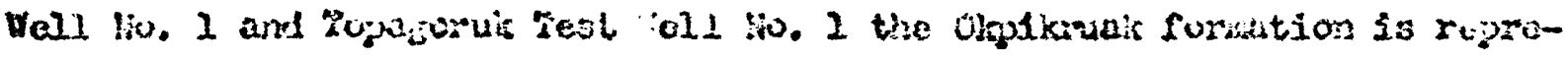

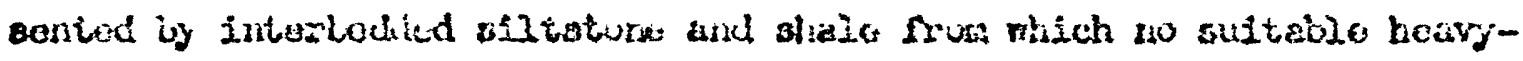
afrerai cuscontrubes culd bo propared.

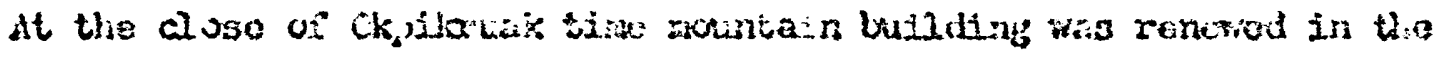

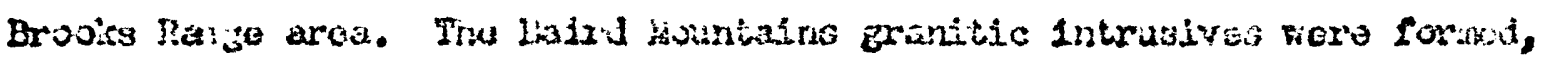

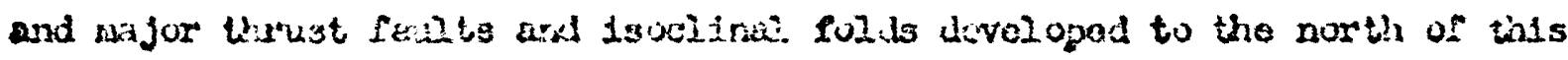

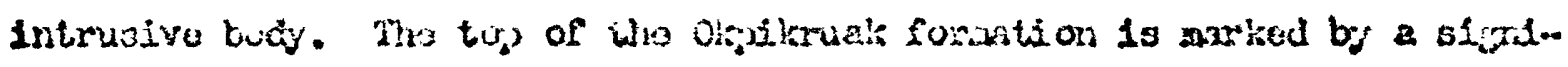

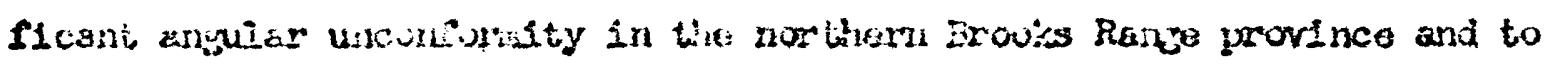

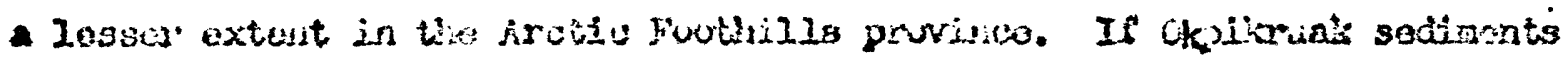
were dojosited in the Bixsur ared, thoy wore probably thin and wero eroded during this trix, infurrin: tive positive satire of the bar rois high. The

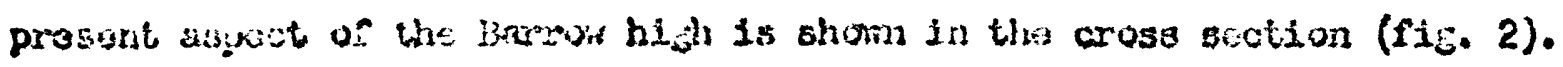

Tho Torot formition, a typleal breyracts, was deposited on this inegular suráace. Ahe Toruc furation consists of a Bouthern fectes of

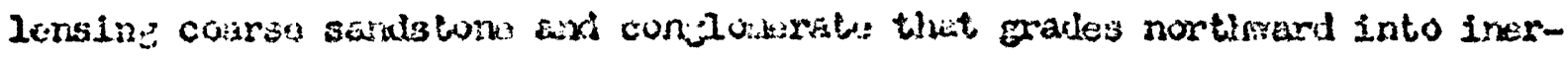

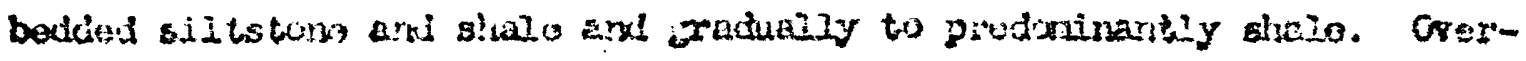

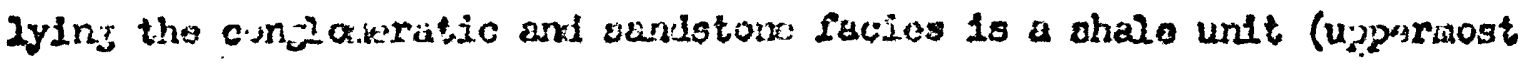
Torok) that represonts a sunthrarl wisiston of the soa durine upive forok thise.

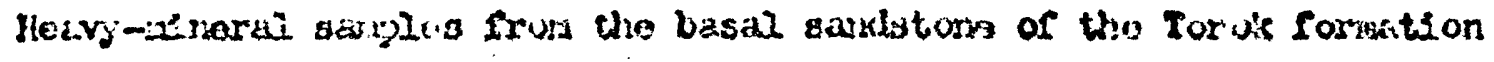

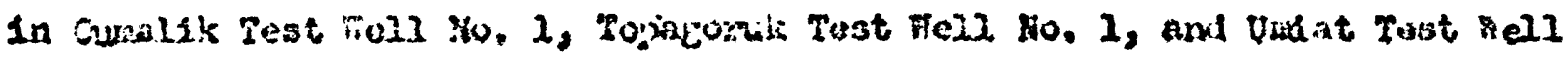

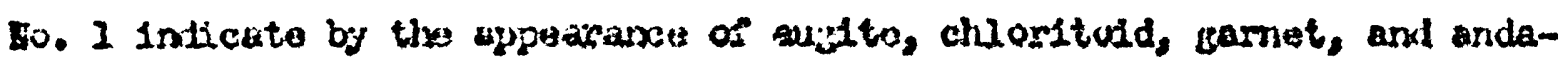
luslte, that hirassic miflo igreous rucko and earlien motamorghio rocks were cupused to arveion curlni early Torok time. Ls croalon proceadod in 
tho southarn Brooks Rango arod, the sea of late Forot thso trenserossed farther and farther south end covered at 2cast moch of the oroa now repre-

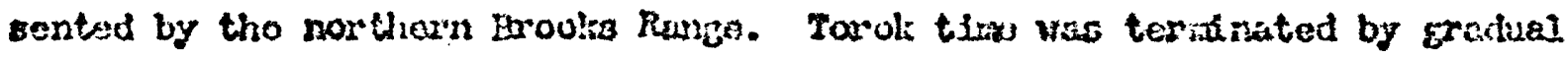

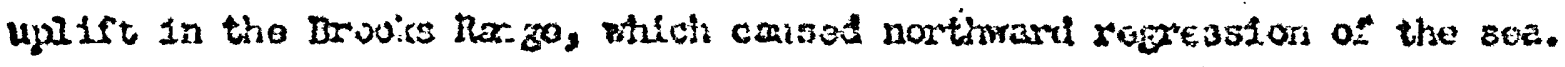

Inis rouresoion ingtieted tanushis goup doposition in tho northern

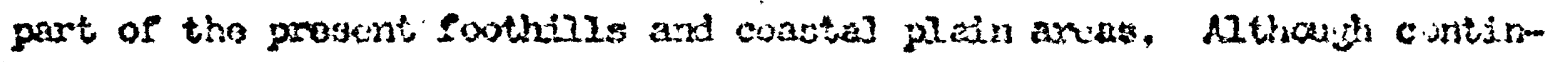
ental facios of this zover part a the karubhuls groug exo injuin to the

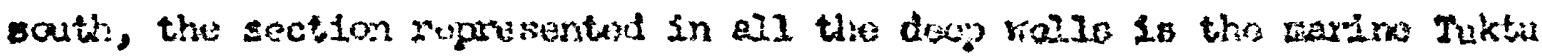

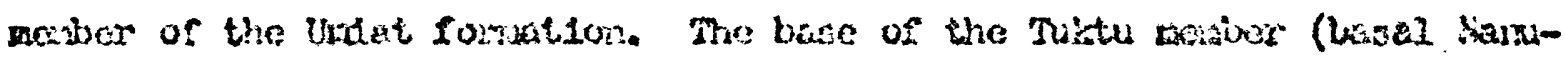

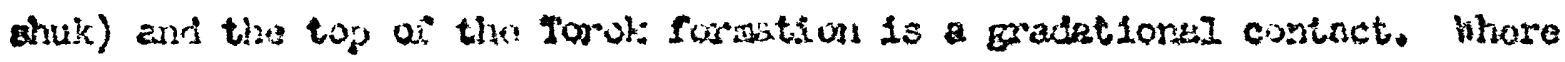

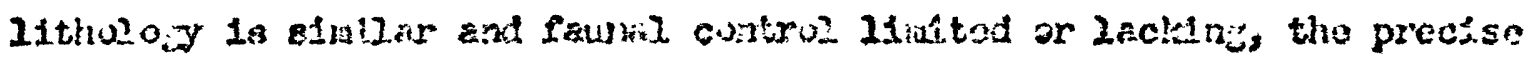

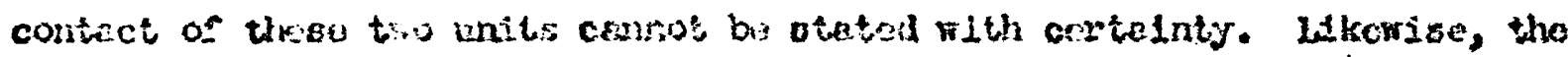

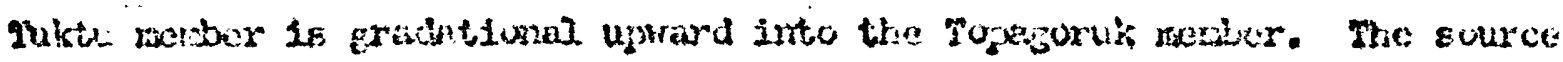

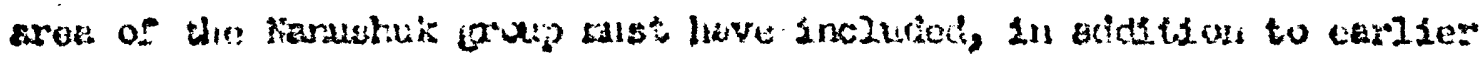

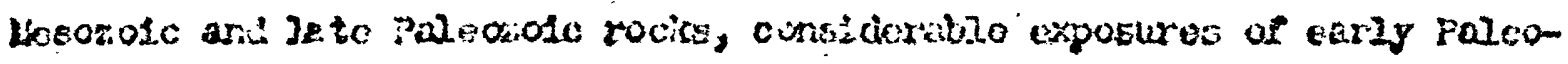

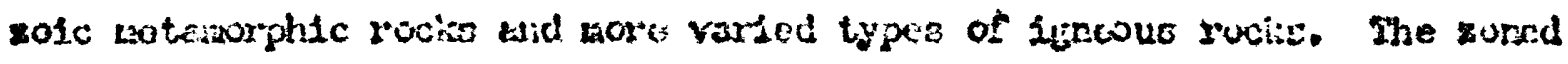

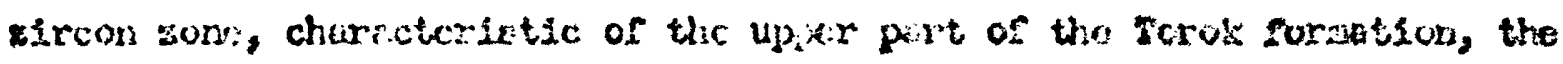

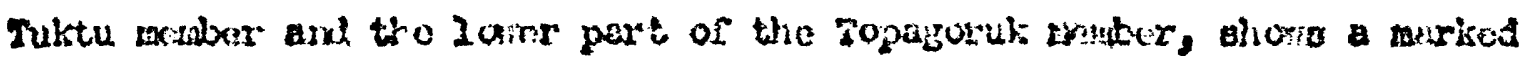
Incrosso in tho occurrence of chioritosd, kndizueite, end garnot-mincrals cheracteristic of dynantcaly wotenorghosud rocks, Plcotito wey have bean

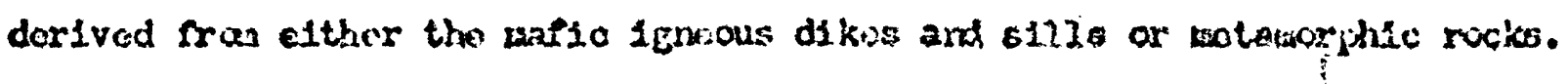
The tulitialine graine thet have oruld cores survumled by overerorths with fresh cryetel faces suggost derivation fruss uld eandstones and notrinorpliosed sedinctitory rocks that had been mireraliwed. De euhadrai tourwalino.

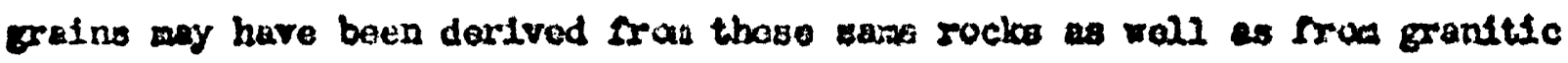
Intrusives in the Baird Lountalns. The soned sircon eryetals were probebly 


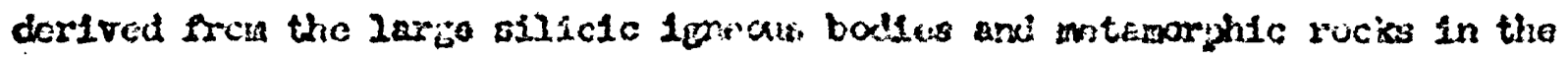
Baind lountidns. The influx of these various whrerals in the vper part

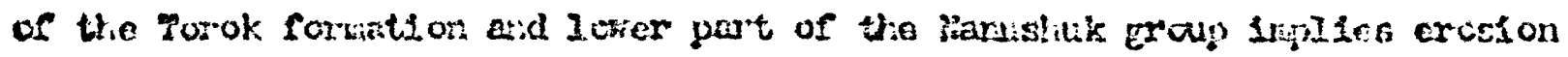

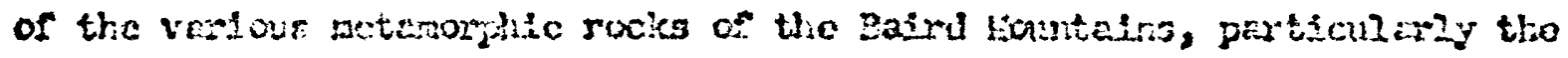

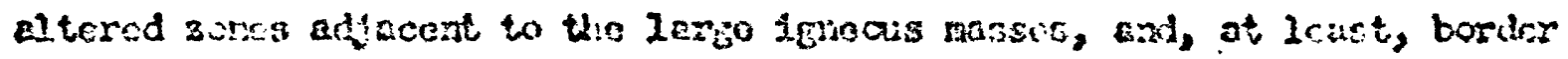

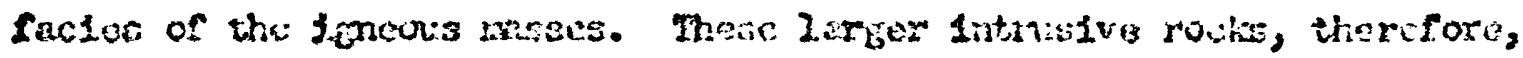

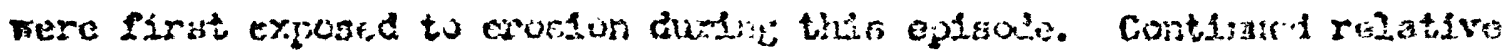

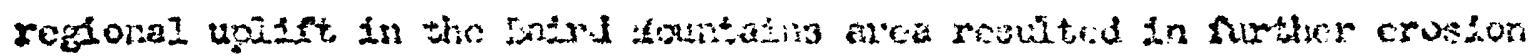

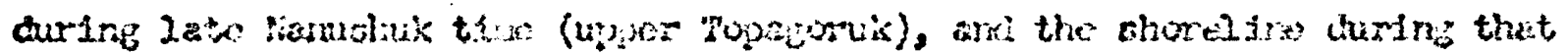

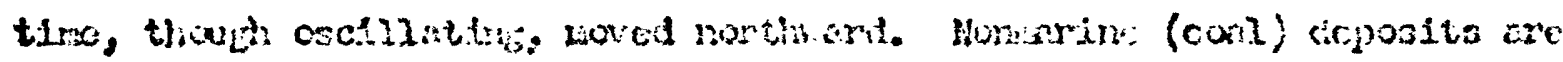

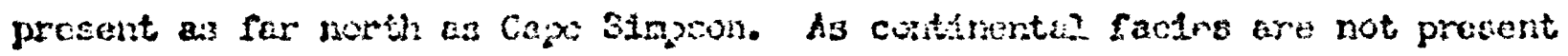

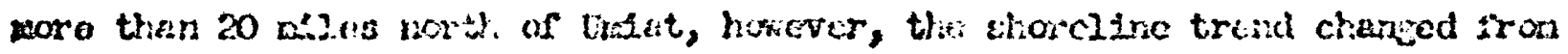

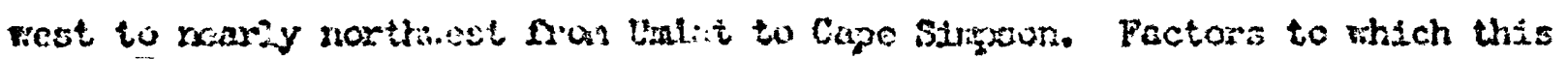

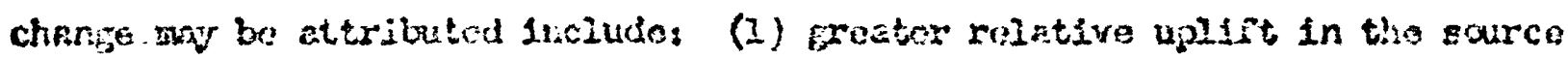
aria fiest of luat thon in the castern aroa; (2) creater thiclabeces of deposits and cunsegurnt fulling in of the vestern part of the troudi; (3) depuition of crotaceaus eediarnts in besing sauts and north of the

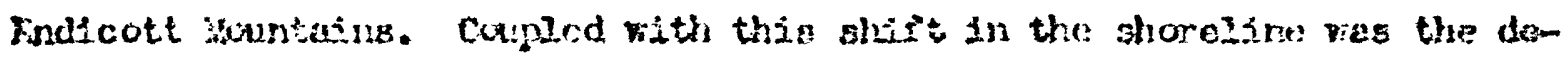
velogazent of tyo veatrontary putrograghe provinces in upper Tonazoruk

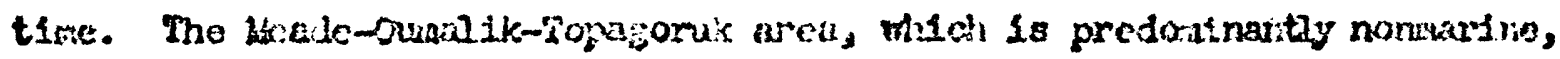
16 reprosented by the euhctial asrcun zone, and the Ungatmish Creek-

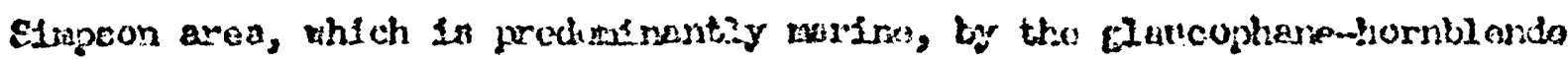
rono. Although the geugraghic distribution of these zines is prastically

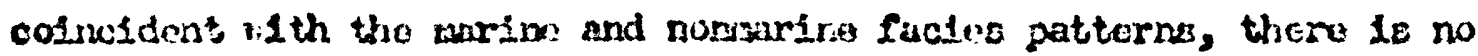
Implication that they arn 80 Ifrutad; the plaucophanofiornblendo ione occurs In nunwarine and warimo dopoosto where thos intertonour. Of groat sicni- 


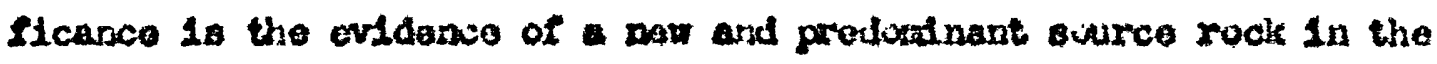
eastern part of the Erooke Range as thown by the occurrence of glaucophano and the "ulue-preen" bornblemle. Drectly south of Jatat in the

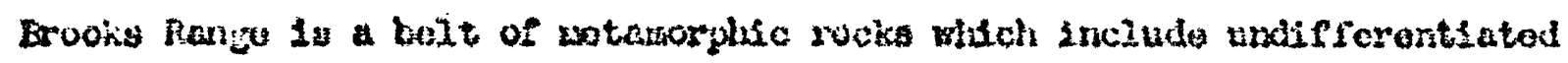
silurian and avortan and alder schist3. In the 1951 field season this area was studied by a Gojogical survo field purty. Tho observatsons of

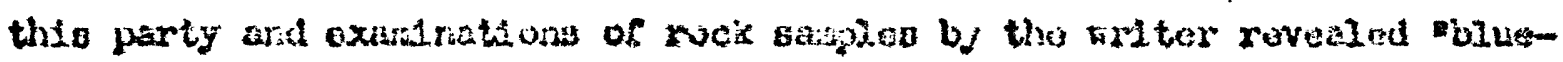

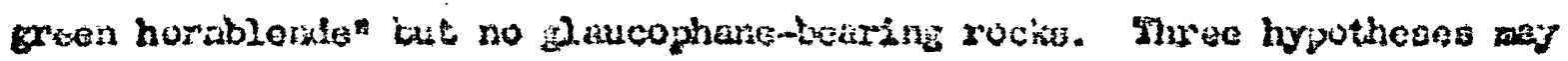
explatn the absence of ench ruoks at the prosent gurtack (1) the gleucophane rocks havo been curglately resuved by eruston; (2) the elaucophare rocks bevo not yet boen lientified in the browita nanke; (3) the glanco phase rocics bive been turiad by overuhust sheots of different idthologic character during post-rupazoruk tisa, Of thesa, the second is the aust 21kejy explanstion, Hithout this eritical inforation only brasd genoraliations can bo stated regarding the scurce rocks of the blaveuphasehornblonio zoreg that is, that the source aroa lies within the osstera

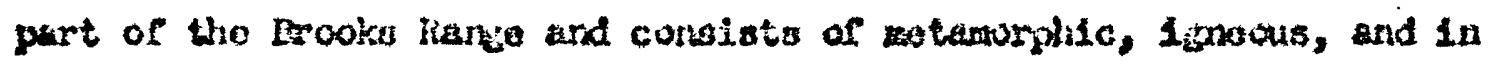
part ulder selinsontary rooks.

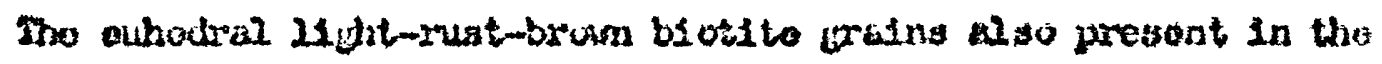

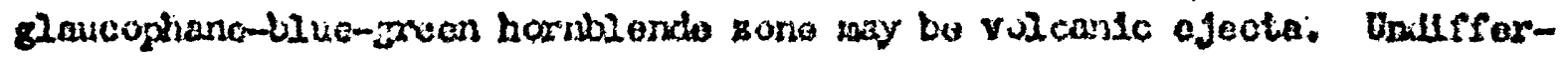
entiated kesozole volcanica are knom in the cowars Pentrusula and in the

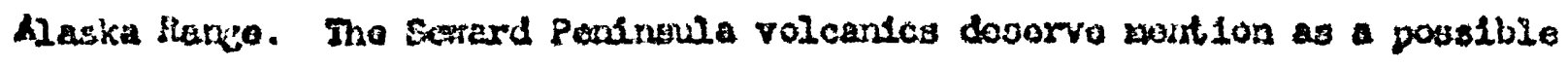

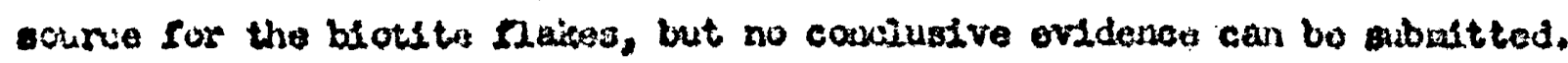

In tho westorn aroe (Meado-Oudallk-Fopngoruk Rivere area) sandstones of the uppor part of the jopagonuk womer aro charactersued by ainarals

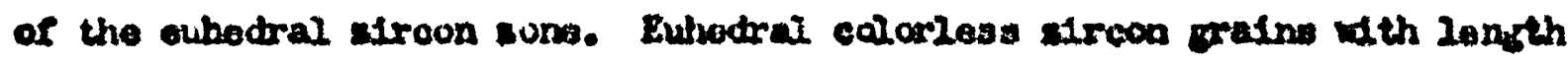




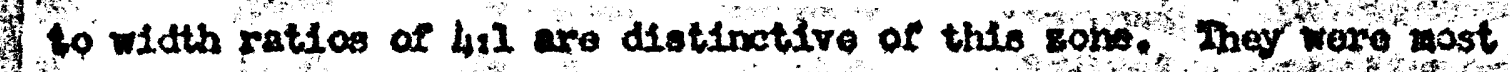
probably derived frut tho Batrd bavintain grantilo intruolvo and frota creater doptho withen this boty than the goned afrcon prains of tho soned sircon aune. Doeper crosion of the crantic nas 10 therefore Iaplial during lato Topagoruk time.

Defferential uplift with the Ereatest elovation in the area nost of

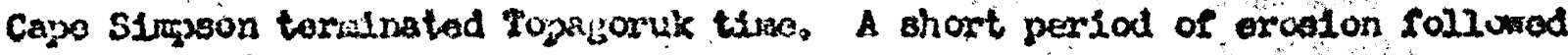
after which tho area was subserged. The encroaching sea estended ahurt distance south of Undat but probably did not cover Posnt Barrow or the wostTern part of Cape Sinpson. Colvilia moup ocdinents waro depostted north and northoast of Unat, Ihe seabeo mabar, oldest part of the Echrader Bluff fomation, rasts unconformably on tho Topagoruk abubar and is canposcd of black marine shalo with some andy boutonttc bads noar the baso. Sanjles froi those bontontito bods contain an abunilance af blotite grains and are therafore tarad tho biotito rone. The blotito grains are lightrust and dark-brom euhetra. Because of thair intirite assoctatson with

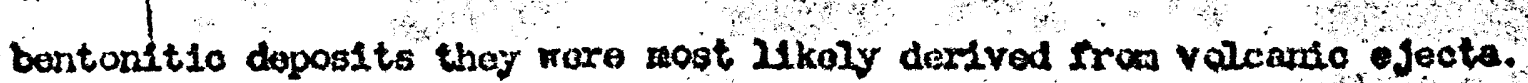
The undiffuruntiatad ussozolc volcanics of tho samard Peninsula are, again. the only knom volcanoas which could have ouppliod tho bontoritio naterial. As theso volcanoes are bout 1,00 niles distant and proof of their activity durline this exact tise is lecking, a reagonable dubt wist bo malitainod If thay are to bo cunstderad as a source.

If erosion of pre-Colville group rovios is postulated as contributing the blott to grains, the only posalbie scurce would be the uppor part of t the ropagorik momber. Blotito praino of aldar (pro-uppor part of topagoruk)

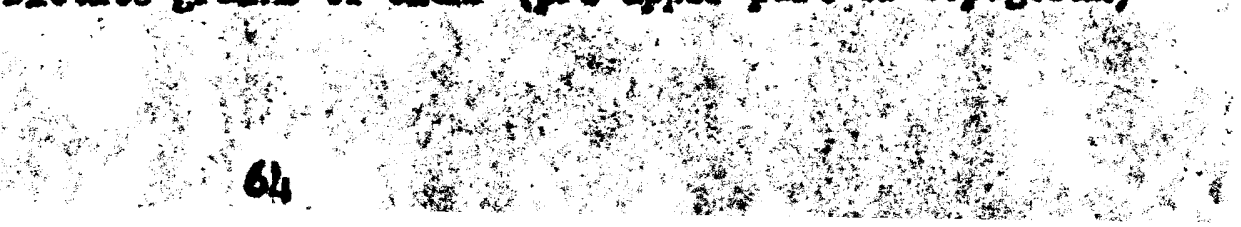


rocks aro scarce and not of Identicul varloty. Biotite prains in tho upper part of tho Topagoruk menber are not as abundant as in tho seabee mober, boine 2 ess then 50 porcent of the heavy-nineral suite, wisin in the seabou mbor thay compose over 90 percent of the heary-joneral ousto. Concentration by sedimentary processes is highly unlskely. A volcanic origin is nore acceptatie, even thatgh a dofinsto scurce area cannot be desionstrited.

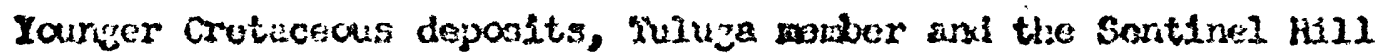

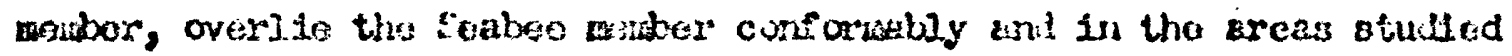
aro praduinentiy thale. At the close of the Cretaceous poriod tho frctic Slopo was unjirted. Inrilary continenial deposits aro rostrictod to the area cast of the colville Iigver nurth of urat aml north and vest of the

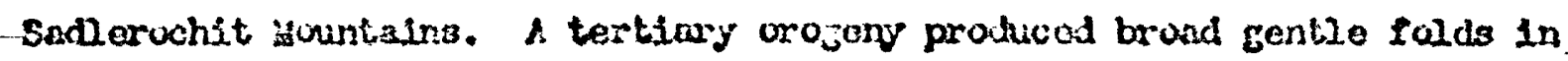
tho Arctic Foothills and Nritic Coastal Plain provinees. In part, these folds waro supariaposed on oldar structures.

During the Plolotocine epoch of the Quaternary period glaciors occuplod the Erooks Ranio. Outriakh gravols fllled valloys Blong the wountain Erunt and nost of the 1 oxlauls of tho coastel plain aroa. Lockily marino ohellor-water deposits are presont. In the coastal plain aroa these marino and nomarino clastics, with sopo possiblo kecent dejosits aro tho Gubik formation. Thoso deposits reru darived fros all older rocks. Hovorkinis processes wore vigorous and prains aro rail-prounclad. Tho heavy-mineral oulte of the cubik formation includes ruundod grains of all aineril rarleties and specius found in the oldar rocks. 


\section{BUthapt}

Hescy sinoral reslues Irus sudstonos of test nolls and core

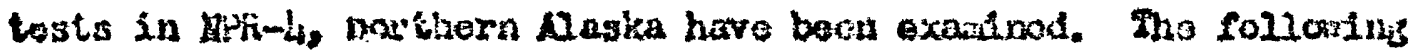

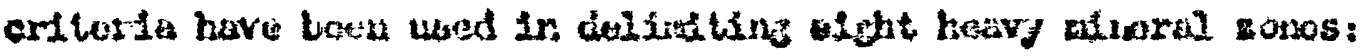
(1) pressnce of dixpnostio marals or minural oultes; (2) relativo

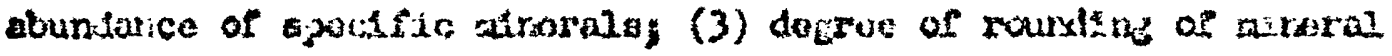

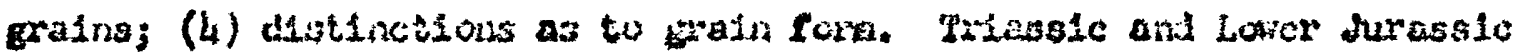

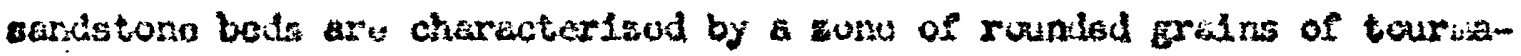

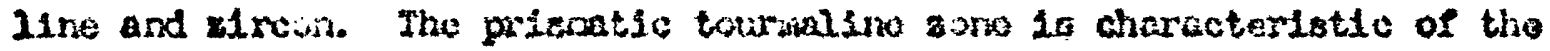
upior part of tho Jurassic in the sarron arsa. In the crotacous codimentary rocks tho augitamajoconte suna 18 rapresentativo of the luwer part of the serois foruation; tho zonad eircoin zono is represontative of

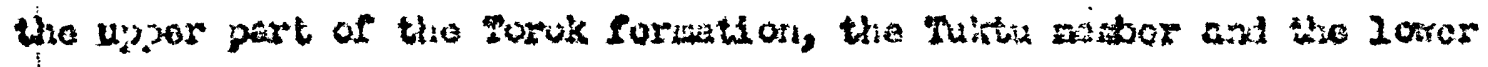

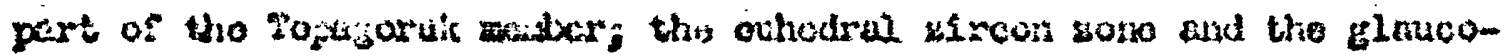
phana-b2uo gruau hornblende son aro reprasontativo of the upger part

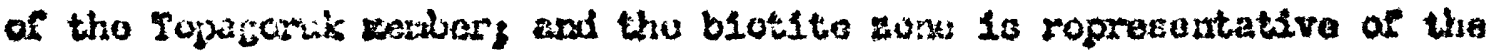

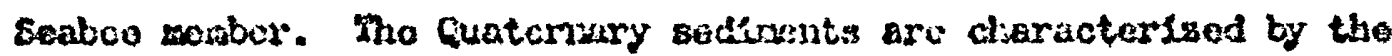
roinded grain zoms.

Sowo of those ronss are known only in $20 \mathrm{cnl}$ aroas, others havo been

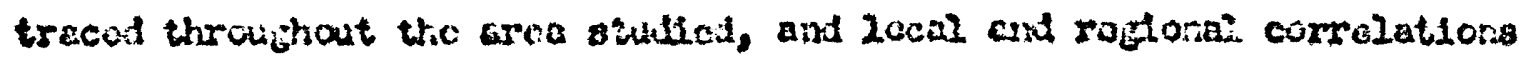

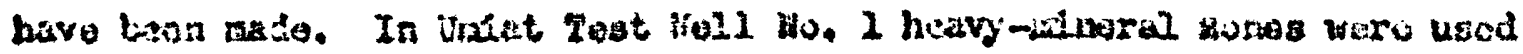
to correlute a auction ropsated by feulting.

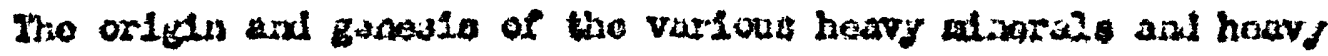
unoral sulter have boen. Intorproted in ralation to goalogle hietory. 


\section{BTHLIOCRAPIY}

Dana, Fidwerd 3., A taxtbook of minuralozy: Ed. Filliai E. Road, John Villoy \& Sons, Mow Iork, 1947.

Orout, Prank F., Petrography and petrology: LaGizair Hill Buok cunpary, Now York, 2932.

Xrubein, F. C., and Pettijohn, E. J., Wamial of sadiwontary potrography: Ajpinton-Contury-Crofts, Inc, l lew Ior's, 1933.

Srynino, Paul D., Petrology and gamals of tho thisd Exadrord sand: Pensylvania State College, Ilimeral Industrles Bxporisontil Station Iuli. 29, Ponnsylvania Stato College, Pounsylvania, 2940 .

Lertio, J. B., Jr., Geolosy are! goid plecurs of tho Chimialar Dtetrict, Hinevul Fosourcos of Alaska: U. S. Ceol. survey ball $773,1923$.

Lountain bullding in Alaske: An. Jour. Sc1. rol. 20, Ev. $116,2930$.

and Sinith, P. S., Oeology and minoral requurces of nortimestarn Alaska, U. 3. Cicol. Survoj Bull. B15, 2930.

Hiner, H. B., Sodimentary patrojrajty: burb, and Compeny, Iomion, England, 2d. odi., 1929.

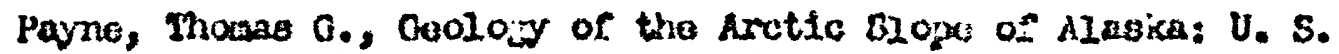
Gevi. Surray Oil and cas Imestigations yap 0id 126, 2951.

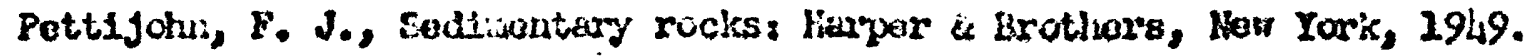

Hugers, A. F. and Eorr, P. F., Thin section mineralogy. LicGraw Hill Book Coranany, fien Iorli, 2933.

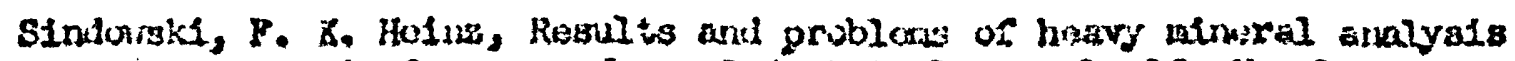
in Cerming, Jour. Sod. Potrolo:y vol. 29, H. 1 , April 1949.

Sidth, Philip S., Arvil geology of Alarica: v. S. Deol. Survey Pror. Papar 192, 2939.

Kobuk-Loatak reifion, Mlaska; U. S. Geal. Survey Bull. 536. 2913. 
Iickell, Trederick G., The exarination of Nragantal rucksi stanford Univ. Press, Stanfurd Univ., Californta, 2931.

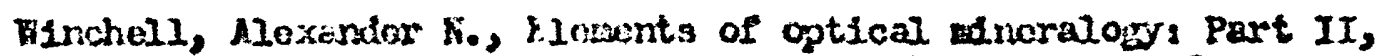
Description of tanerals: Joln tilley end Sons, Inc., Nen Yurk, 1933. 